Acta Math., 219 (2017), 65-134

DOI: 10.4310/ACTA.2017.v219.n1.a4

(C) 2017 by Institut Mittag-Leffler. All rights reserved

\title{
Singular Ricci flows I
}

by

\section{Bruce KLEINER}

Courant Institute of Mathematical Sciences New York, NY, U.S.A.
JOHN LOTT

University of California at Berkeley Berkeley, CA, U.S.A.

\section{Contents}

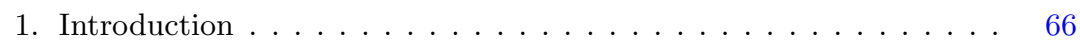

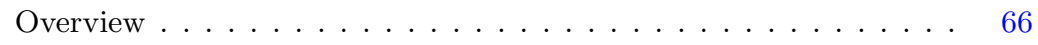

Convergence of Ricci flows with surgery . . . . . . . . . . . . . . . 68

The structure of singular Ricci flows . . . . . . . . . . . . . . . . . . 72

Related work . . . . . . . . . . . . . . . . . . . . . 75

Concluding remarks . . . . . . . . . . . . . . . . . . . 75

Organization of the paper . . . . . . . . . . . . . . . 76

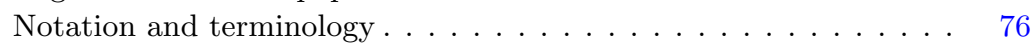

Part I . . . . . . . . . . . . . . . . . . . . . . 77

2. Compactness for spaces of locally controlled geometries . . . . . . 77

2.1. Compactness of the space of locally controlled Riemannian man-

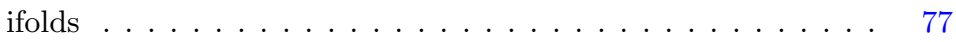

2.2. Some properties of controlled Riemannian manifolds . . . . . 79

2.3. Proof of Theorem $2.4 \ldots \ldots \ldots$. . . . . . . . . . 80

2.4. Compactness of the space of locally controlled spacetimes . . . 81

3. Properties of Ricci flows with surgery . . . . . . . . . . . 83

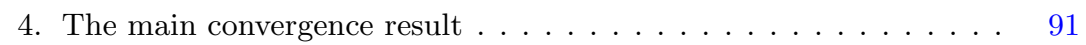

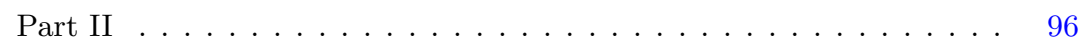

5. Basic properties of singular Ricci flows . . . . . . . . . . . . . . 96

5.1. Maximum principle and scalar curvature . . . . . . . . . . 96

5.2. Volume . . . . . . . . . . . . . . . . . . . . . 97

5.3. Basic structural properties of singular Ricci flows . . . . . . 100

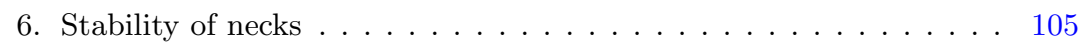

6.1. The main stability asssertion . . . . . . . . . . 106

6.2. Convergence of $l$-functions, asymptotic $l$-functions and asymptotic solitons . . . . . . . . . . . . . . . 106

6.3. Stability of cylinders with moving basepoint . . . . . . . . . 109

7. Finiteness of points with bad worldlines . . . . . . . . . . 111

Research supported by NSF grants DMS-1105656, DMS-1207654 and DMS-1405899, and a Simons Fellowship 


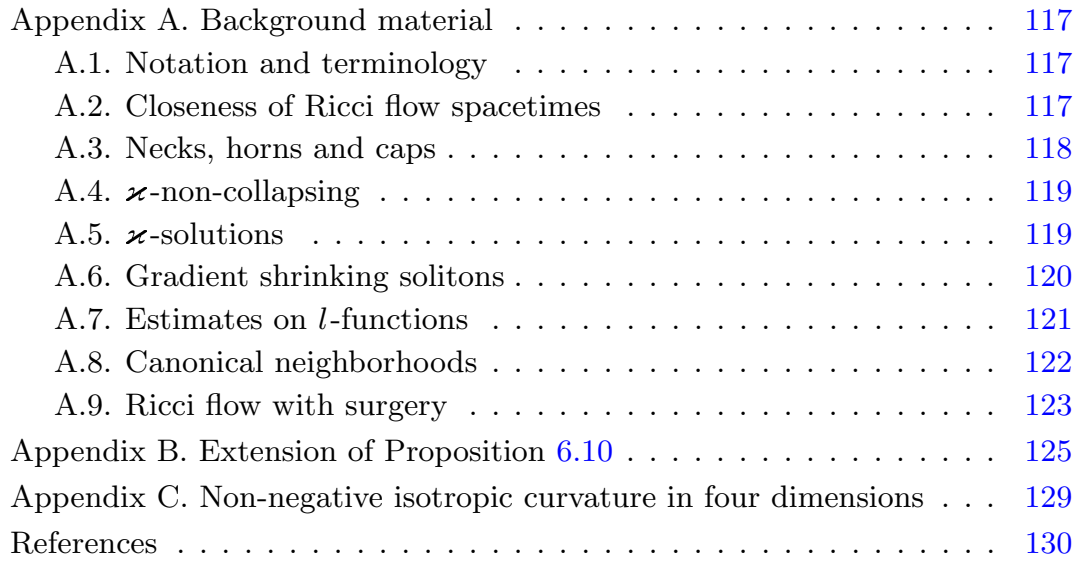

\section{Introduction}

\section{Overview}

It has been a long-standing problem in geometric analysis to find a good notion of a Ricci flow through singularities [30], [57]. The motivation comes from the fact that a Ricci flow with a smooth initial condition can develop singularities without blowing up everywhere; hence, one would like to continue the flow beyond the singular time. From the broader perspective of the analysis of partial differential equations (PDEs), this is just one instance of the widespread phenomenon of the breakdown of classical solutions, a phenomenon that is central in geometric PDEs, and which is often handled by using generalized solutions. For example, for mean-curvature flow of hypersurfaces in $\mathbb{R}^{n}$, there are notions of generalized solutions [9], [15], [25] which became the foundation for studying existence, uniqueness, partial regularity, compactness, and other structural properties of solutions [9], [26], [42], [43], [78], [79]. Other geometric PDEs, such as minimal surfaces [1], [2], [23], [28], [29], [60], [61], [65], [70], harmonic maps [8], [31], [40], [48], [55], [63], [64], [66], and harmonic map heat flows [16], [49], [72], have undergone a similar development.

Thus far, there has been little progress in implementing a similar program for Ricci flow. In the approaches used for the equations above, one first defines "rough" objectse.g. integral currents, sets of finite perimeter, varifolds, Sobolev mappings - and then, using an appropriate approximation scheme, one produces a generalized solution within the class of rough objects, by appealing to a suitable weak compactness result. Certain features, such as the existence of an ambient space, or the fact that one has a scalar 
equation, help enormously. The absence of these features in the case of Ricci flow creates serious technical obstacles to using such an approach with Ricci flow.

In this paper, we solve the problem of flowing through singularities in 3-dimensional Ricci flow. We do this using a novel approach that is quite different in spirit from earlier work, and which may be adaptable to other geometric PDEs. We introduce generalized solutions that we call singular Ricci flows. They are smooth Ricci flow spacetimes that are possibly incomplete, but are subject to certain asymptotic conditions. We show that singular Ricci flows have a number of good properties. In particular, we prove a compactness result for families of spacetimes, and using this we obtain the following existence theorem.

THEOREM 1.1. For every compact Riemannian 3-manifold $M$, there is a singular Ricci flow with initial condition $M$.

In addition, we establish a number of structural results. These results, and further results that will appear elsewhere, strongly indicate that singular Ricci flows provide a natural analytical framework for 3-dimensional Ricci flows with singularities.

The notion of singular Ricci flows derives partly from the spectacular work of Hamilton [35] and Perelman [58] on Ricci flow with surgery. After constructing Ricci flow with surgery, Perelman was naturally lead back to the problem of flowing through singularities. In [57, §13.2], Perelman wrote: "It is likely that by passing to the limit in this construction one would get a canonically defined Ricci flow through singularities, but at the moment I don't have a proof of that". In proving Theorem 1.1, we partially confirm Perelman's expectation, by showing that Ricci flow with surgery (for a fixed initial condition) subconverges to a singular Ricci flow as the surgery parameter goes to zero. $\left({ }^{1}\right)$

Several aspects of the work in this paper may be applicable in other settings. First, the strategy that we use here-defining generalized solutions using smooth (possibly incomplete) spacetimes satisfying asymptotic geometric bounds - may be adaptable to other geometric PDEs. In addition, there are technical ingredients in our work which may have parallels in other situations, such as a compactness theorem valid for possibly incomplete Riemannian manifolds, and a dynamical analysis of ancient solutions. A novel feature of the setup is that the spacetimes are not assumed to be simply concatenations of product spacetime regions. To our knowledge, this is the first appearance of such

( $\left.{ }^{1}\right)$ Note added in proof. Since this paper was submitted, there have been several developments building on the results proven here. Perelman's convergence conjecture ([57, §13.2], [58, p. 1]) and the uniqueness question (Question 1.6) were both addressed in [7]. These results, together with a number of results from this paper (the existence of singular Ricci flows (Corollary 1.5), behavior of volume (Theorem $1.3(4)$, Theorem 5.5), and finiteness of bad worldlines (Theorem 7.1) were used to prove the generalized Smale conjecture concerning the homotopy type of diffeomorphism groups of 3-manifolds [6]. 
spacetimes in Ricci flow, although of course they are widely used in general relativity and mean-curvature flow.

We mention that there is related work in the literature on Ricci flow through singularities in certain cases, such as in the Kähler case or under the assumption of rotational symmetry; see the end of the introduction for a discussion.

\section{Convergence of Ricci flows with surgery}

Before formulating our first result, we briefly recall Perelman's version of Ricci flow with surgery (which followed earlier work of Hamilton). (To make this paper accessible to a wider audience, in $\S$ A.9 we collect needed background results about Ricci flow and Ricci flow with surgery.)

Ricci flow with surgery evolves a Riemannian 3-manifold by alternating between two processes: flowing by ordinary Ricci flow until the metric goes singular, and modifying the resulting limit by surgery, so as to produce a compact smooth Riemannian manifold that serves as a new initial condition for Ricci flow. The construction is regulated by a global parameter $\varepsilon>0$, as well as decreasing parameter functions $r, \delta, \varkappa:[0, \infty) \rightarrow(0, \infty)$, which play the following roles:

- The scale at which surgery occurs is bounded above in terms of $\delta$. In particular, surgery at time $t$ is performed by cutting along necks whose scale tends to zero as $\delta(t)$ goes to zero.

- The function $r$ defines the canonical neighborhood scale: at time $t$, near any point with scalar curvature at least $r(t)^{-2}$, the flow is (modulo parabolic rescaling) approximated to within error $\varepsilon$ by either a $\varkappa$-solution (see $\S$ A.5) or a standard postsurgery model.

In Ricci flow with surgery, the initial conditions are assumed to be normalized, meaning that at each point $m$ in the initial time slice, the eigenvalues of the curvature operator $\operatorname{Rm}(m)$ are bounded by 1 in absolute value, and the volume of the unit ball $B(m, 1)$ is at least half the volume of the Euclidean unit ball. By rescaling, any compact Riemannian manifold can be normalized.

Perelman showed that, under certain constraints on the parameters, one can implement Ricci flow with surgery for any normalized initial condition. His constraints allow one to make $\delta$ as small as one wants. Hence, one can consider the behavior of Ricci flow with surgery, for a fixed initial condition, as $\delta$ goes to zero.

In order to formulate our convergence theorem, we will use a spacetime framework. Unlike the case of general relativity, where one has a Lorentzian manifold, in our setting there is a natural foliation of spacetime by time slices, which carry Riemannian metrics. 
This is formalized in the following definition.

Definition 1.2. A Ricci flow spacetime is a tuple $\left(\mathcal{M}, \mathfrak{t}, \partial_{\mathfrak{t}}, g\right)$ where

- $\mathcal{M}$ is a smooth manifold-with-boundary;

- $\mathfrak{t}$ is the time function - a submersion $\mathfrak{t}: \mathcal{M} \rightarrow I$ where $I \subset \mathbb{R}$ is a time interval; we will usually take $I=[0, \infty)$;

- the boundary of $\mathcal{M}$, if it is non-empty, corresponds to the endpoint(s) of the time interval: $\partial \mathcal{M}=\mathfrak{t}^{-1}(\partial I)$;

- $\partial_{\mathfrak{t}}$ is the time vector field, which satisfies $\partial_{\mathfrak{t}} \mathfrak{t} \equiv 1$;

- $g$ is a smooth inner product on the spatial sub-bundle $\operatorname{ker}(d \mathfrak{t}) \subset T \mathcal{M}$, and $g$ defines a Ricci flow: $\mathcal{L}_{\partial_{\mathrm{t}}} g=-2 \operatorname{Ric}(g)$.

For $0 \leqslant a<b$, we write $\mathcal{M}_{a}=\mathfrak{t}^{-1}(a), \mathcal{M}_{[a, b]}=\mathfrak{t}^{-1}([a, b])$ and $\mathcal{M}_{\leqslant a}=\mathfrak{t}^{-1}([0, a])$. Henceforth, unless otherwise specified, when we refer to geometric quantities such as curvature, we will implicitly be referring to the metric on the time slices.

Note that, near any point $m \in \mathcal{M}$, a Ricci flow spacetime $\left(\mathcal{M}, \mathfrak{t}, \partial_{\mathfrak{t}}, g\right)$ reduces to a Ricci flow in the usual sense, because the time function $\mathfrak{t}$ will form part of a chart $(x, \mathfrak{t})$ near $m$ for which the coordinate vector field $\partial / \partial t$ coincides with $\partial_{\mathfrak{t}}$; then, one has $\partial g / \partial t=-2 \operatorname{Ric}(g)$. Also, there is a canonical Ricci flow spacetime associated with any Ricci flow with surgery (see $\S$ A.9); we will often conflate this Ricci flow spacetime with the Ricci flow with surgery.

Our first result partially answers the question of Perelman alluded to above, by formalizing the notion of convergence and obtaining subsequential limits.

THEOREM 1.3. Let $\left\{\mathcal{M}^{j}\right\}_{j=1}^{\infty}$ be a sequence of 3-dimensional Ricci flows with surgery (in the sense of Perelman), where

- the initial conditions $\left\{\mathcal{M}_{0}^{j}\right\}$ are compact normalized Riemannian manifolds that lie in a compact family in the smooth topology;

- if $\delta_{j}:[0, \infty) \rightarrow(0, \infty)$ denotes the Perelman surgery parameter for $\mathcal{M}^{j}$, then

$$
\lim _{j \rightarrow \infty} \delta_{j}(0)=0
$$

Then, after passing to a subsequence, there is a Ricci flow spacetime

$$
\left(\mathcal{M}^{\infty}, \mathfrak{t}_{\infty}, \partial_{t_{\infty}}, g_{\infty}\right)
$$

and a sequence of diffeomorphisms

$$
\left\{\mathcal{M}^{j} \supset U_{j} \stackrel{\Phi^{j}}{\longrightarrow} V_{j} \subset \mathcal{M}^{\infty}\right\}
$$

with the following properties:

(1) $U_{j} \subset \mathcal{M}^{j}$ and $V_{j} \subset \mathcal{M}^{\infty}$ are open subsets; 
(2) let $R_{j}$ and $R_{\infty}$ denote the scalar curvature on $\mathcal{M}^{j}$ and $\mathcal{M}^{\infty}$, respectively; given $\bar{t}<\infty$ and $\bar{R}<\infty$, if $j$ is sufficiently large then

$$
\begin{aligned}
& U_{j} \supset\left\{m_{j} \in \mathcal{M}^{j}: \mathfrak{t}_{j}\left(m_{j}\right) \leqslant \bar{t} \text { and } R_{j}\left(m_{j}\right) \leqslant \bar{R}\right\}, \\
& V_{j} \supset\left\{m_{\infty} \in \mathcal{M}^{\infty}: \mathfrak{t}_{\infty}\left(m_{\infty}\right) \leqslant \bar{t} \text { and } R_{\infty}\left(m_{\infty}\right) \leqslant \bar{R}\right\}
\end{aligned}
$$

(3) $\Phi^{j}$ is time preserving, and the sequences $\left\{\Phi_{*}^{j} \partial_{t_{j}}\right\}_{j=1}^{\infty}$ and $\left\{\Phi_{*}^{j} g_{j}\right\}_{j=1}^{\infty}$ converge smoothly on compact subsets of $\mathcal{M}^{\infty}$ to $\partial_{t_{\infty}}$ and $g_{\infty}$, respectively;

(4) $\Phi^{j}$ is asymptotically volume preserving: let $\mathcal{V}_{j}, \mathcal{V}_{\infty}:[0, \infty) \rightarrow[0, \infty)$ denote the respective volume functions $\mathcal{V}_{j}(t)=\operatorname{Vol}\left(\mathcal{M}_{t}^{j}\right)$ and $\mathcal{V}_{\infty}(t)=\operatorname{Vol}\left(\mathcal{M}_{t}^{\infty}\right)$; then the function $\mathcal{V}_{\infty}:[0, \infty) \rightarrow[0, \infty)$ is continuous and $\lim _{j \rightarrow \infty} \mathcal{V}_{j}=\mathcal{V}_{\infty}$, with uniform convergence on compact subsets of $[0, \infty)$.

Furthermore,

(a) the scalar curvature function $R_{\infty}: \mathcal{M}_{\leqslant T}^{\infty} \rightarrow \mathbb{R}$ is bounded below and proper for all $T \geqslant 0$;

(b) $\mathcal{M}^{\infty}$ satisfies the Hamilton-Ivey pinching condition of (A.14);

(c) $\mathcal{M}^{\infty}$ is $\varkappa$-non-collapsed below scale $\varepsilon$, and satisfies the $r$-canonical neighborhood assumption, where $\varkappa$ and $r$ are the aforementioned parameters from Ricci flow with surgery.

Theorem 1.3 may be compared with other convergence results such as Hamilton's compactness theorem [34] and its variants [44, Appendix E], as well as analogous results for sequences of Riemannian manifolds. All of these results require uniform bounds on curvature in regions of a given size around a basepoint, which we do not have. Instead, our approach is based on the fact that in a 3-dimensional Ricci flow with surgery, the scalar curvature controls the local geometry. We first prove a general pointed compactness result for sequences of (possibly incomplete) Riemannian manifolds whose local geometry is governed by a control function. We then apply this general compactness result in the case when the Riemannian manifolds are the spacetimes of Ricci flows with surgery, and the control functions are constructed from the scalar curvature functions. To obtain conditions (1)-(3) of Theorem 1.3, we have to rule out the possibility that part of the spacetime with controlled time and scalar curvature escapes to infinity, i.e. is not seen in the pointed limit. This is done by means of a new estimate on the spacetime geometry of a Ricci flow with surgery; see Proposition 3.3 below.

Motivated by the conclusion of Theorem 1.3, we make the following definition.

Definition 1.4. A Ricci flow spacetime $\left(\mathcal{M}, \mathfrak{t}, \partial_{\mathfrak{t}}, g\right)$ is a singular Ricci flow if it is 4-dimensional, the initial time slice $\mathcal{M}_{0}$ is a compact normalized Riemannian manifold 
and

(a) the scalar curvature function $R: \mathcal{M}_{\leqslant T} \rightarrow \mathbb{R}$ is bounded below and proper for all $T \geqslant 0$

(b) $\mathcal{M}$ satisfies the Hamilton-Ivey pinching condition of (A.14);

(c) for a global parameter $\varepsilon>0$ and decreasing functions $\varkappa, r:[0, \infty) \rightarrow(0, \infty)$, the spacetime $\mathcal{M}$ is $\varkappa$-non-collapsed below scale $\varepsilon$ in the sense of $\S$ A.4, and satisfies the $r$-canonical neighborhood assumption in the sense of $\S \mathrm{A} .8$.

Although conditions (b) and (c) in Definition 1.4 are pointwise conditions imposed everywhere, we will show elsewhere that $\mathcal{M}$ is a singular Ricci flow if (b) and (c) are only assumed to hold outside of some compact subset of $\mathcal{M}_{\leqslant T}$, for all $T \geqslant 0$. Thus (b) and (c) can be viewed as asymptotic conditions at infinity for a Ricci flow defined on a non-compact spacetime. Condition (a) implies that, if a spatial slice is non-compact, then the scalar curvature tends to infinity as one approaches an end; this latter property compensates for the possible lack of completeness.

With this definition, Perelman's existence theorem for Ricci flow with surgery and Theorem 1.3 immediately imply the following result.

COROllary 1.5. If $\left(M, g_{0}\right)$ is a compact normalized Riemannian 3-manifold, then there exists a singular Ricci flow having initial condition $\left(M, g_{0}\right)$ with parameter functions and $r$ as in Theorem 1.3.

From the PDE viewpoint, flow with surgery is a regularization of Ricci flow, while singular Ricci flows may be considered to be generalized solutions to Ricci flow. In this language, Corollary 1.5 gives the existence of generalized solutions by means of a regularization procedure. One can compare this with the existence proof for Brakke flows in [9], [43] or level set flows in [15], [25].

The existence assertion in Corollary 1.5 leads to the following uniqueness question.

Question 1.6. If two singular Ricci flows have isometric initial conditions, are the underlying Ricci flow spacetimes the same, up to diffeomorphism?

An affirmative answer would confirm Perelman's expectation that Ricci flow with surgery should converge to a canonical flow through singularities, as it would imply that, if one takes a fixed initial condition in Theorem 1.3, then one would have convergence without having to pass to a subsequence. Having such a uniqueness result, in conjunction with Theorem 1.3, would closely parallel the results of [10], [37], [39], [47] that 2-convex mean-curvature flow with surgery converges to level set flow when the surgery parameters tend to zero. 


\section{The structure of singular Ricci flows}

The asymptotic conditions in the definition of a singular Ricci flow have a number of implications which we analyze in this paper. In addition to clarifying the structure of limits of Ricci flows with surgery as in Theorem 1.3, the results indicate that singular Ricci flows are well-behaved objects from geometric and analytical points of view.

To analyze the geometry of Ricci flow spacetimes, we use two different Riemannian metrics.

Definition 1.7. Let $\left(\mathcal{M}, \mathfrak{t}, \partial_{\mathfrak{t}}, g\right)$ be a Ricci flow spacetime. The spacetime metric on $\mathcal{M}$ is the Riemannian metric $g_{\mathcal{M}}=\hat{g}+d \mathfrak{t}^{2}$, where $\hat{g}$ is the extension of $g$ to a quadratic form on $T \mathcal{M}$ such that $\partial_{\mathrm{t}} \in \operatorname{ker}(\hat{g})$. The quasi-parabolic metric on $\mathcal{M}$ is the Riemannian metric

$$
g_{\mathcal{M}}^{\mathrm{qp}}=\left(1+R^{2}\right)^{1 / 2} \hat{g}+\left(1+R^{2}\right) d \mathfrak{t}^{2} .
$$

For the remainder of the introduction, unless otherwise specified, $\left(\mathcal{M}, \mathfrak{t}, \partial_{\mathfrak{t}}, g\right)$ will denote a fixed singular Ricci flow.

Conditions (b) and (c) of Definition 1.4 imply that the scalar curvature controls the local geometry of the singular Ricci flow. This has several implications.

- (High-curvature regions in singular Ricci flows are topologically standard) For every $t$, the superlevel set $\left\{x \in \mathcal{M}_{t}: R(x) \geqslant r^{-2}(t)\right\}$ is contained in a disjoint union of connected components whose diffeomorphism types come from a small list of possibilities, with well-controlled local geometry. In particular, each connected component $C$ of $\mathcal{M}_{t}$ has finitely many ends, and passing to the metric completion $\bar{C}$ adds at most one point for each end (Proposition 5.16).

- (Bounded geometry) The spacetime metric $g_{\mathcal{M}}$ has bounded geometry at the scale defined by the scalar curvature, while the quasi-parabolic metric $g_{\mathcal{M}}^{\mathrm{qp}}$ is complete and has bounded geometry in the usual sense - the injectivity radius is bounded below, and all derivatives of curvature are uniformly bounded (Lemma 5.13).

The local control on geometry also leads to a compactness property for singular Ricci flows:

- (Compactness) If one has a sequence $\left\{\left(\mathcal{M}^{j}, \mathfrak{t}_{j}, \partial_{\mathfrak{t}_{j}}, g_{j}\right)\right\}_{j=1}^{\infty}$ of singular Ricci flows with a fixed choice of functions in Definition 1.4, and the initial metrics $\left\{\left(\mathcal{M}_{0}^{j}, g_{j}(0)\right)\right\}_{j=1}^{\infty}$ form a precompact set in the smooth topology, then a subsequence converges in the sense of Theorem 1.3 (Proposition 5.22).

The proof of the compactness result is similar to the proof of Theorem 1.3. We also have global results concerning the scalar curvature and volume:

- (Scalar curvature and volume control) For any $T<\infty$, the scalar curvature is integrable on $\mathcal{M}_{\leqslant T}$. The volume function $\mathcal{V}(t)=\operatorname{vol}\left(\mathcal{M}_{t}\right)$ is absolutely continuous and has 
a locally bounded upper-right derivative. The usual formula holds for volume evolution:

$$
\mathcal{V}\left(t_{1}\right)-\mathcal{V}\left(t_{0}\right)=-\int_{\mathcal{M}_{\left[t_{0}, t_{1}\right]}} R d \operatorname{vol}_{g_{\mathcal{M}}}
$$

for all $0 \leqslant t_{0} \leqslant t_{1}<\infty$ (Corollary 7.7).

Elsewhere we will discuss the structure of the completion of the spacetime, and will also show:

- (Refined scalar curvature and volume estimates) For all $p \in(0,1)$ and all $t$, the scalar curvature is $L^{p}$ on $\mathcal{M}_{t}$. The volume $\mathcal{V}(t)$ is locally $\alpha$-Hölder in $t$ for some exponent $\alpha \in(0,1)$.

To describe the next results, we introduce the following definitions.

Definition 1.9. A path $\gamma: I \rightarrow \mathcal{M}$ is time-preserving if $\mathfrak{t}(\gamma(t))=t$ for all $t \in I$. The worldline of a point $m \in \mathcal{M}$ is the maximal time-preserving integral curve $\gamma: I \rightarrow \mathcal{M}$ of the time vector field $\partial_{\mathfrak{t}}$, which passes through $m$.

If $\gamma: I \rightarrow \mathcal{M}$ is a worldline, then we may have $\sup I<\infty$. In this case, the scalar curvature blows up along $\gamma(t)$ as $t \rightarrow \sup I$, and the worldline encounters a singularity. An example would be a shrinking round space form, or a neck pinch. A worldline may also encounter a singularity going backward in time.

Definition 1.10. A worldline $\gamma: I \rightarrow \mathcal{M}$ is bad if inf $I>0$, i.e. if it is not defined at $t=0$.

Among our structural results, perhaps the most striking is the following.

Theorem 1.11. Suppose that $\left(\mathcal{M}, \mathfrak{t}, \partial_{\mathfrak{t}}, g\right)$ is a singular Ricci flow and $t \geqslant 0$. If $C$ is a connected component of $\mathcal{M}_{t}$, then only finitely many points in $C$ have bad worldlines. Moreover, if $\gamma: I \rightarrow \mathcal{M}$ is a bad worldline, then for $t \in I$ sufficiently close to inf $I, \gamma(t)$ lies in a cap region of $\mathcal{M}_{t}$.

As an illustration of the theorem, consider a singular Ricci flow that undergoes a generic neck pinch at time $t_{0}$, so that the time slice $\mathcal{M}_{t_{0}}$ has two ends ( $\varepsilon$-horns in Perelman's language) which are instantly capped off when $t>t_{0}$. In this case, the theorem asserts that only finitely many (in this case two) worldlines emerge from the singularity. See Figure 1, where the bad wordlines are indicated by dashed curves. (The point in the figure where the two dashed curves meet is not in the spacetime.)

A key ingredient in the proof of Theorem 1.11 is a new stability property of neck regions in $\varkappa$-solutions. We recall that $\varkappa$-solutions are the class of ancient Ricci flows used to model the high-curvature part of Ricci flows with surgery. We state the stability property loosely as follows, and refer the reader to $\S 6$ for more details. 


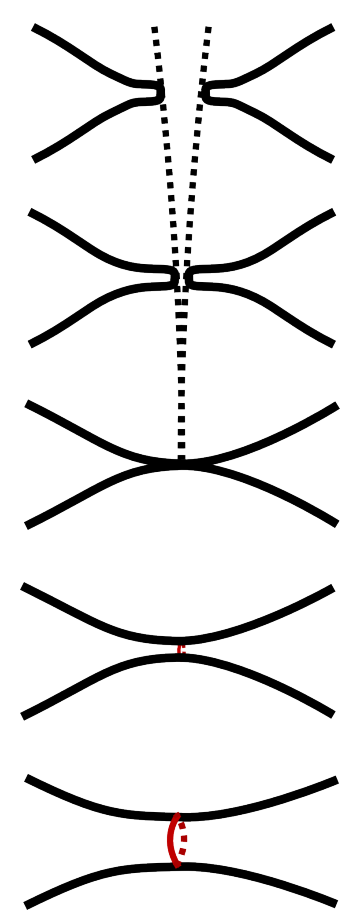

Figure 1.

TheOREM 1.12. (Neck stability) Let $\mathcal{M}$ be a non-compact $\varkappa$-solution other than the shrinking round cylinder. If $\gamma: I \rightarrow \mathcal{M}$ is a worldline and $\mathcal{M}_{t_{1}}$ is sufficiently neck-like at $\gamma\left(t_{1}\right)$, then as $t \rightarrow-\infty$, the time slice $\mathcal{M}_{t}$ looks more and more neck-like at $\gamma(t)$. Here the notion of neck-likeness is scale invariant.

An easy but illustrative case is the Bryant soliton, in which worldlines other than the tip itself move away from the tip (in the scale-invariant sense) as one goes backward in time.

As mentioned above, Theorem 1.11 is used in the proof of (1.8) and the properties of volume.

We mention some connectedness properties of singular Ricci flows.

- (Paths back to $\mathcal{M}_{0}$ avoiding high-curvature regions) Any point $m \in \mathcal{M}$ can be joined to the initial time slice $\mathcal{M}_{0}$ by a time-preserving curve $\gamma:[0, \mathfrak{t}(m)] \rightarrow \mathcal{M}$, along which $\max \{R(\gamma(t)): t \in[0, \mathfrak{t}(m)]\}$ is bounded in terms of $R(m)$ and $\mathfrak{t}(m)$ (Proposition 5.21).

- (Backward stability of components) If $\gamma_{0}, \gamma_{1}:\left[t_{0}, t_{1}\right] \rightarrow \mathcal{M}$ are time-preserving curves such that $\gamma_{0}\left(t_{1}\right)$ and $\gamma_{1}\left(t_{1}\right)$ lie in the same connected component of $\mathcal{M}_{t_{1}}$, then $\gamma_{0}(t)$ and $\gamma_{1}(t)$ lie in the same component of $\mathcal{M}_{t}$ for all $t \in\left[t_{0}, t_{1}\right]$ (Proposition 5.17). 


\section{Related work}

We now mention some other work that falls into the broad setting of Ricci flow with singular structure.

A number of authors have considered Ricci flow with low-regularity initial conditions, studying existence and/or uniqueness, and instantaneous improvement of regularity [12], [32], [33], [46], [62], [67]-[69], [75]-[76]. Ricci flow with persistent singularities has been considered in the case of orbifold Ricci flow, and Ricci flow with conical singularities [13], [14], [17], [19], [22], [36], [45], [50]-[52], [59], [77], [80], [83], [84]

Passing to flows through singularities, Feldman-Ilmanen-Knopf noted that in the non-compact Kähler setting, there are some natural examples of flows through singularities which consist of a shrinking gradient soliton that has a conical limit at time zero, which transmutes into an expanding gradient soliton [30]. Closer in spirit to this paper, Angenent-Caputo-Knopf constructed a rotationally invariant Ricci flow through singularities starting with a metric on $S^{n+1}$ [3]. They showed that the rotationally invariant neck pinches from [4], which have a singular limit as $t$ approaches zero from below, may be continued as a smooth Ricci flow on two copies of $S^{n+1}$ for $t>0$. The paper [3] also showed that the forward evolution has a unique asymptotic profile near the singular point in spacetime.

There has been much progress on flowing through singularities in the Kähler setting. For a flow on a projective variety with log terminal singularities, Song-Tian [71] showed that the flow can be continued through the divisorial contractions and flips of the minimal model program. We refer to [71] for the precise statements. The paper [27] has related results, but uses a viscosity solution approach instead of the regularization scheme in [71]. The fact that the Kähler-Ricci flow-like the Kähler-Einstein equation — can be reduced to a scalar equation, to which comparison principles may be applied, is an important simplifying feature of Kähler-Ricci flow that is not available in the non-Kähler case.

Recently, Haslhofer-Naber gave alternative characterizations of the Ricci flow using stochastic analysis [38]; they also announced an extension to evolving metric measure spaces.

\section{Concluding remarks}

The work in this paper is related to the question of whether there is a good notion of a generalized solution to the Ricci flow equation. The results here show that singular Ricci flows give an answer in the 3-dimensional case, and in the 4-dimensional case under the assumption of non-negative isotropic curvature.

By using different quantities to control the geometry, it may be possible to work 
with flows satisfying weaker curvature conditions. The general higher-dimensional case, however, is mysterious, and finding a good notion of generalized solution is an intriguing question. For mean-curvature flows with arbitrary smooth initial conditions, the situation is somewhat similar.

As mentioned before, some of the technical methods in this paper, such as a compactness result for possibly incomplete Riemannian manifolds, and a dynamical analysis of ancient solutions, may be useful for other problems in geometric analysis.

\section{Organization of the paper}

The remainder of the paper is broken into two parts. Part I, which is composed of $\S \S 2-4$, is primarily concerned with the proof of the convergence result (Theorem 1.3). Part II, which is composed of $\S \S 5-7$, deals with results on singular Ricci flows. In addition, there are three appendices.

We now describe the contents section by section.

$\S 2$ gives a general pointed compactness result for Riemannian manifolds and spacetimes, whose geometry are locally controlled as a function of some auxiliary function. $\S 3$ develops some properties of Ricci flow with surgery; it is aimed at showing that the Ricci flow spacetime associated with a Ricci flow with surgery has locally controlled geometry in the sense of $\S 2$. $\S 4$ applies the two preceding sections to give the proof of Theorem 1.3.

$\S 5$ establishes some foundational results about singular Ricci flows, concerning scalar curvature and volume, as well as some results involving the structure of the high-curvature region. $\S 6$ proves that neck regions in $\varkappa$-solutions have a stability property when going backward in time. $\$ 7$ proves Theorem 1.11, concerning the finiteness of the number of bad worldlines, and gives several applications.

Appendix A collects a variety of background material about Ricci flows and Ricci flows with surgery; the reader may wish to quickly peruse this, before proceeding to the body of the paper. Appendix B extends Proposition 6.10 to general $\varkappa$-solutions. Appendix $\mathrm{C}$ extends the results of the paper to 4-dimensional Ricci flow with non-negative isotropic curvature.

\section{Notation and terminology}

We refer the reader to $\S \mathrm{A} .1$ for notation and terminology. All manifolds that arise will be taken to be orientable.

We thank the referee for a careful reading and helpful comments. 


\section{Part I}

\section{Compactness for spaces of locally controlled geometries}

In this section we prove a compactness result (Theorem 2.4) for sequences of controlled Riemannian manifolds. In $\S 2.4$ we extend the theorem to a compactness result for spacetimes, meaning Riemannian manifolds equipped with time functions and time vector fields.

\subsection{Compactness of the space of locally controlled Riemannian manifolds}

We will need a sequential compactness result for sequences of Riemannian manifolds which may be incomplete, but whose local geometry (injectivity radius and all derivatives of curvature) is bounded by a function of an auxiliary function $\psi: M \rightarrow[0, \infty)$. A standard case of this is the sequential compactness for sequences $\left\{\left(M_{j}, g_{j}, \star_{j}\right)\right\}$ of Riemannian $r$ balls, assuming that the distance function $d_{j}\left(\star_{j}, \cdot\right): M_{j} \rightarrow[0, r)$ is proper, and that the geometry is bounded in terms of $d_{j}\left(\star_{j}, \cdot\right)$.

Fix a smooth decreasing function $\mathfrak{r}:[0, \infty) \rightarrow(0,1]$ and smooth increasing functions $C_{k}:[0, \infty) \rightarrow[1, \infty)$ for all $k \geqslant 0$.

Definition 2.1. Suppose that $(M, g)$ is a Riemannian manifold equipped with a function $\psi: M \rightarrow[0, \infty)$.

Given $A \in[0, \infty]$, a tensor field $\xi$ is $(\psi, A)$-controlled if, for all $m \in \psi^{-1}([0, A))$ and $k \geqslant 0$, we have

$$
\left\|\nabla^{k} \xi(m)\right\| \leqslant C_{k}(\psi(m))
$$

If in addition $\psi$ is smooth, then we say that the tuple $(M, g, \psi)$ is $(\psi, A)$-controlled if

(1) the injectivity radius of $M$ at $m$ is at least $\mathfrak{r}(\psi(m))$ for all $m \in \psi^{-1}([0, A))$,

(2) the tensor field $\mathrm{Rm}$, and $\psi$ itself, are $(\psi, A)$-controlled.

Note that, if $A=\infty$, then $\psi^{-1}([0, A))$ is all of $M$, so there are quantitative bounds on the geometry at each point of $M$. Note also that the value of a control function may not reflect the actual bounds on the geometry, in the sense that the geometry may be more regular near $m$ than the value of $\psi(m)$ suggests. This creates flexibility in choosing a control function, which is useful in applications below.

Example 2.2. Suppose that $(M, g, \star)$ is a complete pointed Riemannian manifold. Put $\psi(m)=d(\star, m)$. Then $\mathrm{Rm}$ is $(\psi, A)$-controlled if and only if for all $r \in(0, A)$ and $k \geqslant 0$ we have $\left\|\nabla^{k} \mathrm{Rm}\right\| \leqslant C_{k}(r)$ on $B(\star, r)$. 
Example 2.3. Suppose that $\psi$ has constant value $c>0$. If $A \leqslant c$, then $(M, g, \psi)$ is vacuously $(\psi, A)$-controlled. If $A>c$, then $(M, g, \psi)$ is $(\psi, A)$-controlled if and only if for all $m \in M$ we have $\operatorname{inj}(m) \geqslant \mathfrak{r}(c)$ and $\left\|\nabla^{k} \operatorname{Rm}(m)\right\| \leqslant C_{k}(c)$.

There are compactness results in Riemannian geometry saying that one can extract a subsequential limit from a sequence of complete pointed Riemannian manifolds having uniform local geometry. This last condition means that, for each $r>0$, one has quantitative uniform bounds on the geometry of the $r$-ball around the basepoint; cf. [34, Theorem 2.3]. In such a case, one can think of the distance from the basepoint as a control function. We will give a compactness theorem for Riemannian manifolds (possibly incomplete) equipped with more general control functions.

For notation, if $\Phi: U \rightarrow V$ is a diffeomorphism, then we will write $\Phi_{*}$ for both the push-forward action of $\Phi$ on contravariant tensor fields on $U$, and the pull-back action of $\Phi^{-1}$ on covariant tensor fields on $U$.

THEOREM 2.4. (Compactness for controlled manifolds) Let

$$
\left\{\left(M_{j}, g_{j}, \star_{j}, \psi_{j}\right)\right\}_{j=1}^{\infty}
$$

be a sequence of pointed tuples which are $\left(\psi_{j}, A_{j}\right)$-controlled, where $\lim _{j \rightarrow \infty} A_{j}=\infty$ and $\sup _{j} \psi_{j}\left(\star_{j}\right)<\infty$. Then, after passing to a subsequence, there are a pointed $\left(\psi_{\infty}, \infty\right)$ controlled tuple

$$
\left(M_{\infty}, g_{\infty}, \star_{\infty}, \psi_{\infty}\right)
$$

and a sequence of diffeomorphisms

$$
\left\{M_{j} \supset U_{j} \stackrel{\Phi^{j}}{\longrightarrow} V_{j} \subset M_{\infty}\right\}_{j=1}^{\infty}
$$

such that the following conditions hold:

(1) given $A, r<\infty$, for all sufficiently large $j$ the open set $U_{j}$ contains the ball $B\left(\star_{j}, r\right)$ in the Riemannian manifold $\left(\psi_{j}^{-1}([0, A)), g_{j}\right)$, and likewise $V_{j}$ contains the ball $B\left(\star_{\infty}, r\right)$ in the Riemannian manifold $\left(\psi_{\infty}^{-1}([0, A)), g_{\infty}\right)$;

(2) given $\varepsilon>0$ and $k \geqslant 0$, for all sufficiently large $j$ we have

$$
\left\|\Phi_{*}^{j} g_{j}-g_{\infty}\right\|_{C^{k}\left(V_{j}\right)}<\varepsilon
$$

and

$$
\left\|\Phi_{*}^{j} \psi_{j}-\psi_{\infty}\right\|_{C^{k}\left(V_{j}\right)}<\varepsilon
$$

(3) $M_{\infty}$ is connected and, in particular, every $x \in M_{\infty}$ belongs to $V_{j}$ for $j$ large.

We will give the proof of Theorem 2.4 in $\S 2.3$. We first describe some general results about controlled Riemannian manifolds. 


\subsection{Some properties of controlled Riemannian manifolds}

One approach to proving Theorem 2.4 would be to imitate what one does when one has curvature and injectivity radius bounds on $r$-balls, replacing the control function based on distance to the basepoint by the control function $\psi$. While this could be done, it would be somewhat involved. Instead, we will perform a conformal change on the Riemannian manifolds in order to put ourselves in a situation where the geometry is indeed controlled by the distance from the basepoint. We then take a subsequential limit of the conformally changed metrics, and at the end perform another conformal change to get a subsequential limit of the original sequence.

Let $(M, g, \psi)$ be $(\psi, A)$-controlled. Put

$$
\tilde{g}=\left(\frac{C_{1}}{\mathfrak{r}} \circ \psi\right)^{2} g .
$$

We will only consider $\tilde{g}$ on the subset $\psi^{-1}([0, A))$, where it is smooth. The next two lemmas are about $g$-balls and $\tilde{g}$-balls.

LEMma 2.6. For each finite $a \in(0, A]$, each $\star \in \psi^{-1}([0, a))$ and each $r<\infty$, there is some $R=R(a, r)<\infty$ so that the ball $B_{g}(\star, r)$ in the Riemannian manifold $\left(\psi^{-1}([0, a)), g\right)$ is contained in the ball $B_{\tilde{g}}(\star, R)$ in the Riemannian manifold $\left(\psi^{-1}([0, a)), \tilde{g}\right)$.

Proof. On $\psi^{-1}([0, a))$ we have

$$
\tilde{g} \leqslant\left(\frac{C_{1}(a)}{\mathfrak{r}(a)}\right)^{2} g
$$

Thus, any path in $\psi^{-1}([0, a))$ with $g$-length at most $r$ has $\tilde{g}$-length at most $C_{1}(a) r / \mathfrak{r}(a)$, so we may take $R=C_{1}(a) r / \mathfrak{r}(a)$.

Let $\star$ be a basepoint in $\psi^{-1}([0, A))$.

Lemma 2.7. For all $R \in(0, A-\psi(\star))$, the ball $B_{\tilde{g}}(\star, R)$ in the Riemannian manifold $\left(\psi^{-1}([0, A)), \tilde{g}\right)$ is contained in $\psi^{-1}([0, \psi(\star)+R))$.

Proof. Given $m \in B_{\tilde{g}}(\star, R)$, let $\gamma:[0, L] \rightarrow \psi^{-1}([0, A))$ be a smooth path from $\star$ to $m$ with unit $\tilde{g}$-speed and $\tilde{g}$-length $L \in(0, R)$. Then,

$$
\begin{aligned}
\psi(m)-\psi(\star) & =\int_{0}^{L} \frac{d}{d t} \psi(\gamma(t)) d t \leqslant \int_{0}^{L}|d \psi| \tilde{g}(\gamma(t)) d t \\
& =\int_{0}^{L} \frac{\mathfrak{r}}{C_{1}}(\psi(\gamma(t)))|d \psi|_{g}(\gamma(t)) d t \leqslant \int_{0}^{L} \mathfrak{r}(\psi(\gamma(t))) d t \leqslant \int_{0}^{L} 1 d t=L .
\end{aligned}
$$

The lemma follows. 
We now look at completeness properties of $\tilde{g}$-balls.

Lemma 2.8. For all $R \in(0, A-\psi(\star))$, the ball $B_{\tilde{g}}(\star, R)$ in the Riemannian manifold $\left(\psi^{-1}([0, A)), \tilde{g}\right)$ has compact closure in $\psi^{-1}([0, A))$.

Proof. Choose $R^{\prime} \in(R, A-\psi(\star))$. From [5, Chapter 1, Theorem 2.4], it suffices to show that any $\tilde{g}$-unit speed geodesic $\gamma:[0, L) \rightarrow \psi^{-1}([0, A))$ with $\gamma(0)=\star$, having $\tilde{g}$ length $L \in\left(0, R^{\prime}\right)$, can be extended to $[0, L]$. From Lemma $2.7, \gamma([0, L)) \subset \psi^{-1}([0, \psi(\star)+$ $\left.R^{\prime}\right)$ ). Hence, the $g$-injectivity radius along $\gamma([0, L))$ is bounded below by $\left.\mathfrak{r}\left(\psi(\star)+R^{\prime}\right)\right)$. For large $K$, the points $\{\gamma(L-1 / k)\}_{k=K}^{\infty}$ form a Cauchy sequence in $\left(\psi^{-1}([0, A)), d_{\tilde{g}}\right)$. As $d_{\tilde{g}}$ and $d_{g}$ are bi-Lipschitz on $\psi^{-1}\left(\left[0, \psi(\star)+R^{\prime}\right)\right)$, the sequence is also Cauchy in $\left(\psi^{-1}([0, A)), d_{g}\right)$. From the uniform positive lower bound on the $g$-injectivity radius at $\gamma(L-1 / k)$, there is a limit in $\psi^{-1}([0, A))$. The lemma follows.

Corollary 2.9. If $A=\infty$, then $(M, \tilde{g})$ is complete.

Proof. This follows from Lemma 2.8 and [5, Chapter 1, Theorem 2.4].

Finally, we give bounds on the geometry of $\tilde{g}$-balls.

LEMMA 2.10. Given $\mathfrak{r},\left\{C_{k}\right\}_{k=1}^{\infty}$ and $S<\infty$, there exist a smooth decreasing function $\tilde{\mathfrak{r}}:[0, \infty) \rightarrow(0,1]$ and smooth increasing functions $\widetilde{C}_{k}:[0, \infty) \rightarrow[1, \infty), k \geqslant 0$, with the following properties. Suppose that $(M, g, \psi)$ is $(\psi, A)$-controlled and $\psi(\star) \leqslant S$. Then,

(a) the tensor field $\operatorname{Rm}_{\tilde{g}}$ and the function $\psi$ are $\left(d_{\tilde{g}}(\star, \cdot), A-S\right)$-controlled on the Riemannian manifold $\left(\psi^{-1}([0, A)), \tilde{g}\right)$ (in terms of the functions $\left\{\widetilde{C}_{k}\right\}_{k=1}^{\infty}$ );

(b) if $R<A-S-1$, then $\operatorname{inj}_{\tilde{g}} \geqslant \tilde{\mathfrak{r}}(R)$ pointwise on $B_{\tilde{g}}(\star, R)$.

Proof. Conclusion (a) (along with the concomitant functions $\left\{\widetilde{C}_{k}\right\}_{k=1}^{\infty}$ ) follows from Lemma 2.7, the assumption that $\operatorname{Rm}_{g}$ and $\psi$ are $(\psi, A)$-controlled, and the formula for the Riemannian curvature of a conformally changed metric.

To prove (b), suppose that $R<A-S-1$ and $m \in B_{\tilde{g}}(\star, R)$. Since $d_{\tilde{g}}$ and $d_{g}$ are biLipschitz on the ball $B_{\tilde{g}}(\star, R+1)$ in the Riemannian manifold $\left(\psi^{-1}([0, R+1+S)), \tilde{g}\right)$, we can find $\varepsilon=\varepsilon\left(R, S,\left\{C_{k}\right\}\right)>0$ such that $B_{g}(m, \varepsilon) \subset B_{\tilde{g}}(m, 1) \subset B_{\tilde{g}}(\star, R+1)$. Since we have a $g$-curvature bound on $B_{\tilde{g}}(\star, R+1)$ and a lower $g$-injectivity radius bound at $m$, we obtain a lower volume bound $\operatorname{vol}\left(B_{g}(m, \varepsilon), g\right) \geqslant v_{0}=v_{0}\left(R, S, \mathfrak{r},\left\{C_{k}\right\}\right)>0$. Since $g$ and $\tilde{g}$ are relatively bounded on $B_{g}(m, \varepsilon)$, this gives a lower volume bound $\operatorname{vol}\left(B_{\tilde{g}}(m, 1), \tilde{g}\right) \geqslant$ $v_{1}=v_{1}\left(R, S, \mathfrak{r},\left\{C_{k}\right\}\right)>0$. Using the curvature bound of part (a) and [11, Theorem 4.7], we obtain a lower bound $\operatorname{inj}_{\tilde{g}}(m) \geqslant i_{0}=i_{0}\left(R, S, \mathfrak{r},\left\{C_{k}\right\}\right)>0$. This proves the lemma.

\subsection{Proof of Theorem 2.4}

Put $\tilde{g}_{j}=\left(C_{1} / \mathfrak{r} \circ \psi_{j}\right)^{2} g_{j}$. Consider the tuple $\left(M_{j}, \tilde{g}_{j}, \star_{j}, \psi_{j}\right)$. Recall that $\lim _{j \rightarrow \infty} A_{j}=\infty$. 
For the moment, we replace the index $j$ by the index $l$. Using Lemmas 2.8 and 2.10, and a standard compactness theorem ([34, Theorem 2.3]), after passing to a subsequence we can find a complete pointed Riemannian manifold $\left(M_{\infty}, g_{\infty}, \star_{\infty}\right)$, domains $\widetilde{U}_{l} \subset M_{l}$ and $\widetilde{V}_{l} \subset M_{\infty}$, and diffeomorphisms $\tilde{\Phi}^{l}: \widetilde{U}_{l} \rightarrow \widetilde{V}_{l}$ such that

(a) $\star_{l} \in \widetilde{U}_{l}$;

(b) $\star_{\infty} \in \widetilde{V}_{l}$;

(c) $\widetilde{V}_{l}$ has compact closure;

(d) for any compact set $K \subset M_{\infty}$, we have $K \subset \widetilde{V}_{l}$ for all sufficiently large $l$;

(e) given $\varepsilon>0$ and $k \geqslant 0$, we have

$$
\left\|\tilde{\Phi}_{*}^{l} \tilde{g}_{l}-\tilde{g}_{\infty}\right\|_{C^{k}\left(\tilde{V}_{l}, \tilde{g}_{\infty}\right)}<\varepsilon
$$

for all sufficiently large $l$.

Using Lemma 2.10 again, after passing to a further subsequence if necessary, we can assume that there is a smooth $\psi_{\infty}$ on $M_{\infty}$ such that for all $\varepsilon>0$ and $k \geqslant 0$ we have

$$
\left\|\tilde{\Phi}_{*}^{l} \psi_{l}-\psi_{\infty}\right\|_{C^{k}\left(\widetilde{V}_{l}, \tilde{g}_{\infty}\right)}<\varepsilon
$$

for all sufficiently large $l$. Put

$$
g_{\infty}=\left(\frac{C_{1}}{\mathfrak{r}} \circ \psi_{\infty}\right)^{-2} \tilde{g}_{\infty} .
$$

We claim that, if the sequence $\left\{l_{j}\right\}_{j=1}^{\infty}$ increases rapidly enough, then the conclusions of the theorem can be made to hold with $V_{j}=B_{\tilde{g}_{\infty}}\left(\star_{\infty}, j\right) \subset M^{\infty}, U_{j}=\left(\tilde{\Phi}^{l_{j}}\right)^{-1}\left(V_{j}\right) \subset M^{l_{j}}$ and $\Phi^{j}=\left.\tilde{\Phi}^{l_{j}}\right|_{U_{j}}$. To see this, we note the following facts.

- Given $A, r<\infty$, Lemma 2.6 implies that the ball $B\left(\star_{\infty}, r\right)$ in the Riemannian manifold $\left(\psi_{\infty}^{-1}([0, A)), g_{\infty}\right)$ will be contained in $V_{j}$ for all sufficiently large $j$.

- If the sequence $\left\{l_{j}\right\}_{j=1}^{\infty}$ increases rapidly enough, then for large $j$ the map $\Phi^{j}$ is arbitrarily close to an isometry, and $\left(\Phi^{j}\right)_{*} \psi_{j}$ is arbitrarily close to $\psi_{\infty}$ on $V_{j}$. Hence, given $A, r<\infty$, the ball $B\left(\star_{j}, r\right)$ in the Riemannian manifold $\left(\psi_{j}^{-1}([0, A)), g_{j}\right)$ will be contained in $U_{j}$ for all sufficiently large $j$, so conclusion (1) of the theorem holds.

- The metrics $g_{\infty}$ and $\tilde{g}_{\infty}$ are bi-Lipschitz on $V_{j}$. Then, if the sequence $\left\{l_{j}\right\}_{j=1}^{\infty}$ increases rapidly enough, conclusion (2) of the theorem can be made to hold.

- Conclusion (3) of the theorem follows from the definition of $V_{j}$.

This proves Theorem 2.4.

\subsection{Compactness of the space of locally controlled spacetimes}

We now apply Theorem 2.4 to prove a compactness result for spacetimes. 
Definition 2.12. A spacetime is a Riemannian manifold $\left(\mathcal{M}, g_{\mathcal{M}}\right)$ equipped with a submersion $\mathfrak{t}: \mathcal{M} \rightarrow \mathbb{R}$ and a smooth vector field $\partial_{\mathfrak{t}}$ such that $d \mathfrak{t}\left(\partial_{\mathfrak{t}}\right) \equiv 1$.

Given $A \in[0, \infty]$ and $\psi \in C^{\infty}(\mathcal{M})$, we say that the tuple $\left(\mathcal{M}, g_{\mathcal{M}}, \mathfrak{t}, \partial_{\mathfrak{t}}, \psi\right)$ is $(\psi, A)$ controlled if $\left(\mathcal{M}, g_{\mathcal{M}}, \psi\right)$ is $(\psi, A)$-controlled in the sense of Definition 2.1 and, in addition, the tensor fields $\mathfrak{t}$ and $\partial_{\mathfrak{t}}$ are $(\psi, A)$-controlled.

Theorem 2.13. (Compactness for controlled spacetimes) Let

$$
\left\{\left(\mathcal{M}^{j}, g_{\mathcal{M}^{j}}, \mathfrak{t}_{j},\left(\partial_{\mathfrak{t}}\right)_{j}, \star_{j}, \psi_{j}\right)\right\}_{j=1}^{\infty}
$$

be a sequence of pointed tuples which are $\left(\psi_{j}, A_{j}\right)$-controlled, where $\lim _{j \rightarrow \infty} A_{j}=\infty$ and $\sup _{j} \psi_{j}\left(\star_{j}\right)<\infty$. Then, after passing to a subsequence, there exists a pointed $\left(\psi_{\infty}, \infty\right)$ controlled tuple

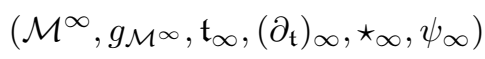

and a sequence of diffeomorphisms

$$
\left\{\mathcal{M}^{j} \supset U_{j} \stackrel{\Phi^{j}}{\longrightarrow} V_{j} \subset \mathcal{M}^{\infty}\right\}_{j=1}^{\infty}
$$

of open sets such that the following conditions are satisfied:

(1) given $A<\infty$ and $r<\infty$, for all sufficiently large $j$ the open set $U_{j}$ contains the ball $B\left(\star_{j}, r\right)$ in the Riemannian manifold $\left(\psi_{j}^{-1}([0, A)), g_{\mathcal{M}^{j}}\right)$, and likewise $V_{j}$ contains the ball $B\left(\star_{\infty}, r\right) \subset\left(\psi_{\infty}^{-1}([0, A)), g_{\mathcal{M}^{\infty}}\right)$.

(2) $\Phi^{j}$ exactly preserves the time functions, that is

$$
\mathfrak{t}_{\infty} \circ \Phi^{j}=\mathfrak{t}_{j} \quad \text { for all } j
$$

(3) $\Phi^{j}$ asymptotically preserves the tensor fields $g_{\mathcal{M}^{j}},\left(\partial_{\mathfrak{t}}\right)_{j}$, and $\psi_{j}$ : if

$$
\xi_{j} \in\left\{g_{\mathcal{M}^{j}},\left(\partial_{\mathfrak{t}}\right)_{j}, \psi_{j}\right\}
$$

and $\xi_{\infty}$ is the corresponding element of $\left\{g_{\mathcal{M}^{\infty}},\left(\partial_{\mathfrak{t}}\right)_{\infty}, \psi_{\infty}\right\}$, then for all $\varepsilon>0$ and $k \geqslant 0$ we have

$$
\left\|\Phi_{*}^{j} \xi_{j}-\xi_{\infty}\right\|_{C^{k}\left(V_{j}\right)}<\varepsilon
$$

for all sufficiently large $j$;

(4) $\mathcal{M}^{\infty}$ is connected and, in particular, every $x \in \mathcal{M}^{\infty}$ belongs to $V_{j}$ for large $j$.

Proof. Put

$$
\tilde{g}_{\mathcal{M}^{j}}=\left(\frac{C_{1}}{\mathfrak{r}} \circ \psi_{j}\right)^{2} g_{\mathcal{M}^{j}} .
$$

Consider the pointed spacetime $\left(\mathcal{M}^{j}, \tilde{g}_{\mathcal{M}^{j}}, \mathfrak{t}_{j},\left(\partial_{\mathfrak{t}}\right)_{j}, \star_{j}, \psi_{j}\right)$. 
Lemma 2.14. For every $A<\infty$, the tensor fields $\operatorname{Rm}_{\tilde{g}_{j}}, \mathfrak{t}_{j},\left(\partial_{t}\right)_{j}$, and $\psi_{j}$ are all $\left(d_{\tilde{g}_{\mathcal{M}^{j}}}\left(\star_{j}, \cdot\right), A\right)$-controlled for all large $j$, in the sense of Lemma 2.10 .

Proof. This follows as in the proof of Lemma 2.10.

We follow the proof of Theorem 2.4, up to the construction of $\tilde{\Phi}^{l}: \widetilde{U}_{l} \rightarrow \widetilde{V}_{l}$ and $\psi_{\infty}$. Using Lemma 2.14, after passing to a further subsequence if necessary, we may assume that there are smooth $\mathfrak{t}_{\infty}$ and $\left(\partial_{\mathfrak{t}}\right)_{\infty}$ on $\mathcal{M}^{\infty}$ such that, if $\xi_{l} \in\left\{\mathfrak{t}_{l},\left(\partial_{\mathfrak{t}}\right)_{l}, \psi_{l}\right\}$, then for all $\varepsilon>0$ and $k \geqslant 0$ we have

$$
\left\|\tilde{\Phi}_{*}^{l} \xi_{l}-\xi_{\infty}\right\|_{C^{k}\left(\widetilde{V}_{l}, \tilde{g}_{\mathcal{M} \infty}\right)}<\varepsilon
$$

for all sufficiently large $l$. Again, $\mathfrak{t}_{\infty}: \mathcal{M}^{\infty} \rightarrow \mathbb{R}$ is a submersion and $\left(\partial_{\mathfrak{t}}\right)_{\infty} \mathfrak{t}_{\infty}=1$. As before, put

$$
g_{\mathcal{M}^{\infty}}=\left(\frac{C_{1}}{\mathfrak{r}} \circ \psi_{\infty}\right)^{-2} \tilde{g}_{\mathcal{M}^{\infty}} .
$$

Let $\left\{\phi_{s}\right\}$ be the flow generated by $\left(\partial_{\mathfrak{t}}\right)_{\infty}$; this exists for at least a small time interval if the starting point is in a given compact subset of $M_{\infty}$. Put $V_{j}^{\prime}=B_{\tilde{g}_{\infty}}\left(\star_{\infty}, j\right)$. Then, there is some $\Delta_{j}>0$ so that $\left\{\phi_{s}\right\}$ exists on $V_{j}^{\prime}$ for $|s|<\Delta_{j}$. Given $l_{j} \gg 0$, put $U_{j}=\left(\tilde{\Phi}^{l_{j}}\right)^{-1}\left(V_{j}^{\prime}\right)$. Assuming that $l_{j}$ is large enough, we can define $\Phi^{j}: U_{j} \rightarrow \mathcal{M}^{\infty}$ by

$$
\Phi^{j}(m)=\phi_{\mathbf{t}_{j}(m)-\mathfrak{t}_{\infty}\left(\tilde{\Phi}^{\left.l_{j}(m)\right)}\right.}\left(\tilde{\Phi}^{l_{j}}(m)\right) .
$$

By construction, $\mathfrak{t}_{\infty}\left(\Phi^{j}(m)\right)=\mathfrak{t}_{j}(m)$, and so conclusion (2) of the theorem holds. If $l_{j}$ is large, then $\Phi^{j}$ will be a diffeomorphism to its image. Putting $V_{j}=\Phi^{j}\left(U_{j}\right)$, if $l_{j}$ is large enough, then $V_{j}$ can be made arbitrarily close to $V_{j}^{\prime}$. It follows that conclusions (1), (3) and (4) of the theorem hold.

Remark 2.15. In Hamilton's compactness theorem [34], the comparison map $\Phi^{j}$ preserves both the time function and the time vector field. In Theorem 2.13, the comparison map $\Phi^{j}$ preserves the time function, but the time vector field is only preserved asymptotically. This is good enough for our purposes.

\section{Properties of Ricci flows with surgery}

In this section, we prove several estimates for Ricci flows with surgery. These will be used in the proof of Theorem 1.3, to show that the sequence of Ricci flow spacetimes has the local control required for the application of the spacetime compactness theorem (Theorem 2.13).

The arguments in this section require familiarity with some basic properties of Ricci flow with surgery. For the reader's convenience, we have collected these properties in $\S$ A.8. The reader may wish to review this material, before proceeding. 
Let $\mathcal{M}$ be a Ricci flow with surgery in the sense of Perelman [44, §68]. As recalled in $\S$ A.9, the following parameters - or more precisely positive decreasing parameter functions - are associated with $\mathcal{M}$ :

- The canonical neighborhood scale function $r(t)>0$. We may assume that $r(0)<\frac{1}{10}$.

- The non-collapsing function $\varkappa(t)>0$.

- The parameter $\delta(t)>0$. This has a dual role: it is the quality of the surgery neck, and it enforces a scale buffer between the canonical neighborhood scale $r$, the intermediate scale $\varrho$ and the surgery scale $h$.

- The intermediate scale $\varrho(t)=\delta(t) r(t)$, which defines the threshold for discarding entire connected components at the singular time.

- The surgery scale $h(t)<\delta^{2}(t) r(t)$.

- The global parameter $\varepsilon>0$. This enters in the definition of a canonical neighborhood. For the Ricci flow with surgery to exist, a necessary condition is that $\varepsilon$ be small enough.

In this section, canonical neighborhoods are those defined for Ricci flows with surgery, as in [44, Definition 69.1]. The next lemma gives a sufficient condition for parabolic neighborhoods to be unscathed.

LEMma 3.1. Let $\mathcal{M}$ be a Ricci flow with surgery, with normalized initial condition. Given $T>\frac{1}{100}$, there are numbers $\mu=\mu(T) \in(0,1), \sigma=\sigma(T) \in(0,1), i_{0}=i_{0}(T)>0$ and $A_{k}=A_{k}(T)<\infty, k \geqslant 0$ which satisfy the following property: if $t \in\left(\frac{1}{100}, T\right]$ and $|R(x, t)|<$ $\mu \varrho(0)^{-2}-r(T)^{-2}$, put $Q=|R(x, t)|+r(t)^{-2}$, then

(1) the forward and backward parabolic balls

$$
P_{+}\left(x, t, \sigma Q^{-1 / 2}\right) \quad \text { and } \quad P_{-}\left(x, t, \sigma Q^{-1 / 2}\right)
$$

are unscathed;

(2) $|\mathrm{Rm}| \leqslant A_{0} Q$, inj $\geqslant i_{0} Q^{-1 / 2}$ and $\left|\nabla^{k} \mathrm{Rm}\right| \leqslant A_{k} Q^{1+k / 2}$ on the union

$$
P_{+}\left(x, t, \sigma Q^{-1 / 2}\right) \cup P_{-}\left(x, t, \sigma Q^{-1 / 2}\right)
$$

of the forward and backward parabolic balls.

Proof. By [44, Lemma 70.1], we have $R\left(x^{\prime}, t^{\prime}\right) \leqslant 8 Q$ for all $\left(x^{\prime}, t^{\prime}\right) \in P_{-}\left(x, t, \eta^{-1} Q^{-1 / 2}\right)$, where $\eta<\infty$ is a universal constant. The same argument works for $P_{+}\left(x, t, \eta^{-1} Q^{-1 / 2}\right)$. Since $R$ is proper on time slices (cf. [44, Lemma 67.9]), it follows that $B\left(x, t, \eta^{-1} Q^{-1 / 2}\right.$ ) has compact closure in its time slice.

If $\mu \leqslant \frac{1}{8}$, then

$$
8 Q=8\left(|R(x, t)|+r(t)^{-2}\right) \leqslant 8\left(|R(x, t)|+r(T)^{-2}\right) \leqslant 8 \mu \varrho(0)^{-2} \leqslant \varrho\left(t^{\prime}\right)^{-2}
$$


for all $t^{\prime} \in\left[t-\eta^{-2} Q^{-1}, t+\eta^{-2} Q^{-1}\right]$. Hence, if $\sigma<\eta^{-1}$ then the forward and backward parabolic balls $P_{ \pm}\left(x, t, \sigma Q^{-1 / 2}\right)$ do not intersect the regions that are affected by the surgery procedure; see Remark A.10.

To show that the balls $P_{ \pm}\left(x, t, \sigma Q^{-1 / 2}\right)$ are unscathed, for an appropriate value of $\sigma$, it remains to show that $P_{-}\left(x, t, \sigma Q^{-1 / 2}\right)$ does not intersect the time-zero slice. We have $t-\sigma^{2} Q^{-1} \geqslant t-\sigma^{2} r(t)^{2}$. Since $t>\frac{1}{100}$ and $r(t)<r(0)<\frac{1}{10}$, if $\sigma<\frac{1}{2}$ then $t-\sigma^{2} Q^{-1} \geqslant \frac{1}{200}$ and the balls $P_{ \pm}\left(x, t, \sigma Q^{-1 / 2}\right)$ are unscathed.

The Hamilton-Ivey estimate of (A.14) gives an explicit upper bound $|\mathrm{Rm}| \leqslant A_{0} Q$ on $P_{ \pm}\left(x, t, \sigma Q^{-1 / 2}\right)$. Using the distance distortion estimates for Ricci flow [44, $\left.\S 27\right]$, there is a universal constant $\alpha>0$ such that, whenever $\left(x^{\prime}, t^{\prime}\right) \in P_{ \pm}\left(x, t, \frac{1}{2} \sigma Q^{-1 / 2}\right)$, we have $P_{-}\left(x^{\prime}, t^{\prime}, \alpha Q^{-1 / 2}\right) \subset P_{+}\left(x, t, \sigma Q^{-1 / 2}\right) \cup P_{-}\left(x, t, \sigma Q^{-1 / 2}\right)$. Then Shi's local derivative estimates [44, Appendix D] give estimates $\left|\nabla^{k} \mathrm{Rm}\right| \leqslant A_{k} Q^{1+k / 2}$ on $P_{ \pm}\left(x, t, \frac{1}{2} \sigma Q^{-1 / 2}\right)$.

Since $t \leqslant T$, we have $\varkappa(t) \geqslant \varkappa(T)$. The $\varkappa$-non-collapsing statement gives an explicit lower bound inj $\geqslant i_{0} Q^{-1 / 2}$ on a slightly smaller parabolic ball, which, after reducing $\sigma$, we can take to be of the form $P_{ \pm}\left(x, t, \sigma Q^{-1 / 2}\right)$.

If $\mathcal{M}$ is a Ricci flow solution and $\gamma:[a, b] \rightarrow \mathcal{M}$ is a time-preserving spacetime curve, then we define length $g_{\mathcal{M}}(\gamma)$ using the spacetime metric $g_{\mathcal{M}}=d t^{2}+g(t)$. The next lemma says that, given a point $\left(x_{0}, t_{0}\right)$ in a $\varkappa$-solution (in the sense of $\S$ A.5), it has a large backward parabolic neighborhood $P$ such that any point $\left(x_{1}, t_{1}\right)$ in $P$ can be connected to $\left(x_{0}, t_{0}\right)$ by a time-preserving curve whose length is controlled by $R\left(x_{0}, t_{0}\right)$, and along which the scalar curvature is controlled by $R\left(x_{0}, t_{0}\right)$.

Lemma 3.2. Given $\varkappa>0$, there exist $A=A(\varkappa)<\infty$ and $C=C(\varkappa)<\infty$ with the following property: if $\mathcal{M}$ is a $\varkappa$-solution and $\left(x_{0}, t_{0}\right) \in \mathcal{M}$, then

(1) there is some $\left(x_{1}, t_{1}\right) \in P_{-}\left(x_{0}, t_{0}, \frac{1}{2} A R\left(x_{0}, t_{0}\right)^{-1 / 2}\right)$ with $R\left(x_{1}, t_{1}\right) \leqslant \frac{1}{3} R\left(x_{0}, t_{0}\right)$;

(2) the scalar curvature on $P_{-}\left(x_{0}, t_{0}, 2 A R\left(x_{0}, t_{0}\right)^{-1 / 2}\right)$ is at most $\frac{1}{2} C R\left(x_{0}, t_{0}\right)$;

(3) given $\left(x_{1}, t_{1}\right) \in P_{-}\left(x_{0}, t_{0}, \frac{1}{2} A R\left(x_{0}, t_{0}\right)^{-1 / 2}\right)$, there is a time-preserving curve

$$
\gamma:\left[t_{1}, t_{0}\right] \longrightarrow P_{-}\left(x_{0}, t_{0}, \frac{3}{4} A R\left(x_{0}, t_{0}\right)^{-1 / 2}\right)
$$

from $\left(x_{1}, t_{1}\right)$ to $\left(x_{0}, t_{0}\right)$ with

$$
\text { length }_{g_{\mathcal{M}}}(\gamma) \leqslant \frac{1}{2} C\left(R\left(x_{0}, t_{0}\right)^{-1 / 2}+R\left(x_{0}, t_{0}\right)^{-1}\right)
$$

Proof. To prove (1), suppose, by way of contradiction, that for each $j \in \mathbb{Z}^{+}$there are a $\varkappa$-solution $\mathcal{M}^{j}$ and some $\left(x_{0}^{j}, t_{0}^{j}\right) \in \mathcal{M}^{j}$ such that $R>\frac{1}{3} R\left(x_{0}^{j}, t_{0}^{j}\right)$ on $P_{-}\left(x_{0}^{j}, t_{0}^{j}, \frac{1}{2} j\right)$. By compactness of the space of normalized pointed $\varkappa$-solutions (see $\S$ A.5), after normalizing so that $R\left(x_{0}^{j}, t_{0}^{j}\right)=1$ and passing to a subsequence, there is a limiting $\varkappa$-solution $\mathcal{M}^{\prime}$, 
defined for $t \leqslant 0$, with $R \geqslant \frac{1}{3}$ everywhere. By the weak maximum principle for complete non-compact manifolds [44, Theorem A.3] and the evolution equation for scalar curvature, there is a universal constant $\Delta>0$ (whose exact value is not important) such that, if $R \geqslant \frac{1}{3}$ on a time- $t$ slice, then there is a singularity by time $t+\Delta$. Applying this with $t=-2 \Delta$ gives a contradiction.

Part (2) of the lemma, for some value of $C$, follows from the compactness of the space of normalized pointed $\varkappa$-solutions.

To prove (3), the curve which starts as a worldline from $\left(x_{1}, t_{1}\right)$ to $\left(x_{1}, t_{0}\right)$, and then moves as a minimal geodesic from $\left(x_{1}, t_{0}\right)$ to $\left(x_{0}, t_{0}\right)$ in the time- $t_{0}$ slice, has $g_{\mathcal{M}^{-}}$ length at most $\frac{1}{2} A R\left(x_{0}, t_{0}\right)^{-1 / 2}+\frac{1}{4} A^{2} R\left(x_{0}, t_{0}\right)^{-1}$. By a slight perturbation to make it time-preserving, we can construct $\gamma$ in $P_{-}\left(x_{0}, t_{0}, \frac{3}{4} A R\left(x_{0}, t_{0}\right)^{-1 / 2}\right)$ with length at most

$$
\frac{1}{2}(A+1) R\left(x_{0}, t_{0}\right)^{-1 / 2}+\frac{1}{4}(A+1)^{2} R\left(x_{0}, t_{0}\right)^{-1} .
$$

After redefining $C$, this proves the lemma.

The next proposition extends the preceding lemma from $\varkappa$-solutions to points in Ricci flows with surgery. Recall that $\varepsilon$ is the global parameter in the definition of Ricci flow with surgery.

Proposition 3.3. There is an $\varepsilon_{0}>0$ such that, if $\varepsilon<\varepsilon_{0}$, then the following holds. Given $T<\infty$, suppose that $\varrho(0) \leqslant r(T) / \sqrt{C}$, where $C$ is the constant from Lemma 3.2. Then, for any $R_{0}<\varrho(0)^{-2} / C$, there are $L=L\left(R_{0}, T\right)<\infty$ and $R_{1}=R_{1}\left(R_{0}, T\right)<\infty$ with the following property. Let $\mathcal{M}$ be a Ricci flow with surgery having normalized initial conditions. Given $\left(x_{0}, t_{0}\right) \in \mathcal{M}$ with $t_{0} \leqslant T$, suppose that $R\left(x_{0}, t_{0}\right) \leqslant R_{0}$. Then, there is a time-preserving curve $\gamma:\left[0, t_{0}\right] \rightarrow \mathcal{M}$ with $\gamma\left(t_{0}\right)=\left(x_{0}, t_{0}\right)$ and $\operatorname{length}_{g_{\mathcal{M}}}(\gamma) \leqslant L$ such that $R(\gamma(t)) \leqslant R_{1}$ for all $t \in\left[0, t_{0}\right]$.

Proof. We begin by noting that we can find $\varepsilon_{0}>0$ such that, if $\varepsilon<\varepsilon_{0}$, then for any $(x, t) \in \mathcal{M}$ with $t \leqslant T$ which is in a canonical neighborhood, Lemma 3.2(2) implies that $R \leqslant C R(x, t)$ on $P_{-}\left(x, t, A R(x, t)^{-1 / 2}\right)$. If in addition $R(x, t) \leqslant \varrho(0)^{-2} / C$, then for any $\left(x^{\prime}, t^{\prime}\right) \in P_{-}\left(x, t, A R(x, t)^{-1 / 2}\right)$ we have

$$
R\left(x^{\prime}, t^{\prime}\right) \leqslant \varrho(0)^{-2} \leqslant \varrho\left(t^{\prime}\right)^{-2} .
$$

Hence, the parabolic neighborhood does not intersect the surgery region.

To prove the proposition, we start with $\left(x_{0}, t_{0}\right)$ and inductively form a sequence of points $\left(x_{i}, t_{i}\right)$ and the curve $\gamma$ as follows, starting with $i=1$.

Step 1. If $R\left(x_{i-1}, t_{i-1}\right) \geqslant r\left(t_{i-1}\right)^{-2}$ then go to Substep A. If $R\left(x_{i-1}, t_{i-1}\right)<r\left(t_{i-1}\right)^{-2}$ then go to Substep B. 
Substep A. Since $R\left(x_{i-1}, t_{i-1}\right) \geqslant r\left(t_{i-1}\right)^{-2}$, the point $\left(x_{i-1}, t_{i-1}\right)$ is in a canonical neighborhood. As will be explained, the backward parabolic ball

$$
P_{-}\left(x_{i-1}, t_{i-1}, A R\left(x_{i-1}, t_{i-1}\right)^{-1 / 2}\right)
$$

does not intersect the surgery region. Applying Lemma 3.2 and taking $\varepsilon_{0}$ small, we can find $\left(x_{i}, t_{i}\right) \in P_{-}\left(x_{i-1}, t_{i-1}, A R\left(x_{i-1}, t_{i-1}\right)^{-1 / 2}\right)$ with $R\left(x_{i}, t_{i}\right) \leqslant \frac{1}{2} R\left(x_{i-1}, t_{i-1}\right)$, and a time-preserving curve

$$
\gamma:\left[t_{i}, t_{i-1}\right] \longrightarrow P_{-}\left(x_{i-1}, t_{i-1}, A R\left(x_{i-1}, t_{i-1}\right)^{-1 / 2}\right)
$$

from $\left(x_{i}, t_{i}\right)$ to $\left(x_{i-1}, t_{i-1}\right)$ whose length is at most

$$
C\left(R\left(x_{i-1}, t_{i-1}\right)^{-1 / 2}+R\left(x_{i-1}, t_{i-1}\right)^{-1}\right),
$$

along which the scalar curvature is at most $C R\left(x_{i-1}, t_{i-1}\right)$. If $t_{i}>0$ then go to Step 2. If $t_{i}=0$ then the process is terminated.

Substep B. Since $R\left(x_{i-1}, t_{i-1}\right)<r\left(t_{i-1}\right)^{-2}$, put $x_{i}=x_{i-1}$ and

$$
t_{i}=\inf \left\{t: R\left(x_{i-1}, s\right) \leqslant r(s)^{-2}\right.
$$

for all $\left.s \in\left[t, t_{i-1}\right]\right\}$. Define $\gamma:\left[t_{i}, t_{i-1}\right] \rightarrow \mathcal{M}$ to be the worldline $\gamma(s)=\left(x_{i-1}, s\right)$.

If $t_{i}>0$, then go to Step 2. (Note that $R\left(x_{i}, t_{i}\right)=r\left(t_{i}\right)^{-2}$.) If $t_{i}=0$, then the process is terminated.

Step 2. Increase $i$ by 1 and go to Step 1 .

To recapitulate the iterative process, if $R_{0}$ is large, then there may initially be a sequence of Substeps A. Since the curvature decreases by a factor of at least 2 for each of these, the number of these initial substeps is bounded above by $\log _{2}\left(R_{0} / r(0)^{-2}\right)$. Thereafter, there is some $\left(x_{i-1}, t_{i-1}\right)$ such that $R\left(x_{i-1}, t_{i-1}\right)<r\left(t_{i-1}\right)^{-2}$. We then go backward in time along a segment of a worldline until we either hit a point $\left(x_{i}, t_{i}\right)$ with $R\left(x_{i}, t_{i}\right)=r\left(t_{i}\right)^{-2}$, or we hit time zero. If we hit $\left(x_{i}, t_{i}\right)$, then we go back to Substep A, which produces a point $\left(x_{i+1}, t_{i+1}\right)$ with at most half as much scalar curvature, etc.

We now check the claim in Substep A that the backward parabolic ball

$$
P_{-}\left(x_{i-1}, t_{i-1}, A R\left(x_{i-1}, t_{i-1}\right)^{-1 / 2}\right)
$$

does not intersect the surgery region. In the initial sequence of Substeps A, we always have $R\left(x_{i-1}, t_{i-1}\right) \leqslant R_{0}<\varrho(0)^{-2} / C$. If we return to Substep A sometime after the initial sequence, we have

$$
R\left(x_{i-1}, t_{i-1}\right)=r\left(t_{i-1}\right)^{-2} \leqslant r(T)^{-2} \leqslant \frac{1}{C} \varrho(0)^{-2} .
$$


Either way, from the first paragraph of the proof, we conclude that

$$
\left.P_{-}\left(x_{i-1}, t_{i-1}, A R\left(x_{i-1}, t_{i-1}\right)^{-1 / 2}\right)^{-1}\right)
$$

does not intersect the surgery region.

We claim that the iterative process terminates. If not, the decreasing sequence $\left(t_{i}\right)$ approaches some $t_{\infty}>0$. For an infinite number of $i$, we must have $R\left(x_{i}, t_{i}\right)=r\left(t_{i}\right)^{-2}$, which converges to $r\left(t_{\infty}\right)^{-2}$. Consider a large $i$ with $R\left(x_{i}, t_{i}\right)=r\left(t_{i}\right)^{-2}$. The result of Substep $\mathrm{A}$ is a point $\left(x_{i+1}, t_{i+1}\right)$ with

$$
R\left(x_{i+1}, t_{i+1}\right) \leqslant \frac{1}{2} R\left(x_{i}, t_{i}\right)=\frac{1}{2} r\left(t_{i}\right)^{-2} \sim \frac{1}{2} r\left(t_{\infty}\right)^{-2} .
$$

If $i$ is large, then this is less than $r\left(t_{i+1}\right)^{-2} \sim r\left(t_{\infty}\right)^{-2}$. Hence, one goes to Substep B to find $\left(x_{i+1}, t_{i+2}\right)$ with $R\left(x_{i+1}, t_{i+2}\right)=r\left(t_{i+2}\right)^{-2}$. However, there is a double-sided bound on

$$
\frac{\partial R}{\partial t}\left(x_{i+1}, t\right) \text { for } t \in\left[t_{i+2}, t_{i+1}\right]
$$

coming from the curvature bound on a backward parabolic ball in [44, Lemma 70.1] and Shi's local estimates [44, Appendix D]. This bound implies that the amount of backward time required to go from a point with scalar curvature $R\left(x_{i+1}, t_{i+1}\right) \leqslant \frac{1}{2} r\left(t_{i}\right)^{-2} \sim \frac{1}{2} r\left(t_{\infty}\right)^{-2}$ to a point with scalar curvature $R\left(x_{i+1}, t_{i+2}\right)=r\left(t_{i+2}\right)^{-2} \sim r\left(t_{\infty}\right)^{-2}$ satisfies

$$
t_{i+1}-t_{i+2} \geqslant \text { const. } r\left(t_{\infty}\right)^{2} \text {. }
$$

This contradicts the fact that $\lim _{i \rightarrow \infty} t_{i}=t_{\infty}$.

We note that the preceding argument can be made effective. This gives a upper bound $N$ on the number of points $\left(x_{i}, t_{i}\right)$ of the form $N=N\left(R_{0}, T\right)$. We now estimate the length of $\gamma$. The contribution to the length from segments arising from Substep B is at most $T$. The contribution from segments arising from Substep A is bounded above by $N C\left(r(0)+r(0)^{2}\right)$.

It remains to estimate the scalar curvature along $\gamma$. Along a portion of $\gamma$ arising from Substep A, the scalar curvature is bounded above by $C R\left(x_{i-1}, t_{i-1}\right) \leqslant C \max \left(R_{0}, r(T)^{-2}\right)$. Along a portion of $\gamma$ arising from Substep B, the scalar curvature is bounded above by $r(T)^{-2}$. Thus we can take $R_{1}=(C+1)\left(R_{0}+r(T)^{-2}\right)$. This proves the proposition.

Finally, we give an estimate on the volume of the high-curvature region in a Ricci flow with surgery. This estimate will be used to prove the volume convergence statement in Theorem 1.3. 
Proposition 3.4. Given $T<\infty$, there are functions $\varepsilon_{1,2}^{T}:[0, \infty) \rightarrow[0, \infty)$, with

$$
\lim _{\bar{R} \rightarrow \infty} \varepsilon_{1}^{T}(\bar{R})=0 \quad \text { and } \quad \lim _{\delta \rightarrow 0} \varepsilon_{2}^{T}(\delta)=0
$$

having the following property. Let $\mathcal{M}$ be a Ricci flow with surgery, with normalized initial condition. Let $\mathcal{V}(0)$ denote its initial volume. Given $\bar{R}>r(T)^{-2}$, if $t$ is not a surgery time, then let $\mathcal{V} \geqslant \bar{R}(t)$ be the volume of the corresponding superlevel set of $R$ in $\mathcal{M}_{t}$. If $t$ is a surgery time, let $\mathcal{V} \geqslant \bar{R}(t)$ be the volume of the corresponding superlevel set of $R$ in the post-surgery manifold $\mathcal{M}_{t}^{+}$. Then, if $t \in[0, T]$, we have

$$
\mathcal{V}^{\geqslant} \bar{R}(t) \leqslant\left(\varepsilon_{1}^{T}(\bar{R})+\varepsilon_{2}^{T}(\delta(0))\right) \mathcal{V}(0) .
$$

Proof. Suppose first that $\mathcal{M}$ is a smooth Ricci flow. Given $t \in[0, T]$, let $i_{t}: \mathcal{M}_{0} \rightarrow \mathcal{M}_{t}$ be the identity map. For $x \in \mathcal{M}_{0}$, put

$$
J_{t}(x)=\frac{i_{t}^{*} d \operatorname{vol}_{g(t)}}{d \operatorname{vol}_{g(0)}}(x) .
$$

Let $\gamma_{x}:[0, t] \rightarrow \mathcal{M}$ be the worldline of $x$. From the Ricci flow equation,

$$
J_{t}(x)=e^{-\int_{0}^{t} R\left(\gamma_{x}(s)\right) d s} .
$$

Suppose that $m \in \mathcal{M}_{t}$ satisfies $R(m) \geqslant \bar{R}$. Then $R(m)>r(t)^{-2}$. Let $x \in \mathcal{M}_{0}$ be the point where the worldline of $m$ hits $\mathcal{M}_{0}$. From (A.8) we have

$$
\frac{d R\left(\gamma_{x}(s)\right)}{d s} \leqslant \eta R\left(\gamma_{x}(s)\right)^{2}
$$

as long as $R\left(\gamma_{x}(s)\right) \geqslant r(s)^{-2}$. Let $t_{1}$ be the smallest number such that $R\left(\gamma_{x}(s)\right) \geqslant r(s)^{-2}$ for all $s \in\left[t_{1}, t\right]$. Since $r(0)<\frac{1}{10}$, the normalized initial conditions imply that $t_{1}>0$. From $(3.5)$, if $s \in\left[t_{1}, t\right]$ then

$$
R\left(\gamma_{x}(s)\right) \geqslant \frac{1}{R(m)^{-1}+\eta(t-s)} .
$$

In particular,

$$
r\left(t_{1}\right)^{-2}=R\left(t_{1}\right) \geqslant \frac{1}{R(m)^{-1}+\eta\left(t-t_{1}\right)} .
$$

From (3.6),

$$
\int_{t_{1}}^{t} R\left(\gamma_{x}(s)\right) d s \geqslant-\frac{1}{\eta} \log \frac{R(m)^{-1}}{R(m)^{-1}+\eta\left(t-t_{1}\right)}
$$

Hence,

$$
e^{-\int_{t_{1}}^{t} R\left(\gamma_{x}(s)\right) d s} \leqslant\left(\frac{R(m)^{-1}}{R(m)^{-1}+\eta\left(t-t_{1}\right)}\right)^{1 / \eta} \leqslant\left(R(m) r\left(t_{1}\right)^{2}\right)^{-1 / \eta} \leqslant\left(R(m) r(T)^{2}\right)^{-1 / \eta}
$$


On the other hand, for all $s \geqslant 0$, equation (A.11) gives

$$
R\left(\gamma_{x}(s)\right) \geqslant-\frac{3}{1+2 s}
$$

So

$$
e^{-\int_{0}^{t_{1}} R\left(\gamma_{x}(s)\right) d s} \leqslant\left(1+2 t_{1}\right)^{3 / 2}
$$

Thus,

$$
J_{t}(x) \leqslant(1+2 T)^{3 / 2}\left(R(m) r(T)^{2}\right)^{-1 / \eta} .
$$

Integrating over such $x \in \mathcal{M}_{0}$, arising from worldlines emanating from

$$
\left\{m \in \mathcal{M}_{t}: R(m) \geqslant \bar{R}\right\},
$$

we conclude that

$$
\mathcal{V}^{\geqslant} \bar{R}(t) \leqslant(1+2 T)^{3 / 2}\left(\bar{R} r(T)^{2}\right)^{-1 / \eta} \mathcal{V}(0),
$$

so the conclusion of the proposition holds in this case with

$$
\varepsilon_{1}^{T}(\bar{R})=(1+2 T)^{3 / 2}\left(\bar{R} r(T)^{2}\right)^{-1 / \eta}
$$

Now suppose that $\mathcal{M}$ has surgeries. For simplicity of notation, we assume that $t$ is not a surgery time; otherwise, we replace $\mathcal{M}_{t}$ by $\mathcal{M}_{t}^{+}$. We can first apply the preceding argument to the subset of (3.7) consisting of points whose worldline goes back to $\mathcal{M}_{0}$. The conclusion is that the volume of this subset is bounded above by $\varepsilon_{1}^{T}(\bar{R}) \mathcal{V}(0)$, where $\varepsilon_{1}^{T}(\bar{R})$ is the same as in (3.8). Now consider the subset of (3.7) consisting of points whose worldline does not go back to $\mathcal{M}_{0}$. We can cover such points by the forward images of surgery caps (or rather the subsets thereof which go forward to time $t$ ), for surgeries that occur at times $t_{\alpha} \leqslant t$. Let $\mathcal{V}_{t_{\alpha}}^{\text {cap }}$ be the total volume of the surgery caps for surgeries that occur at time $t_{\alpha}$. Let $\mathcal{V}_{t_{\alpha}}^{\text {remove }}$ be the total volume that is removed at time $t_{\alpha}$ by the surgery process. From the nature of the surgery process $[44, \S 72]$, there is an increasing function $\delta^{\prime}:(0, \infty) \rightarrow(0, \infty)$, with $\lim _{\delta \rightarrow 0} \delta^{\prime}(\delta)=0$, such that

$$
\frac{\mathcal{V}_{t_{\alpha}}^{\text {cap }}}{\mathcal{V}_{t_{\alpha}}^{\text {remove }}} \leqslant \delta^{\prime}(\delta(0))
$$

This is essentially because the surgery procedure removes a long capped tube, whose length (relative to $h(t)$ ) is large if $\delta(t)$ is small, and replaces it by a hemispherical cap.

On the other hand, using (A.12),

$$
\sum_{t_{\alpha} \leqslant t} \mathcal{V}_{t_{\alpha}}^{\text {remove }} \leqslant(1+2 T)^{3 / 2} \mathcal{V}(0)
$$


since surgeries up to time $t$ cannot remove more volume than was initially present or generated by the Ricci flow. The time- $t$ volume coming from forward worldlines of surgery caps is at most

$$
(1+2 T)^{3 / 2} \sum_{t_{\alpha} \leqslant t} \mathcal{V}_{t_{\alpha}}^{\text {cap }}
$$

Hence, the proposition is true if we take

$$
\varepsilon_{2}^{T}(\delta)=(1+2 T)^{3} \delta^{\prime}(\delta)
$$

\section{The main convergence result}

In this section, we prove Theorem 4.1 except for the statement about the continuity of $\mathcal{V}_{\infty}$, which will be proved in Corollary 7.7 .

The convergence assertion in Theorem 4.1 involves a sequence $\left\{\mathcal{M}^{j}\right\}_{j=1}^{\infty}$ of Ricci flows with surgery, where the functions $r$ and $\varkappa$ are fixed, but $\delta_{j} \rightarrow 0$; hence, $\varrho_{j}$ and $h_{j}$ also go to zero. We will conflate these Ricci flows with surgery with their associated Ricci flow spacetimes; see $\S$ A.9.

THEOREM 4.1. Let $\left\{\mathcal{M}^{j}\right\}_{j=1}^{\infty}$ be a sequence of Ricci flows with surgery with normalized initial conditions such that the following conditions are satisfied:

- the time-zero slices $\left\{\mathcal{M}_{0}^{j}\right\}$ are compact manifolds that lie in a compact family in the smooth topology;

- $\lim _{j \rightarrow \infty} \delta_{j}(0)=0$.

Then, after passing to a subsequence, there is a singular Ricci flow

$$
\left(\mathcal{M}^{\infty}, \mathfrak{t}_{\infty}, \partial_{\mathfrak{t}_{\infty}}, g_{\infty}\right)
$$

and a sequence of diffeomorphisms

$$
\left\{\mathcal{M}^{j} \supset U_{j} \stackrel{\Phi^{j}}{\longrightarrow} V_{j} \subset \mathcal{M}^{\infty}\right\}
$$

such that the following conditions are satisfied:

(1) $U_{j} \subset \mathcal{M}^{j}$ and $V_{j} \subset \mathcal{M}^{\infty}$ are open subsets;

(2) given $\bar{t}>0$ and $\bar{R}<\infty$, we have

$$
\begin{aligned}
& U_{j} \supset\left\{m \in \mathcal{M}^{j}: \mathfrak{t}_{j}(m) \leqslant \bar{t} \text { and } R_{j}(m) \leqslant \bar{R}\right\}, \\
& V_{j} \supset\left\{m \in \mathcal{M}^{\infty}: \mathfrak{t}_{\infty}(m) \leqslant \bar{t} \text { and } R_{\infty}(m) \leqslant \bar{R}\right\}
\end{aligned}
$$

for all sufficiently large $j$; 
(3) $\Phi^{j}$ is time preserving, and the sequences $\left\{\Phi_{*}^{j} \partial_{\mathfrak{t}_{j}}\right\}_{j=1}^{\infty}$ and $\left\{\Phi_{*}^{j} g_{j}\right\}_{j=1}^{\infty}$ converge smoothly on compact subsets of $\mathcal{M}^{\infty}$ to $\partial_{t_{\infty}}$ and $g_{\infty}$, respectively (note that, by (4.3), any compact set $K \subset \mathcal{M}^{\infty}$ will lie in the interior of $V_{j}$, for all sufficiently large $j$ );

(4) for every $t \geqslant 0$, we have

$$
\inf _{\mathcal{M}_{t}^{\infty}} R \geqslant-\frac{3}{1+2 t}
$$

and

$$
\max \left(\mathcal{V}_{j}(t), \mathcal{V}_{\infty}(t)\right) \leqslant V_{0}(1+2 t)^{3 / 2}
$$

where $\mathcal{V}_{j}(t)=\operatorname{vol}\left(\mathcal{M}_{t}^{j}\right), \mathcal{V}_{\infty}(t)=\operatorname{vol}\left(\mathcal{M}_{t}^{\infty}\right)$ and $V_{0}=\sup _{j} \mathcal{V}_{j}(0)<\infty$;

(5) $\Phi^{j}$ is asymptotically volume preserving: if $\mathcal{V}_{j}, \mathcal{V}_{\infty}:[0, \infty) \rightarrow[0, \infty)$ denote the respective volume functions $\mathcal{V}_{j}(t)=\operatorname{vol}\left(\mathcal{M}_{t}^{j}\right)$ and $\mathcal{V}_{\infty}(t)=\operatorname{vol}\left(\mathcal{M}_{t}^{\infty}\right)$, then $\lim _{j \rightarrow \infty} \mathcal{V}_{j}=\mathcal{V}_{\infty}$ uniformly on compact subsets of $[0, \infty)$.

Proof. Because of the normalized initial conditions, the Ricci flow solution $g_{j}$ is smooth on the time interval $\left[0, \frac{1}{100}\right]$. As a technical device, we first extend $g_{j}$ backward in time to a family of metrics $g_{j}(t)$ which is smooth for $t \in\left(-\infty, \frac{1}{100}\right]$. To do this, as in [41, proof of Theorem 1.2.6], there is an explicit smooth extension $h_{j}$ of $g_{j}(t)-g_{j}(0)$ to the time interval $t \in\left(-\infty, \frac{1}{100}\right]$, with values in smooth covariant 2 -tensor fields. The extension on $(-\infty, 0]$ depends on the time-derivatives of $g_{j}(t)-g_{j}(0)$ at $t=0$ which, in turn, can be expressed in terms of $g_{j}(0)$ by means of repeated differentiation of the Ricci flow equation. Let $\phi:[0, \infty) \rightarrow[0,1]$ be a fixed non-increasing smooth function with $\left.\phi\right|_{[0,1]}=1$ and $\left.\phi\right|_{[2, \infty)}=0$. Given $\varepsilon>0$, for $t<0$ put

$$
g_{j}(t)=g_{j}(0)+\phi\left(-\frac{t}{\varepsilon}\right) h_{j}(t)
$$

Using the precompactness of the space of initial conditions, we can choose $\varepsilon$ small enough so that $g_{j}(t)$ is a Riemannian metric for all $j$ and all $t \leqslant 0$. Then

(1) $g_{j}(t)$ is smooth in $t \in\left(-\infty, \frac{1}{100}\right]$;

(2) $g_{j}(t)$ is constant in $t$ for $t \leqslant-2 \varepsilon$;

(3) for $t \leqslant 0, g_{j}(t)$ has uniformly bounded curvature and curvature derivatives, independent of $j$.

Let $g_{\mathcal{M}^{j}}$ be the spacetime Riemannian metric on $\mathcal{M}^{j}$ (see Definition 1.7). Choose a basepoint $\star_{j} \in \mathcal{M}_{0}^{j}$.

After passing to a subsequence, we may assume that, for all $j$,

$$
\varrho_{j}(0) \leqslant \sqrt{\frac{\mu}{j+r(j)^{-2}}}
$$


where $\mu=\mu(j)$ is the parameter of Lemma 3.1. Put $\psi_{j}(x, t)=R_{j}(x, t)^{2}+t^{2}$, where $R_{j}$ is the scalar curvature function of the Ricci flow metric $g_{j}$. Put $A_{j}=j^{2}$. If $(x, t) \in \psi_{j}^{-1}\left(\left[0, A_{j}\right)\right)$, then $\left|R_{j}(x, t)\right| \leqslant j$ and $|t| \leqslant j$. In particular,

$$
\left|R_{j}(x, t)\right| \leqslant j \leqslant \mu \varrho_{j}(0)^{-2}-r(j)^{-2} \leqslant \varrho_{j}(0)^{-2} \leqslant \varrho_{j}(t)^{-2},
$$

so $(x, t)$ avoids the surgery region.

We claim that there are functions $\mathfrak{r}$ and $C_{k}$ such that Definition 2.12 holds for all $j$. Suppose that $\psi_{j}(x, t) \leqslant A_{j}$. When $t \leqslant 0$ there is nothing to prove, so we assume that $t \geqslant 0$. If $t \in\left[0, \frac{1}{100}\right]$, then the normalized initial conditions give uniform control on $g_{j}(t)$. If $t \geqslant \frac{1}{100}$, then

$$
\operatorname{tr}(t)^{-2} \geqslant \frac{1}{100} r(0)^{-2}>1>\sigma^{2},
$$

where $\sigma=\sigma(j)$ is the parameter from Lemma 3.1, and we use the assumption that $r(0)<\frac{1}{10}$ from the beginning of $\S 3$. Then,

$$
t>\sigma^{2} r(t)^{2} \geqslant \sigma^{2} \frac{1}{|R(x, t)|+r(t)^{-2}}=\sigma^{2} Q^{-1},
$$

so the parabolic ball $P_{-}\left(x, t, \sigma Q^{-1 / 2}\right)$ of Lemma 3.1 does not intersect the initial time slice. As $\left|R_{j}(x, t)\right| \leqslant \mu \varrho_{j}(0)^{-2}-r(j)^{-2}$ from (4.5), we can apply Lemma 3.1 to show that $\left(\mathcal{M}^{j}, g_{\mathcal{M}^{j}}, \psi_{j}\right)$ is $\left(\psi_{j}, A_{j}\right)$-controlled in the sense of Definition 2.1. Note that Lemma 3.1 gives bounds on the spatial and time derivatives of the curvature tensor of $g_{j}(t)$, which implies bounds on the derivatives of the curvature tensor of the spacetime metric $g_{\mathcal{M}_{j}}$.

Finally, the function $\mathfrak{t}_{j}$ and the vector field $\partial_{\mathfrak{t}_{j}}$, along with their covariant derivatives, are trivially bounded in terms of $g_{\mathcal{M}^{j}}$.

After passing to a subsequence, we may assume that the number $N$ of connected components of the initial time slice $\mathcal{M}_{0}^{j}$ is independent of $j$. Then, the theorem follows from the special case when the initial times slices are connected, since we may apply it to the components separately. Therefore, we are reduced to proving the theorem under the assumption that $\mathcal{M}_{0}^{j}$ is connected.

After passing to a subsequence, Theorem 2.13 now gives a pointed $\left(\psi_{\infty}, \infty\right)$-controlled tuple

$$
\left(\mathcal{M}^{\infty}, g_{\mathcal{M}^{\infty}}, \mathfrak{t}_{\infty},\left(\partial_{\mathfrak{t}}\right)_{\infty}, \star_{\infty}, \psi_{\infty}\right)
$$

satisfying conditions (1)-(4) of Theorem 2.13. We truncate $\mathcal{M}^{\infty}$ to the subset $\mathfrak{t}_{\infty}^{-1}([0, \infty))$. We now verify the claims of Theorem 4.1 .

Part (1) of Theorem 4.1 follows from the statement of Theorem 2.13. Given $\bar{t}>0$ and $\bar{R}<\infty$, Proposition 3.3 implies that there are $r=r(\bar{R}, \bar{t})<\infty$ and $A=A(\bar{R}, \bar{t})<\infty$ such that the set $\left\{m \in \mathcal{M}_{\leqslant \bar{t}}^{j}: R(m) \leqslant \bar{R}\right\}$ is contained in the metric ball $B\left(\star_{j}, r\right)$ in the 
Riemannian manifold $\left(\psi_{j}^{-1}([0, A)), g_{\mathcal{M}^{j}}\right)$. Hence, by the definition of $\psi_{j}$ and part (1) of Theorem 2.13, we get (4.2).

Pick $m_{\infty} \in \mathcal{M}^{\infty}$. Put $t_{\infty}=\mathfrak{t}\left(m_{\infty}\right)$. By Theorem 2.13 (4), we know that $m_{\infty}$ belongs to $V_{j}$ for large $j$. Now, Theorem $2.13(3)$ and the definition of $\psi_{j}$ imply that

$$
\psi_{\infty}\left(m_{\infty}\right)=R\left(m_{\infty}\right)^{2}+t_{\infty}^{2} .
$$

If $j$ is large, then it makes sense to define $\left(x_{j}, t_{\infty}\right)=\left(\Phi^{j}\right)^{-1}\left(m_{\infty}\right) \in \mathcal{M}^{j}$. Then, for $j$ large, $R\left(x_{j}, t_{\infty}\right)<R\left(m_{\infty}\right)+1$ and so, by Proposition 3.3, there is a time-preserving curve $\gamma_{j}:\left[0, t_{\infty}\right] \rightarrow \mathcal{M}^{j}$ such that

$$
\max \left(\text { length }_{g_{\mathcal{M}^{j}}}\left(\gamma_{j}\right), \max _{t \in\left[0, t_{\infty}\right]} R\left(\gamma_{j}(t)\right)\right)<C=C\left(R\left(m_{\infty}\right), t_{\infty}\right) .
$$

By Theorem 2.13(1), we know that $\operatorname{Im}\left(\gamma_{j}\right) \subset U_{j}$ for large $j$. By Theorem 2.13(3), for large $j$ the map $\Phi^{j}$ is an almost-isometry. Hence, there are $r=r\left(R\left(m_{\infty}\right), t_{\infty}\right)<\infty$ and $A=A\left(R\left(m_{\infty}\right), t_{\infty}\right)<\infty$ such that $m_{\infty}$ is contained in the metric ball $B\left(\star_{\infty}, r\right)$ in the Riemannian manifold $\left(\left(\psi_{\infty}\right)^{-1}([0, A)), g_{\mathcal{M}^{\infty}}\right)$. Combining this with (4.6) and Theorem 2.13 (1) yields (4.3). This proves Theorem $4.1(2)$.

Theorem 4.1(3) now follows from Theorem 4.1(2) and from parts (2) and (3) of Theorem 2.13.

Equation (4.4) follows from (A.11) and the smooth approximation in part (3) of Theorem 4.1. Let $\mathcal{V}_{j}^{<\bar{R}}(t)$ be the volume of the $\bar{R}$-sublevel set for the scalar curvature function on $\mathcal{M}_{t}^{j}$. (If $t$ is a surgery time, we replace $\mathcal{M}_{t}$ by $\mathcal{M}_{t}^{+}$.) Let $\mathcal{V}_{\infty}^{<\bar{R}}(t)$ be the volume of the $\bar{R}$-sublevel set for the scalar curvature function on $\mathcal{M}_{t}^{\infty}$. Then,

$$
\mathcal{V}_{\infty}(t)=\lim _{\bar{R} \rightarrow \infty} \mathcal{V}_{\infty}^{<\bar{R}}(t)=\lim _{\bar{R} \rightarrow \infty} \lim _{j \rightarrow \infty} \mathcal{V}_{j}^{<\bar{R}}(t) \leqslant \limsup _{j \rightarrow \infty} \mathcal{V}_{j}(t)
$$

Theorem 4.1 (4) now follows from combining this with (A.12).

Next, we verify the volume convergence assertion (5). Let $\|\cdot\|_{T}$ denote the sup norm on $L^{\infty}([0, T])$. By parts (2) and (3) of Theorem 4.1, there is some $\varepsilon_{3}^{T}(j, \bar{R})>0$, with $\lim _{j \rightarrow \infty} \varepsilon_{3}^{T}(j, \bar{R})=0$, such that

$$
\left\|\mathcal{V}_{\infty}^{<\bar{R}}-\mathcal{V}_{j}^{<\bar{R}}\right\|_{T} \leqslant \varepsilon_{3}^{T}(j, \bar{R}) .
$$

Also, if $\bar{S}>\bar{R}$, then

$$
\left\|\mathcal{V}_{\infty}^{<\bar{S}}-\mathcal{V}_{\infty}^{<\bar{R}}\right\|_{T}=\lim _{j \rightarrow \infty}\left\|\mathcal{V}_{j}^{<\bar{S}}-\mathcal{V}_{j}^{<\bar{R}}\right\|_{T} \leqslant \limsup _{j \rightarrow \infty}\left\|\mathcal{V}_{j}-\mathcal{V}_{j}^{<\bar{R}}\right\|_{T}
$$

Proposition 3.4 implies that

$$
\left\|\mathcal{V}_{j}-\mathcal{V}_{j}^{<\bar{R}}\right\|_{T} \leqslant\left(\varepsilon_{1}^{T}(\bar{R})+\varepsilon_{2}^{T}\left(\delta_{j}(0)\right)\right) V_{0} .
$$


Combining (4.8) and (4.9), and taking $\bar{S} \rightarrow \infty$, gives

$$
\left\|\mathcal{V}_{\infty}-\mathcal{V}_{\infty}^{<\bar{R}}\right\|_{T} \leqslant \varepsilon_{1}^{T}(\bar{R}) V_{0}
$$

Combining (4.7), (4.9) and (4.10) yields

$$
\left\|\mathcal{V}_{j}-\mathcal{V}_{\infty}\right\|_{T} \leqslant\left(2 \varepsilon_{1}^{T}(\bar{R})+\varepsilon_{2}^{T}\left(\delta_{j}(0)\right)\right) V_{0}+\varepsilon_{3}^{T}(j, \bar{R})
$$

Given $\sigma>0$, we can choose $\bar{R}<\infty$ so that $2 \varepsilon_{1}^{T}(\bar{R}) V_{0}<\frac{1}{2} \sigma$. Given this value of $\bar{R}$, we can choose $J$ so that, if $j \geqslant J$, then

$$
\varepsilon_{2}^{T}\left(\delta_{j}(0)\right) V_{0}+\varepsilon_{3}^{T}(j, \bar{R})<\frac{1}{2} \sigma
$$

Hence, if $j \geqslant J$, then $\left\|\mathcal{V}_{j}-\mathcal{V}_{\infty}\right\|_{T}<\sigma$. This shows that $\lim _{j \rightarrow \infty} \mathcal{V}_{j}=\mathcal{V}_{\infty}$, uniformly on compact subsets of $[0, \infty)$, and proves Theorem $4.1(5)$.

Finally, we check that $\mathcal{M}^{\infty}$ is a singular Ricci flow in the sense of Definition 1.4. Using Theorem 4.1(3), one can pass the Hamilton-Ivey pinching condition, canonical neighborhoods, and the non-collapsing condition from the $\mathcal{M}^{j}$ 's to $\mathcal{M}^{\infty}$, and so parts (b) and (c) of Definition 1.4 hold.

We now verify Definition 1.4(a). We start with a statement about parabolic neighborhoods in $\mathcal{M}^{\infty}$. Given $T>0$ and $\bar{R}<\infty$, suppose that $m_{\infty} \in \mathcal{M}^{\infty}$ has $\mathfrak{t}\left(m_{\infty}\right) \leqslant T$ and $R\left(m_{\infty}\right) \leqslant \bar{R}$. For large $j$, put $\widehat{m}_{j}=\left(\Phi^{j}\right)^{-1}\left(m_{\infty}\right) \in \mathcal{M}^{j}$. Lemma 3.1 supplies parabolic regions centered at the $\widehat{m}_{j}$ 's which pass to $\mathcal{M}^{\infty}$. Hence, for some $r=r(T, \bar{R})>0$, the forward and backward parabolic regions $P_{+}\left(m_{\infty}, r\right)$ and $P_{-}\left(m_{\infty}, r\right)$ are unscathed. There is some $K=K(T, \bar{R})<\infty$ such that, when equipped with the spacetime metric $g_{\mathcal{M}^{\infty}}$, the union $P_{+}\left(m_{\infty}, r\right) \cup P_{-}\left(m_{\infty}, r\right)$ is $K$-bilipschitz homeomorphic to a Euclidean parabolic region.

From (4.4), we know that $R$ is bounded below on $\mathcal{M}_{[0, T]}^{\infty}$. In order to show that $R$ is proper on $\mathcal{M}_{[0, T]}^{\infty}$, we need to show that any sequence in a sublevel set of $R$ has a convergent subsequence. Suppose that $\left\{m_{k}\right\}_{k=1}^{\infty} \subset \mathcal{M}^{\infty}$ is a sequence with $\mathfrak{t}\left(m_{k}\right) \leqslant T$ and $R\left(m_{k}\right) \leqslant \bar{R}$. After passing to a subsequence, we may assume that $\mathfrak{t}\left(m_{k}\right) \rightarrow t_{\infty} \in[0, \infty)$. Then, the regions $P\left(m_{k}, \frac{1}{100} r, r^{2}\right) \cup P\left(m_{k}, \frac{1}{100} r,-r^{2}\right)$ will intersect the time slice $\mathcal{M}_{t_{\infty}}$ in regions whose volume is bounded below by a constant times $r^{3}$. By the volume bound in Theorem 4.1 (4), only finitely many of these can be disjoint. Therefore, after passing to a subsequence, $\left\{m_{k}\right\}_{k=1}^{\infty}$ is contained in $P_{+}\left(m_{\ell}, \frac{1}{2} r\right) \cup P_{-}\left(m_{\ell}, \frac{1}{2} r\right)$ for some $\ell$. As $P_{+}\left(m_{\ell}, \frac{1}{2} r\right) \cup P_{-}\left(m_{\ell}, \frac{1}{2} r\right)$ has compact closure in $P_{+}\left(m_{\ell}, r\right) \cup P_{-}\left(m_{\ell}, r\right)$, a subsequence of $\left\{m_{k}\right\}_{k=1}^{\infty}$ converges. This verifies Definition $1.4(\mathrm{a})$. 


\section{Part II}

\section{Basic properties of singular Ricci flows}

In this section we prove some initial structural properties of Ricci flow spacetimes and singular Ricci flows. In $\S 5.1$ we justify the maximum principle on a Ricci flow spacetime and apply it to get a lower scalar curvature bound. In $\S 5.2$ we prove some results about volume evolution for Ricci flow spacetimes which satisfy certain assumptions, that are satisfied in particular for singular Ricci flows. The main result in $\S 5.3$ says that, if $\mathcal{M}$ is a singular Ricci flow and $\gamma_{0}, \gamma_{1}:\left[t_{0}, t_{1}\right] \rightarrow \mathcal{M}$ are two time-preserving curves such that $\gamma_{0}\left(t_{1}\right)$ and $\gamma_{1}\left(t_{1}\right)$ are in the same connected component of $\mathcal{M}_{t_{1}}$, then $\gamma_{0}(t)$ and $\gamma_{1}(t)$ are in the same connected component of $\mathcal{M}_{t}$ for all $t \in\left[t_{0}, t_{1}\right]$.

We recall the notion of a Ricci flow spacetime from Definition 1.2. In this section, we will consider it to only be defined for non-negative time, i.e. $\mathfrak{t}$ takes value in $[0, \infty)$. We also recall the metrics $g_{\mathcal{M}}$ and $g_{\mathcal{M}}^{\mathrm{qp}}$ from Definition 1.7. Let $n+1$ be the dimension of $\mathcal{M}$. Our notation is the following:

- $\mathcal{M}_{t}=\mathfrak{t}^{-1}(t)$;

- $\mathcal{M}_{[a, b]}=\mathfrak{t}^{-1}([a, b])$;

- $\mathcal{M}_{\leqslant T}=\mathfrak{t}^{-1}([0, T])$.

\subsection{Maximum principle and scalar curvature}

In this subsection we prove a maximum principle on Ricci flow spacetimes and apply it to get a lower bound on scalar curvature.

Lemma 5.1. Let $\mathcal{M}$ be a Ricci flow spacetime. Given $T \in(0, \infty)$, let $X$ be a smooth vector field on $\mathcal{M}_{\leqslant T}$ with $X \mathfrak{t}=0$. Given a smooth function $F: \mathbb{R} \times[0, T] \rightarrow \mathbb{R}$, suppose that $u \in C^{\infty}\left(\mathcal{M}_{\leqslant T}\right)$ is a proper function, bounded above, which satisfies

$$
\partial_{\mathfrak{t}} u \leqslant \triangle_{g(\mathfrak{t})} u+X u+F(u, \mathfrak{t})
$$

Suppose further that $\phi:[0, T] \rightarrow \mathbb{R}$ satifies

$$
\partial_{\mathfrak{t}} \phi=F(\phi(\mathfrak{t}), \mathfrak{t})
$$

with initial condition $\phi(0)=\alpha \in \mathbb{R}$. If $u \leqslant \alpha$ on $\mathcal{M}_{0}$, then $u \leqslant \phi \circ$ on $\mathcal{M}_{\leqslant T}$.

Proof. As in [73, proof of Theorem 3.1.1], for $\varepsilon>0$, we consider the ordinary differential equation (ODE)

$$
\partial_{t} \phi_{\varepsilon}=F\left(\phi_{\varepsilon}(t), t\right)+\varepsilon
$$


with initial condition $\phi_{\varepsilon}(0)=\alpha+\varepsilon$. It suffices to show that for all small $\varepsilon$ we have $u<\phi_{\varepsilon}$ on $\mathcal{M}_{\leqslant}$.

If not, then we can find some $\varepsilon>0$ such that the property $u<\phi_{\varepsilon}$ fails on $\mathcal{M}_{\leqslant T}$. As $u$ is proper and bounded above, there is a first time $t_{0}$ such that the property fails on $\mathcal{M}_{t_{0}}$, and an $m \in \mathcal{M}_{t_{0}}$ such that $u(m)=\phi_{\varepsilon}\left(t_{0}\right)$. The rest of the argument is the same as in [73, proof of Theorem 3.1.1].

Lemma 5.2. Let $\mathcal{M}$ be a Ricci flow spacetime. Suppose that for each $T \geqslant 0$ the scalar curvature $R$ is proper and bounded below on $\mathcal{M}_{\leqslant T}$. Suppose that the initial scalar curvature is bounded below by $-C$, for some $C \geqslant 0$. Then

$$
R(m) \geqslant-\frac{C}{1+2 C \mathfrak{t}(m) / n}
$$

Proof. Since $R$ is proper on $\mathcal{M}_{\leqslant T}$, we may apply Lemma 5.1 to the evolution equation for $-R$ and follow the standard proof to get (5.3).

\subsection{Volume}

In this subsection we first justify a Fubini-type statement for Ricci flow spacetimes. Then we show that certain standard volume estimates for smooth Ricci flows extend to the setting of Ricci flow spacetimes under two assumptions: first that the quasi-parabolic metric is complete along worldlines that do not terminate at the time-zero slice, and second that in any time slice almost all points have worldlines that extend backward to time zero.

The Fubini-type statement is the following.

Lemma 5.4. Let $\mathcal{M}$ be a Ricci flow spacetime. Given $0<t_{1}<t_{2}<\infty$, suppose that $F: \mathcal{M}_{\left[t_{1}, t_{2}\right]} \rightarrow \mathbb{R}$ is measurable and bounded below. Suppose that $\mathcal{M}_{\left[t_{1}, t_{2}\right]}$ has finite volume with respect to $g_{\mathcal{M}}$. Then,

$$
\int_{\mathcal{M}_{\left[t_{1}, t_{2}\right]}} F d \operatorname{vol}_{g_{\mathcal{M}}}=\int_{t_{1}}^{t_{2}} \int_{M_{t}} F d \operatorname{vol}_{g(t)} d t .
$$

Proof. As $F$ is bounded below on $\mathcal{M}_{\left[t_{1}, t_{2}\right]}$, for the purposes of the proof we can add a constant to $F$ and assume that it is positive. Given $m \in \mathcal{M}_{\left[t_{1}, t_{2}\right]}$, there is a timepreserving embedding $e:(a, b) \times X \rightarrow \mathcal{M}$ with $e_{*}\left(\partial_{s}\right)=\partial_{\mathfrak{t}}$ (where $s \in(a, b)$ ) whose image is a neighborhood of $m$. We can cover $\mathcal{M}_{\left[t_{1}, t_{2}\right]}$ by a countable collection $\left\{P_{i}\right\}_{i}$ of such neighborhoods, with a subordinate partition of unity $\left\{\phi_{i}\right\}_{i}$. Let $e_{i}:\left(a_{i}, b_{i}\right) \times X_{i} \rightarrow P_{i}$ be the corresponding map. As

$$
\int_{\mathcal{M}_{\left[t_{1}, t_{2}\right]}} \phi_{i} F d \operatorname{vol}_{g_{\mathcal{M}}}=\int_{t_{1}}^{t_{2}} \int_{e_{i}\left(\{t\} \times X_{i}\right)} \phi_{i} F d \operatorname{vol}_{g(t)} d t
$$


we obtain

$$
\begin{aligned}
\int_{\mathcal{M}_{\left[t_{1}, t_{2}\right]}} F d \operatorname{vol}_{g_{\mathcal{M}}} & =\sum_{i} \int_{\mathcal{M}_{\left[t_{1}, t_{2}\right]}} \phi_{i} F d \operatorname{vol}_{g_{\mathcal{M}}} \\
& =\sum_{i} \int_{t_{1}}^{t_{2}} \int_{e_{i}\left(\{t\} \times X_{i}\right)} \phi_{i} F d \operatorname{vol}_{g(t)} d t \\
& =\int_{t_{1}}^{t_{2}} \sum_{i} \int_{e_{i}\left(\{t\} \times X_{i}\right)} \phi_{i} F d \operatorname{vol}_{g(t)} d t \\
& =\int_{t_{1}}^{t_{2}} \int_{\mathcal{M}_{t}} F d \operatorname{vol}_{g(t)} d t .
\end{aligned}
$$

This proves the lemma.

We now prove some results about the behavior of volume in Ricci flow spacetimes.

Proposition 5.5. Let $\mathcal{M}$ be a Ricci flow spacetime. Suppose that

(a) the quasi-parabolic metric $g_{\mathcal{M}}^{\mathrm{qp}}$ of Definition 1.7 is complete along worldlines;

(b) if $B_{t} \subset \mathcal{M}_{t}$ is the set of points whose maximal worldline does not extend backward to time zero, then $B_{t}$ has measure zero with respect to $d \operatorname{vol}_{g(t)}$, for each $t \geqslant 0$;

(c) the initial time slice $\mathcal{M}_{0}$ has volume $\mathcal{V}(0)<\infty$;

(d) the scalar curvature is proper and bounded below on time slabs $\mathcal{M}_{\leqslant T}$, and the initial time slice has scalar curvature bounded below by $-C$, with $C \geqslant 0$.

Let $\mathcal{V}(t)$ be the volume of $\mathcal{M}_{t}$. Then, the following conditions hold:

(1) $\mathcal{V}(t) \leqslant \mathcal{V}(0)(1+2 C t / n)^{n / 2}$;

(2) $R$ is integrable on $\mathcal{M}_{\left[t_{1}, t_{2}\right]}$;

(3) for all $t_{1}<t_{2}$,

$$
\mathcal{V}\left(t_{2}\right)-\mathcal{V}\left(t_{1}\right)=-\int_{\mathcal{M}_{\left[t_{1}, t_{2}\right]}} R d \operatorname{vol}_{g_{\mathcal{M}}}
$$

(4) the volume function $\mathcal{V}(t)$ is absolutely continuous;

(5) given $0 \leqslant t_{1} \leqslant t_{2}<\infty$, we have

$$
\mathcal{V}\left(t_{2}\right)-\mathcal{V}\left(t_{1}\right) \leqslant \frac{C}{1+2 C t_{1} / n}\left(1+\frac{2 C t_{2}}{n}\right)^{n / 2} \mathcal{V}(0)\left(t_{2}-t_{1}\right)
$$

Proof. Given $0 \leqslant t_{1}<t_{2}<\infty$, there is a partition

$$
\mathcal{M}_{\left[t_{1}, t_{2}\right]}=\mathcal{M}_{\left[t_{1}, t_{2}\right]}^{\prime} \cup \mathcal{M}_{\left[t_{1}, t_{2}\right]}^{\prime \prime} \cup \mathcal{M}_{\left[t_{1}, t_{2}\right]}^{\prime \prime \prime},
$$

where

(1) a point in $\mathcal{M}_{\left[t_{1}, t_{2}\right]}^{\prime}$ has a worldline that intersects both $M_{t_{1}}$ and $M_{t_{2}}$;

(2) a point in $\mathcal{M}_{\left[t_{1}, t_{2}\right]}^{\prime \prime}$ has a worldline that intersects $M_{t_{1}}$ but not $M_{t_{2}}$;

(3) a point in $\mathcal{M}_{\left[t_{1}, t_{2}\right]}^{\prime \prime \prime}$ has a worldline that does not intersect $M_{t_{1}}$. 
By our assumptions,

$$
\mathcal{M}_{\left[t_{1}, t_{2}\right]}^{\prime \prime \prime} \subset \bigcup_{t \in\left[t_{1}, t_{2}\right]} B_{t}
$$

has measure zero with respect to $d \operatorname{vol}_{g_{\mathcal{M}}}$; cf. the proof of Lemma 5.4. Put

$$
X_{1}=\mathcal{M}_{\left[t_{1}, t_{2}\right]}^{\prime} \cap M_{t_{1}} \quad \text { and } \quad X_{2}=\mathcal{M}_{\left[t_{1}, t_{2}\right]}^{\prime \prime} \cap M_{t_{1}}
$$

For $s \in\left[t_{1}, t_{2}\right]$, there is a natural embedding $i_{s}: X_{1} \rightarrow \mathcal{M}_{s}$ coming from flowing along worldlines. The complement $M_{t_{2}} \backslash i_{t_{2}}\left(X_{1}\right)$ has measure zero. Thus,

$$
\mathcal{V}\left(t_{2}\right)=\int_{M_{t_{2}}} d \operatorname{vol}_{g\left(t_{2}\right)}=\int_{X_{1}} i_{t_{2}}^{*} d \operatorname{vol}_{g\left(t_{2}\right)}
$$

and

$$
\mathcal{V}\left(t_{1}\right)=\int_{X_{1}} d \operatorname{vol}_{g\left(t_{1}\right)}+\int_{X_{2}} d \operatorname{vol}_{g\left(t_{1}\right)} .
$$

Given $x \in X_{1}$, let $\gamma_{x}:\left[t_{1}, t_{2}\right] \rightarrow \mathcal{M}_{\left[t_{1}, t_{2}\right]}$ be its worldline. Put

$$
J_{s}(x)=\frac{i_{s}^{*} d \operatorname{vol}_{g(s)}}{d \operatorname{vol}_{g\left(t_{1}\right)}}(x) .
$$

From the Ricci flow equation,

$$
J_{s}(x)=\exp \left(-\int_{t_{1}}^{s} R\left(\gamma_{x}(u)\right) d u\right) .
$$

Using Lemma 5.2,

$$
\begin{aligned}
\mathcal{V}\left(t_{2}\right) & =\int_{X_{1}} J_{t_{2}}(x) d \operatorname{vol}_{g\left(t_{1}\right)}(x) \leqslant \int_{X_{1}} \exp \left(\int_{t_{1}}^{t_{2}} \frac{C}{1+2 C u / n} d u\right) d \operatorname{vol}_{g\left(t_{1}\right)} \\
& =\mathcal{V}\left(t_{1}\right)\left(\frac{1+2 C t_{2} / n}{1+2 C t_{1} / n}\right)^{n / 2} .
\end{aligned}
$$

When $t_{1}=0$, this proves part (1) of the proposition.

Next,

$$
\begin{aligned}
\int_{X_{1}}\left(i_{t_{2}}^{*} d \operatorname{vol}_{g\left(t_{2}\right)}-d \operatorname{vol}_{g\left(t_{1}\right)}\right) & =\int_{X_{1}}\left(J_{t_{2}}(x)-1\right) d \operatorname{vol}_{g\left(t_{1}\right)}(x) \\
& =\int_{X_{1}} \int_{t_{1}}^{t_{2}} \frac{d J_{s}(x)}{d s} d s d \operatorname{vol}_{g\left(t_{1}\right)}(x) \\
& =-\int_{X_{1}} \int_{t_{1}}^{t_{2}} R\left(\gamma_{x}(s)\right) J_{s}(x) d s d \operatorname{vol}_{g\left(t_{1}\right)}(x) \\
& =-\int_{t_{1}}^{t_{2}} \int_{X_{1}} R i_{s}^{*} d \operatorname{vol}_{g(s)} d s \\
& =-\int_{\mathcal{M}_{\left[t_{1}, t_{2}\right]}^{\prime}} R d \operatorname{vol}_{g_{\mathcal{M}}},
\end{aligned}
$$


where we applied Lemma 5.4 with $F=R 1_{\mathcal{M}_{\left[t_{1}, t_{2}\right]}^{\prime}}$ in the last step.

Given $x \in X_{2}$, let $e(x) \in\left(t_{1}, t_{2}\right)$ be the supremal extension time of its worldline. From the completeness of $g_{\mathcal{M}}^{\mathrm{qp}}$ on worldlines that do not terminate at the time-zero slice,

$$
\int_{t_{1}}^{e(x)} R\left(\gamma_{x}(u)\right) d u=\infty
$$

Thus, $\lim _{s \rightarrow e(x)} J_{s}(x)=0$, so

$$
\begin{aligned}
-\int_{X_{2}} d \operatorname{vol}_{g\left(t_{1}\right)} & =\int_{X_{2}} \int_{t_{1}}^{e(x)} \frac{d J_{s}(x)}{d s} d s d \operatorname{vol}_{g\left(t_{1}\right)}(x) \\
& =-\int_{X_{2}} \int_{t_{1}}^{e(x)} R\left(\gamma_{x}(s)\right) J_{s}(x) d s d \operatorname{vol}_{g\left(t_{1}\right)}(x) \\
& =-\int_{X_{2}} \int_{t_{1}}^{e(x)} R d s i_{s}^{*} d \operatorname{vol}_{g(s)} \\
& =-\int_{\mathcal{M}_{\left[t_{1}, t_{2}\right]}^{\prime \prime}} R d \operatorname{vol}_{\mathcal{M}_{\mathcal{M}}} .
\end{aligned}
$$

Part (3) of the proposition follows from combining equations (5.6), (5.7), (5.10) and (5.12). Part (2) is now an immediate consequence.

By Lemma 5.4 and part (3) of the proposition, the function $t \mapsto \int_{\mathcal{M}_{t}} R d$ vol is locally$L^{1}$ on $[0, \infty)$ with respect to the Lebesgue measure. This implies part (4).

To prove part (5), using Lemma 5.2 and parts (1) and (3), we have

$$
\begin{aligned}
\mathcal{V}\left(t_{2}\right)-\mathcal{V}\left(t_{1}\right) & =-\int_{t_{1}}^{t_{2}} \int_{\mathcal{M}_{t}} R d \operatorname{vol}_{g(t)} d t \leqslant \int_{t_{1}}^{t_{2}} \frac{C}{1+2 C t / n} \mathcal{V}(t) d t \\
& \leqslant \frac{C}{1+2 C t_{1} / n}\left(1+\frac{2}{n} C t_{2}\right)^{n / 2} \mathcal{V}(0)\left(t_{2}-t_{1}\right) .
\end{aligned}
$$

This proves the proposition.

\subsection{Basic structural properties of singular Ricci flows}

In this subsection we collect a number of properties of singular Ricci flows, the latter being in the sense of Definition 1.4. We first show the completeness of the quasi-parabolic metric.

Lemma 5.13. If $\mathcal{M}$ is a singular Ricci flow, then the quasi-parabolic metric $g_{\mathcal{M}}^{\mathrm{qp}}$ of Definition 1.7 is complete away from the time-zero slice. 
Proof. Suppose that $\gamma:[0, \infty) \rightarrow \mathcal{M}$ is a curve that goes to infinity in $\mathcal{M}$, with to $\gamma$ bounded away from zero. We want to show that its quasi-parabolic length is infinite. If $\mathfrak{t}_{\circ} \gamma$ is not bounded, then the quasi-parabolic length of $\gamma$ is infinite from the definition of $g_{\mathcal{M}}^{\mathrm{qp}}$, so we may assume that $\mathrm{t}_{\circ} \gamma$ takes values in some interval $[0, T]$. Since $R$ is proper and bounded below on $\mathcal{M}_{\leqslant T}$, we have $\lim _{s \rightarrow \infty} R(\gamma(s))=\infty$. After truncating the initial part of $\gamma$, we may assume that $R(\gamma(s)) \geqslant r(T)^{-2}$ for all $s$. In particular, each point $\gamma(s)$ is in a canonical neighborhood. Now,

$$
\frac{d R(\gamma(s))}{d s}=\frac{\partial R}{\partial t} \frac{d t}{d s}+\left\langle\nabla R, \gamma^{\prime}\right\rangle_{g}
$$

so

$$
\left|\frac{d R(\gamma(s))}{d s}\right|^{2} \leqslant 2\left(\left|\frac{\partial R}{\partial t}\right|^{2}\left|\frac{d t}{d s}\right|^{2}+|\nabla R|_{g}^{2}\left|\gamma^{\prime}\right|_{g}^{2}\right) .
$$

The gradient estimates in (A.8), of the form

$$
|\nabla R|<\text { const. } R^{3 / 2} \text { and }\left|\partial_{t} R\right|<\text { const. } R^{2},
$$

are valid for points in a canonical neighborhood of a singular Ricci flow solutions. Then

$$
\left|\frac{d R(\gamma(s))}{d s}\right|^{2} \leqslant C R^{2}\left|\frac{d \gamma}{d s}\right|_{g_{\mathcal{M}}^{\mathrm{qp}}}^{2}
$$

for some universal $C<\infty$. We deduce that

$$
\int_{0}^{\infty}\left|R^{-1} \frac{d R}{d s}\right| d s \leqslant C^{1 / 2} \int_{0}^{\infty}\left|\frac{d \gamma}{d s}\right|_{g_{\mathcal{M}}^{\mathrm{qp}}} d s .
$$

Since the left-hand side is infinite, the quasi-parabolic length of $\gamma$ must be infinite. This proves the lemma.

The next lemma gives the existence of unscathed forward and backward parabolic neighborhoods of a certain size around a point, along with geometric bounds on those neighborhoods.

Lemma 5.14. Let $\mathcal{M}$ be a singular Ricci flow. Given $T<\infty$, there are numbers $\sigma=$ $\sigma(T)>0, i_{0}=i_{0}(T)>0$ and $A_{k}=A_{k}(T)<\infty, k \geqslant 0$, with the following property. If $m \in \mathcal{M}$ and $\mathfrak{t}(m) \leqslant T$, put $Q=|R(m)|+r(\mathfrak{t}(m))^{-2}$. Then,

(1) the forward and backward parabolic balls $P_{+}\left(m, \sigma Q^{-1 / 2}\right)$ and $P_{-}\left(m, \sigma Q^{-1 / 2}\right)$ are unscathed;

(2) $|\mathrm{Rm}| \leqslant A_{0} Q$, inj $\geqslant i_{0} Q^{-1 / 2}$ and $\left|\nabla^{k} \mathrm{Rm}\right| \leqslant A_{k} Q^{1+k / 2}$ on the union

$$
P_{+}\left(m, \sigma Q^{-1 / 2}\right) \cup P_{-}\left(m, \sigma Q^{-1 / 2}\right)
$$

of the forward and backward parabolic balls. 
Proof. The proof is the same as that of Lemma 3.1.

The next two propositions characterize the high-scalar-curvature part of a time slice.

Proposition 5.15. Let $\mathcal{M}$ be a singular Ricci flow. For every $\varepsilon_{1}>0$, there is a scale function $r_{1}:[0, \infty) \rightarrow(0, \infty)$ with $r_{1}(t) \leqslant r(t)$ such that for every point $m \in \mathcal{M}$ with $R(m)>r_{1}(\mathfrak{t}(m))^{-2}$ the $\varepsilon_{1}$-canonical neighborhood assumption holds, and moreover $(\mathcal{M}, m)$ is $\varepsilon_{1}$-modelled on a $\varkappa$-solution. (Recall that here $\varkappa=\varkappa(t)$, i.e. we are suppressing the time dependence in our notation.)

Proof. The proof is similar to the proof of [44, Theorem 52.7]. Suppose that the proposition is not true. Then, there is a sequence of singular Ricci flows $\left\{\mathcal{M}_{j}\right\}_{j=1}^{\infty}$, along with points $m_{j} \in \mathcal{M}_{j}$, that together provide a counterexample. After passing to a subsequence, we extract a limiting flow, as in Step 2 of the proof of [44, Theorem 52.7]. In that proof, the existence of a limiting flow used a point selection argument from Step 1 of the proof. In the present case, because of the canonical neighborhood assumption in the definition of singular Ricci flow, we do not have to perform point selection in order to extract a limiting flow.

Similarly, using the existing canonical neighborhood assumption simplifies Step 2 of the proof of [44, Theorem 52.7]. The rest of the proof of the proposition is the same as in Steps 3 and 4 of the proof of [44, Theorem 52.7].

As a further point, time slices are not assumed to be compact as in [44, Theorem 52.7], and are therefore not necessarily complete. To deal with this, there are places in the proof where one applies Lemma 5.14 to compensate for any incompleteness.

Proposition 5.16. Let $\mathcal{M}$ be a singular Ricci flow. For any $T<\infty$ and $\hat{\varepsilon}>0$, there exist $C_{1}=C_{1}(\hat{\varepsilon}, T)<\infty$ and $\bar{R}=\bar{R}(\hat{\varepsilon}, T)<\infty$ such that for every $t \leqslant T$ each connected component of the time slice $\mathcal{M}_{t}$ has finitely many ends, each of which is an $\hat{\varepsilon}-h o r n$. Morever, for every $\bar{R}^{\prime} \geqslant \bar{R}$, the superlevel set $\mathcal{M}_{t}^{>\bar{R}^{\prime}}=\left\{m \in \mathcal{M}_{t}: R(m)>\bar{R}^{\prime}\right\}$ is contained in a finite disjoint union of properly embedded 3-dimensional submanifolds $\left\{N_{i}\right\}_{i=1}^{k}$ with boundary such that the following conditions are satisfied:

(1) each $N_{i}$ is contained in the superlevel set $\mathcal{M}_{t}^{>C_{1}^{-1} \bar{R}^{\prime}}$;

(2) the boundary $\partial N_{i}$ has scalar curvature in the interval $\left(C_{1}^{-1} \bar{R}^{\prime}, C_{1} \bar{R}^{\prime}\right)$;

(3) for each $i$ one of the following holds:

(3a) $N_{i}$ is diffeomorphic to $S^{1} \times S^{2}$ or $I \times S^{2}$ and consists of $\hat{\varepsilon}$-neck points; note that here the interval I can be open (a double horn), closed (a tube) or half-open ( horn);

(3b) $N_{i}$ is diffeomorphic to $D^{3}=\bar{B}^{3}$ or $\mathbb{R P}^{3} \backslash B^{3}$ and its boundary $\partial N_{i} \simeq S^{2}$ consists of $\hat{\varepsilon}$-neck points;

(3c) $N_{i}$ is diffeomorphic to $S^{3}, \mathbb{R P}^{3}$ or $\mathbb{R P}^{3} \# \mathbb{R} \mathbb{P}^{3}$; 
(3d) $N_{i}$ is diffeomorphic to a spherical space form other than $S^{3}$ or $\mathbb{R P}^{3}$, and $(\mathcal{M}, x)$ is $\hat{\varepsilon}$-modelled on a shrinking round spherical space form, for all $x \in N_{i}$;

(4) in cases (3b) and (3c), if $S_{i} \subset N_{i}$ is a subset consisting of non- $\hat{\varepsilon}-n e c k$ points such that for any two distinct elements $s_{1}, s_{2} \in S_{i}$ we have $d_{\mathcal{M}_{t}}\left(s_{1}, s_{2}\right)>C_{1} R^{-1 / 2}\left(s_{1}\right)$, then the cardinality $\left|S_{i}\right|$ is at most 1 in case $(3 \mathrm{~b})$ and at most 2 in case $(3 \mathrm{c})$;

(5) each $N_{i}$ with non-empty boundary has volume at least $C_{1}^{-1}\left(\bar{R}^{\prime}\right)^{-3 / 2}$.

Proof. The proof is the same as in $[44, \S 67]$.

We now prove a statement about preservation of connected components when going backwards in time.

Proposition 5.17. Let $\mathcal{M}$ be a singular Ricci flow. If $\gamma_{0}, \gamma_{1}:\left[t_{0}, t_{1}\right] \rightarrow \mathcal{M}$ are timepreserving curves, and $\gamma_{0}\left(t_{1}\right)$ and $\gamma_{1}\left(t_{1}\right)$ lie in the same connected component of $\mathcal{M}_{t_{1}}$, then $\gamma_{0}(t)$ and $\gamma_{1}(t)$ lie in the same connected component of $\mathcal{M}_{t}$ for every $t \in\left[t_{0}, t_{1}\right]$.

Proof. The idea of the proof is to consider the values of $t$ for which $\gamma_{0}(t)$ can be joined to $\gamma_{1}(t)$ in $\mathcal{M}_{t}$, and the possible curves $c_{t}$ in $\mathcal{M}_{t}$ that join them. Among all such curves $c_{t}$, we look at one which minimizes the maximum value of scalar curvature along the curve. Call this threshold value of scalar curvature $R_{\text {crit }}(t)$. We will argue that $c_{t}$ can only intersect the high-scalar-curvature part of $\mathcal{M}_{t}$ in its neck-like regions. But the scalar curvature in a neck-like region is strictly decreasing when one goes backward in time; this will imply that $R_{\text {crit }}(t)$, when large, is decreasing when one goes backward in time, from which the lemma will follow.

To begin the formal proof, suppose that the lemma is false. Let $S \subset\left[t_{0}, t_{1}\right]$ be the set of times $t \in\left[t_{0}, t_{1}\right]$ such that $\gamma_{0}(t)$ and $\gamma_{1}(t)$ lie in the same connected component of $\mathcal{M}_{t}$; note that $S$ is open. Put $\hat{t}=\inf \left\{t:\left[t, t_{1}\right] \subset S\right\}$. Then $\hat{t}>\frac{1}{100}$ since $\mathcal{M}_{[0,1 / 100]}$ is a product. Also, for $i \in\{0,1\}$ and $t \in\left[\hat{t}, t_{1}\right]$ close to $\hat{t}$, if $\widehat{\gamma}_{i}(t) \in \mathcal{M}_{t}$ is the worldline of $\gamma_{i}(\hat{t})$ at time $t$, then $\widehat{\gamma}_{i}(t)$ lies in the same connected component of $\mathcal{M}_{t}$ as $\gamma_{i}(t)$. Therefore, after reducing $t_{1}$ if necessary, we may assume without loss of generality that $\gamma_{i}$ is a worldline.

For $t \in\left[t_{0}, t_{1}\right]$ and $\bar{R}<\infty$, put $\mathcal{M}_{t}^{\leqslant \bar{R}}=\left\{m \in \mathcal{M}_{t}: R(m) \leqslant \bar{R}\right\}$. For $t \in\left(\hat{t}, t_{1}\right]$, let $\mathcal{R}_{t}$ be the set of $\bar{R} \in \mathbb{R}$ such that $\gamma_{0}(t)$ and $\gamma_{1}(t)$ lie in the same connected component of $\mathcal{M}_{t}^{\leqslant \bar{R}}$. Put $R_{\text {crit }}(t)=\inf \mathcal{R}_{t}$. Since $R: \mathcal{M}_{t} \rightarrow \mathbb{R}$ is proper, the sets $\left\{\mathcal{M}_{t}^{\leqslant \bar{R}}\right\}_{\bar{R}>R_{\text {crit }}(t)}$ are compact and nested, which implies that $\gamma_{0}(t)$ and $\gamma_{1}(t)$ lie in the same component of

$$
\mathcal{M}_{t}^{\leqslant R_{\text {crit }}(t)}=\bigcap_{\bar{R}>R_{\text {crit }}(t)} \mathcal{M}_{t}^{\leqslant \bar{R}}
$$

i.e. $R_{\text {crit }}(t) \in \mathcal{R}_{t}$.

By Lemma 5.14, there is a $C<\infty$ such that, for any $t \in\left(\frac{1}{100}, t_{1}\right]$ and any $m \in \mathcal{M}_{t}$, there is a $\tau=\tau\left(t_{1}, R(m)\right)>0$, where $\tau$ is a continuous function which is non-increasing in 
$R(m)$, such that the worldline $\gamma_{m}$ of $m$ is defined and satisfies

$$
\left|\frac{d R\left(\gamma_{m}(t)\right)}{d t}\right|<C \cdot R(m)^{2}
$$

in the time interval $(t-\tau, t+\tau)$. Now for $t \in\left(\hat{t}, t_{1}\right]$ and $t^{\prime}$ satisfying $\left|t^{\prime}-t\right|<\tau\left(t_{1}, R_{\text {crit }}(t)\right)$, let $Z_{t, t^{\prime}} \subset \mathcal{M}_{t^{\prime}}$ denote the result of flowing $\mathcal{M}_{t}^{\leqslant R_{\text {crit }}(t)}$ under $\partial_{\mathfrak{t}}$ for an elapsed time $t^{\prime}-t$. Then $Z_{t, t^{\prime}}$ is well defined and contains $\gamma_{0}\left(t^{\prime}\right)$ and $\gamma_{1}\left(t^{\prime}\right)$ in the same component, so

$$
R_{\text {crit }}\left(t^{\prime}\right) \leqslant \max _{Z_{t, t^{\prime}}} R \leqslant R_{\text {crit }}(t)+C \cdot R_{\text {crit }}(t)^{2} \cdot\left|t-t^{\prime}\right| .
$$

This implies that $R_{\text {crit }}:\left(\hat{t}, t_{1}\right] \rightarrow \mathbb{R}$ is locally Lipschitz (in particular continuous) and that

$$
R_{\text {crit }}(t) \longrightarrow \infty
$$

as $t \rightarrow \hat{t}$ from the right; otherwise, there would be a sequence $t_{i} \rightarrow \hat{t}$ along which $R_{\text {crit }}$ is uniformly bounded above by some $\widehat{R}<\infty$, which would allow us to construct $Z_{t_{i}, \hat{t}}$ whenever $\left|\hat{t}-t_{i}\right|<\tau\left(t_{1}, \widehat{R}\right)$, contradicting the definition of $\hat{t}$.

We now concentrate on $t$ close to $\hat{t}$. Suppose that, for some $t \in\left(\hat{t}, t_{1}\right]$, we have

$$
R_{\text {crit }}(t) \gg \max \left(r^{-2}\left(t_{1}\right), \max _{\left[t_{0}, t_{1}\right]} R \circ \gamma_{0}, \max _{\left[t_{0}, t_{1}\right]} R \circ \gamma_{1}\right)
$$

Then, by Proposition 5.16, the superlevel set $\mathcal{M}_{t}^{>\left(R_{\text {crit }}(t)-1\right)}$ is contained in a finite union of components $\left\{N_{i, t}\right\}_{i=1}^{k_{t}}$, each diffeomorphic to one of the possibilities (3a)-(3d) in the statement of the proposition. Let $X_{t}$ be the result of removing from $\mathcal{M}_{t}$ the interior of each $N_{i, t}$ that is not of type (3a). Since $\gamma_{0}(t)$ and $\gamma_{1}(t)$ lie outside $\bigcup_{i=1}^{k_{t}} N_{i, t}$, and each $N_{i}$ of type $(3 \mathrm{~b})-(3 \mathrm{~d})$ has at most one boundary component, it follows that $\gamma_{0}(t)$ and $\gamma_{1}(t)$ lie in the same connected component of $X_{t}$.

For $t^{\prime}<t$ close to $t$, let $X_{t^{\prime}} \subset \mathcal{M}_{t^{\prime}}$ be the result of flowing $X_{t}$ under $\partial_{t}$. Now,

$$
Y_{t}=X_{t} \cap \mathcal{M}_{t}^{>\left(R_{\mathrm{crit}}(t)-1\right)}
$$

consists of $\varepsilon$-neck points, and at such a point the scalar curvature is strictly increasing as a function of time. Hence, there is a $\tau_{1}>0$ such that the worldline $\gamma_{m}:\left[t-\tau_{1}, t\right] \rightarrow \mathcal{M}$ of any $m \in Y_{t}$ satisfies

$$
R\left(\gamma_{m}\left(t^{\prime}\right)\right)<R(m) \leqslant R_{\text {crit }}(t)
$$

for $t^{\prime} \in\left[t-\tau_{1}, t\right)$. This implies that $R_{\text {crit }}\left(t^{\prime}\right)<R_{\text {crit }}(t)$ when $t^{\prime}<t$ is close to $t$, again under the assumption (5.20), which contradicts (5.19). 
We now state a result about connecting a point in a singular Ricci flow $\mathcal{M}$ to the time-zero slice by a curve whose length, with respect to the spacetime metric $g_{\mathcal{M}}$, is quantitatively bounded, and along which the scalar curvature is quantitatively bounded. This is similar to Proposition 3.3.

Proposition 5.21. Let $\mathcal{M}$ be a singular Ricci flow. Given $T, R_{0}<\infty$, there are constants $L=L\left(R_{0}, T\right)<\infty$ and $R_{1}=R_{1}\left(R_{0}, T\right)<\infty$ with the following property. Suppose that $R\left(m_{0}\right) \leqslant R_{0}$, with $t_{0}=\mathfrak{t}\left(m_{0}\right) \leqslant T$. Then, there is a time-preserving curve $\gamma:\left[0, t_{0}\right] \rightarrow \mathcal{M}$ with $\gamma\left(t_{0}\right)=m_{0}$ and length $(\gamma) \leqslant L$ such that $R(\gamma(t)) \leqslant R_{1}$ for all $t \in\left[0, t_{0}\right]$.

Proof. The proof is the same as that of Proposition 3.3.

Finally, we give a compactness result for the space of singular Ricci flows.

Proposition 5.22. Let $\left\{\mathcal{M}^{i}\right\}_{i=1}^{\infty}$ be a sequence of singular Ricci flows with a fixed choice of parameters $\varepsilon, r$ and $\varkappa$ whose initial conditions $\left\{\mathcal{M}_{0}^{i}\right\}_{i=1}^{\infty}$ lie in a compact family in the smooth topology. Then, a subsequence of $\left\{\mathcal{M}^{i}\right\}_{i=1}^{\infty}$ converges, in the sense of Theorem 4.1, to a singular Ricci flow $\mathcal{M}^{\infty}$.

Proof. Using Proposition 5.21, the proof is the same as that of Proposition 4.1.

Remark 5.23. In the setting of Proposition 5.22, if we instead assume that the (normalized) initial conditions have a uniform upper volume bound, then we can again take a convergent subsequence to get a limit Ricci flow spacetime $\mathcal{M}^{\infty}$. In this case the time-zero slice $\mathcal{M}_{0}^{\infty}$ will generally only be $C^{1, \alpha}$-regular, but $\mathcal{M}^{\infty}$ will be smooth on $\mathfrak{t}^{-1}(0, \infty)$.

\section{Stability of necks}

In this section we fix $\varkappa>0$; the dependence of various constants on this choice of $\varkappa$ is implicit. We recall the notion of a $\varkappa$-solution from $\S$ A.5. In this section we establish a new dynamical stability property of caps and necks in $\varkappa$-solutions, which we will use in $\S 7$ to show that a bad worldline $\gamma: I \rightarrow \mathcal{M}$ is confined to a cap region as $t$ approaches the blow-up time inf $I$.

Conceptually speaking, the stability assertion is that among pointed $\varkappa$-solutions, the round cylinder is an attractor under backward flow; similarly, under forward flow, non-neck points form an attractor. The rough idea of the argument is as follows. Suppose that $\delta \ll 1$ and $\left(x_{0}, 0\right)$ is a $\delta$-neck in a $\varkappa$-solution $\mathcal{M}$. Then, $\left(x_{0}, t\right)$ will also be neck-like, as long as $t<0$ is not too negative. One also knows that $\mathcal{M}$ has an asymptotic soliton as $t \rightarrow-\infty$, which is a shrinking round cylinder. Thus, $\mathcal{M}$ tends toward neck-like geometry as $t$ approaches $-\infty$, which is the desired stability property. However, there is a catch 
here: the asymptotic soliton is a pointed limit of a sequence where the basepoints are not fixed, and so a priori it says nothing about the asymptotic geometry near $\left(x_{0}, t\right)$ as $t \rightarrow-\infty$. To address this, we exploit the behavior of the $l$-function.

\subsection{The main stability asssertion}

We recall the notation $\widehat{\mathcal{M}}(t)$ from $\S A .1$ for the parabolic rescaling of a Ricci flow spacetime $\mathcal{M}$. We recall the notation $\mathrm{Cyl}$ and Sphere from $\S$ A.1 for the standard Ricci flow solutions. We also recall the notion of one Ricci flow spacetime being $\varepsilon$-modelled on another one, from $\S$ A.2.

TheOREM 6.1. There is a $\delta_{\text {neck }}=\delta_{\text {neck }}(\varkappa)>0$, and for all $\delta_{0}, \delta_{1} \leqslant \delta_{\text {neck }}$ there is a $T=T\left(\delta_{0}, \delta_{1}, \varkappa\right) \in(-\infty, 0)$ with the following property. Suppose that

(a) $\mathcal{M}$ is a $\varkappa$-solution with non-compact time slices;

(b) $\left(x_{0}, 0\right) \in \mathcal{M}$;

(c) $R\left(x_{0}, 0\right)=1$;

(d) $\left(\mathcal{M},\left(x_{0}, 0\right)\right)$ is a $\delta_{0}$-neck.

Then, either $\mathcal{M}$ is isometric to the $\mathbb{Z}_{2}$-quotient of a shrinking round cylinder, or for all $t \in(-\infty, T]$,

(1) $\left(\mathcal{M},\left(x_{0}, t\right)\right)$ is a $\delta_{1}$-neck and

(2) $\left(\widehat{\mathcal{M}}(-t),\left(x_{0},-1\right)\right)$ is $\delta_{1}$-close to $\left(\mathrm{Cyl},\left(y_{0},-1\right)\right)$, where $y_{0} \in S^{2} \times \mathbb{R}$.

We recall the notion of a generalized neck from $\S$ A.2.

COROLlary 6.2. If $\delta_{\text {neck }}=\delta_{\text {neck }}(\varkappa)$ as in the previous theorem, then there is a $T=$ $T(\varkappa)<(-\infty, 0)$ such that, if $\mathcal{M}$ is a $\varkappa$-solution with non-compact time slices, $\left(x_{0}, 0\right) \in \mathcal{M}$, $R\left(x_{0}, 0\right)=1$ and $\left(x_{0}, 0\right)$ is a generalized $\delta_{\text {neck }}-n e c k$, then $\left(x_{0}, T\right)$ is a generalized $\frac{1}{4} \delta_{\text {neck }}$ neck, and $R\left(x_{0}, T\right)<\frac{1}{4}$.

\subsection{Convergence of $l$-functions, asymptotic $l$-functions and asymptotic solitons}

In this subsection we prove some preparatory results about the convergence of $l$-functions, with regard to a convergent sequence of 3 -dimensional $\varkappa$-solutions.

Suppose that we are given that $R\left(x_{1}, t_{1}\right) \leqslant C$ at some point $\left(x_{1}, t_{1}\right)$ in a $\varkappa$-solution. By compactness of the space of normalized pointed $\varkappa$-solutions (see $\S$ A.5), we obtain $R\left(x, t_{1}\right) \leqslant F\left(C, d_{t_{1}}\left(x, x_{1}\right)\right)$ for some universal function $F$. Since $R$ is pointwise nondecreasing in time in a $\varkappa$-solution, we also have $R(x, t) \leqslant F\left(C, d_{t_{1}}\left(x, x_{1}\right)\right.$ whenever $t \leqslant t_{1}$. 
Lemma 6.3. (Curvature bound at basepoint) Let $\left\{\left(\mathcal{M}^{j},\left(x_{j}, 0\right)\right)\right\}_{j}$ be a sequence of pointed $\varkappa$-solutions, with $\sup _{j} R\left(x_{j}, 0\right)<\infty$, and let $l_{j}: \mathcal{M}_{<0}^{j} \rightarrow(0, \infty)$ be the reduced distance function with spacetime basepoint at $\left(x_{j}, 0\right)$. Then, after passing to a subsequence, we have convergence of tuples

$$
\left(\mathcal{M}^{j},\left(x_{j}, 0\right), l_{j}\right) \rightarrow\left(\mathcal{M}^{\infty},\left(x_{\infty}, 0\right), l_{\infty}\right)
$$

Here the Ricci flow convergence is the usual smooth convergence on parabolic balls, and the $l_{j}$ 's converge to a locally Lipschitz function $l_{\infty}$ uniformly on compact subsets of $\mathcal{M}_{<0}^{\infty}$, after composition with the comparison map.

Proof. Since $R\left(x_{j}, 0\right)$ is uniformly bounded above, the compactness of the space of normalized pointed $\varkappa$-solutions implies that a subsequence, which we relabel as

$$
\left\{\left(\mathcal{M}^{j},\left(x_{j}, 0\right)\right)\right\}_{j},
$$

converges in the pointed smooth topology. Along the curve $\left\{x_{j}\right\} \times(-\infty, 0) \subset \mathcal{M}^{j}$, the scalar curvature is bounded above by $R\left(x_{j}, 0\right)$. For any $a<b<0$, this gives a uniform upper bound on $l_{j}$ on $\left\{x_{j}\right\} \times[a, b] \subset \mathcal{M}^{j}$. From (A.5), we get that $l$ is uniformly bounded on balls of the form $B\left(x_{j}, b, r\right) \subset \mathcal{M}_{b}^{j}$. Then, from (A.6), we get that $l$ is uniformly bounded on parabolic balls of the form $P\left(x_{j}, b, r, a-b\right) \subset \mathcal{M}^{j}$. Using (A.4) and passing to a subsequence, we get convergence of $\left\{l_{j}\right\}_{j}$ to some $l_{\infty}$, uniformly on compact subsets of $\mathcal{M}_{<0}^{\infty}$.

Proposition 6.4. (No curvature bound at basepoint) Let $\left\{\left(\mathcal{M}^{j},\left(x_{j}, 0\right)\right)\right\}_{j}$ be a sequence of pointed $\varkappa$-solutions, and let $l_{j}: \mathcal{M}_{<0}^{j} \rightarrow(0, \infty)$ be the reduced distance function with spacetime basepoint at $\left(x_{j}, 0\right)$. Suppose that $\left\{\left(y_{j},-1\right)\right\}_{j}$, with $\left(y_{j},-1\right) \in \mathcal{M}_{-1}^{j}$, is a sequence satisfying $\sup _{j} l_{j}\left(y_{j},-1\right)<\infty$. Then, after passing to a subsequence, the tuples $\left(\mathcal{M}^{j},\left(y_{j},-1\right), l_{j}\right)$ converge to a tuple $\left(\mathcal{M}^{\infty},\left(y_{\infty},-1\right), l_{\infty}\right)$, where the following hold:

(1) the convergence $\left(\mathcal{M}^{j},\left(y_{j},-1\right)\right) \rightarrow\left(\mathcal{M}^{\infty},\left(y_{\infty},-1\right)\right)$ is smooth on compact subsets of $\mathcal{M}_{<0}^{\infty}$, and $\mathcal{M}^{\infty}$ is either a $\varkappa$-solution defined on $(-\infty, 0)$ or the static solution on $\mathbb{R}^{3}$;

(2) $\left\{l_{j}\right\}_{j}$ converges to a locally Lipschitz function $l_{\infty}$ uniformly on compact subsets of $\mathcal{M}_{<0}^{\infty}$, after composition with the comparison diffeomorphisms;

(3) the reduced volume functions $\widetilde{V}_{j}:(-\infty, 0) \rightarrow(0, \infty)$ converge uniformly on compact sets to the function $\widetilde{V}_{\infty}:(-\infty, 0) \rightarrow(0, \infty)$, where

$$
\widetilde{V}_{\infty}(t)=(-t)^{-n / 2} \int_{\mathcal{M}_{t}^{\infty}} e^{-l_{\infty}} d \operatorname{vol}_{g(t)} ;
$$

(4) the function $l_{\infty}$ satisfies the differential inequalities (24.6) and (24.8) in [44]. If $\widetilde{V}_{\infty}$ is constant on some time interval $\left[t_{0}, t_{1}\right] \subset(-\infty, 0)$, then $\mathcal{M}^{\infty}$ is a gradient shrinking soliton with potential $l_{\infty}$. 
Proof. From (A.6), given $a<b<0$, there is a uniform bound for $l_{j}$ on $\left\{y_{j}\right\} \times[a, b]$. From (A.3), there are bounds for $R_{j}$ on $\left\{y_{j}\right\} \times[a, b]$. Then, we get a uniform scalar curvature bound on the balls $B\left(y_{j}, b, r\right) \subset \mathcal{M}_{b}^{j}$, and hence on the parabolic neighborhoods $P\left(y_{j}, b, r,-\Delta t\right) \subset \mathcal{M}^{j}$. Taking $a \rightarrow-\infty$ and $b \rightarrow 0$, and applying a diagonal argument, we may assume that $\left\{\left(\mathcal{M}^{j},\left(y_{j},-1\right)\right)\right\}_{j}$ converges to a $\varkappa$-solution $\left(\mathcal{M}^{\infty},\left(y_{\infty},-1\right)\right)$. As in the proof of Lemma 6.3, after passing to a subsequence, we get $l_{j} \rightarrow l_{\infty}$ for some $l_{\infty}$ defined on $\mathcal{M}_{<0}^{\infty}$. The rest is as in [81] and [82].

LEMma 6.6. Let $(\mathcal{M},(x, 0), l)$ be a shrinking round cylinder with $R \equiv 1$ at $t=0$, where the $l$-function has spacetime basepoint $(x, 0)$. Then, the following statements hold:

(1) for every $t \in(-\infty, 0)$ the $l$-function $l: \mathcal{M}_{t} \rightarrow \mathbb{R}$ attains its minimum uniquely at $(x, t)$

(2) $\lim _{t \rightarrow-\infty}(\widehat{\mathcal{M}}(-t),(x,-1), l)=\left(\mathrm{Cyl},\left(x_{\infty},-1\right), l_{\infty}\right)$, where the coordinate $z$ for the $\mathbb{R}$-factor in $\mathrm{Cyl}$ satisfies $z\left(x_{\infty}\right)=0$, and

$$
l_{\infty}=1+\frac{z^{2}}{-4 t}
$$

(3) $\lim _{t \rightarrow-\infty} l(x, t)=1$.

Proof. Part (1) follows from the formula for $\mathcal{L}$-length:

$$
\mathcal{L}(\gamma)=\int_{\bar{t}}^{0} \sqrt{-t}\left(R+\left|\gamma^{\prime}(t)\right|^{2}\right) d t \geqslant \int_{\bar{t}}^{0} \sqrt{-t} R d t
$$

with equality if and only if $\gamma(t)=(x, t)$ for all $t \in[\bar{t}, 0]$. Part (2) follows from applying Lemma A.2 in $\S$ A.6 to parabolic rescalings of $\mathcal{M}$. Part (3) is now immediate.

From (6.7), we get that $l_{\infty}$ is strictly decreasing along backward worldlines, except at its minimum value 1 . In particular, if $l_{\infty}\left(x, t_{0}\right) \leqslant 1+\hat{\varepsilon}$, then $l_{\infty}\left(x, t_{1}\right)<1+\hat{\varepsilon}$ for all $t_{1}<t_{0}$. By compactness, this stability property is inherited by any tuple which approximates the shrinking round cylinder.

Lemma 6.8. For all $\hat{\varepsilon}>0$ and $A \in(0,1)$, there is a $\bar{\mu}>0$ with the following property. Suppose that $(\overline{\mathcal{M}},(x,-1), l)$ is a $\varkappa$-solution, with $l$-function based at $(x, 0)$, satisfying the following properties:

(1) $(\overline{\mathcal{M}},(x,-1), l)$ is $\bar{\mu}$-close to $\left(\mathrm{Cyl},(y,-1), l_{\infty}\right)$ on the time interval $\left[-A^{-1},-A\right]$, for some $(y,-1) \in \mathrm{Cyl}$;

(2) $l(x, t) \leqslant 1+\hat{\varepsilon}$ for all $t \in[-1,-A]$.

(Here, $l_{\infty}(y,-1)$ need not be 1.) Then, $l(x, t)<1+\hat{\varepsilon}$ for all $t \in\left[-A^{-1},-1\right]$. 
Proof. If the lemma fails then, for some $\hat{\varepsilon}>0$ and $A \in(0,1)$, there is a sequence $\left\{\left(\overline{\mathcal{M}}^{j},\left(x_{j},-1\right), l_{j}\right)\right\}_{j}$ such that, for all $j$, the following statements hold:

- $\left(\overline{\mathcal{M}}^{j},\left(x_{j},-1\right), l_{j}\right)$ is $j^{-1}$-close to $\left(\mathrm{Cyl},\left(y_{j},-1\right), l_{\infty}\right)$ on the time interval $\left[-A^{-1},-A\right]$ for some $\left(y_{j},-1\right) \in \mathrm{Cyl}_{-1}$;

- $l_{j}\left(x_{j}, t\right) \leqslant 1+\hat{\varepsilon}$ for all $t \in[-1,-A]$;

- $l_{j}\left(x_{j}, t_{j}\right) \geqslant 1+\hat{\varepsilon}$ for some $t_{j} \in\left[-A^{-1},-1\right]$.

Using (6.7) and passing to a limit, we obtain $\left(y_{\infty},-1\right) \in \mathrm{Cyl}$ with the property that $l_{\infty}\left(y_{\infty},-A\right) \leqslant 1+\hat{\varepsilon}$ and $l_{\infty}\left(y_{\infty}, t\right) \geqslant 1+\hat{\varepsilon}$ for some $t \in\left[-A^{-1},-1\right]$. But this contradicts the formula $l_{\infty}(x, t)=1+z^{2} /(-4 t)$.

\subsection{Stability of cylinders with moving basepoint}

In this subsection we prove a backward stability result for cylindrical regions, initially without fixing the basepoint. We then prove a version fixing the basepoint, which will imply Theorem 6.1.

We start with a preliminary lemma about reduced volume.

Lemma 6.9. There are $\mu>0$ and $\tau>0$ such that, if $\mathcal{M}$ is a $\varkappa$-solution defined on $(-\infty, 0]$ with $R(x, 0)=1$, then $\tilde{V}(\tau) \leqslant(4 \pi)^{3 / 2}-\mu$.

Proof. Using the monotonicity of the reduced volume, if the lemma fails then there is a sequence $\left\{\mathcal{M}^{j}\right\}_{j=1}^{\infty}$ of $\varkappa$-solutions and points $\left(x_{j}, 0\right) \in \mathcal{M}_{0}^{j}$ such that $R\left(x_{j}, 0\right)=1$ and $\widetilde{V}_{\mathcal{M}^{j}}(j) \geqslant(4 \pi)^{3 / 2}-1 / j$. After passing to a subsequence, there is pointed convergence to a pointed $\varkappa$-solution $\left(\mathcal{M}^{\infty},\left(x_{\infty}, 0\right)\right)$. Then, $R\left(x_{\infty}, 0\right)=1$ and $\widetilde{V}_{\infty}(\tau) \geqslant(4 \pi)^{3 / 2}$ for all $\tau>0$. This is a contradiction [44, proof of Proposition 39.1].

Proposition 6.10. For all $\hat{\varepsilon}>0$ and $C<\infty$, there is a $T=T(\hat{\varepsilon}, C) \in(-\infty, 0)$ with the following property. Suppose that $\mathcal{M}$ is a non-compact $\varkappa$-solution defined on $(-\infty, 0]$ and that the following conditions are satisfied:

- $(x, 0) \in \mathcal{M}_{0}$ is a point with $R(x, 0)=1$;

- $l: \mathcal{M}_{<0} \rightarrow \mathbb{R}$ is the $l$-function with spacetime basepoint $(x, 0)$;

- $t<T$, and $(y, t) \in \mathcal{M}_{t}$ is a point where the reduced distance satisfies $l(y, t) \leqslant C$.

Then, one of the following holds:

(1) the tuple $(\widehat{\mathcal{M}}(-t),(y,-1), l)$ is $\hat{\varepsilon}$-close to a triple $\left(\mathrm{Cyl},\left(y_{\infty},-1\right), l_{\infty}\right)$, where $y_{\infty} \in S^{2} \times \mathbb{R}$ and $l_{\infty}$ is the asymptotic l-function of (6.7); note that $y_{\infty}$ need not be at the minimum of $l_{\infty}$ in $\mathrm{Cyl}_{-1}$;

(2) $\mathcal{M}$ is isometric to a $\mathbb{Z}_{2}$-quotient of a shrinking round cylinder.

Proof. If the proposition were false, then for some $\hat{\varepsilon}>0$ there would be a sequence 
$\left\{\left(\mathcal{M}^{j},\left(x_{j}, 0\right)\right)\right\}_{j}$ of pointed $\varkappa$-solutions, and a sequence $\left\{T_{j}\right\}_{j}$, with $T_{j} \rightarrow-\infty$, such that

- $R\left(x_{j}, 0\right)=1$;

- there is a point $\left(y_{j}, T_{j}\right) \in \mathcal{M}_{T_{j}}^{j}$, with $l\left(y_{j}, T_{j}\right) \leqslant C$, such that both (1) and (2) fail for $t=T_{j}$.

From Lemma 6.9, there are constants $\mu>0$ and $\tau>0$ such that, for each $j$, we have $\widetilde{V}_{\mathcal{M}^{j}}(\tau) \leqslant(4 \pi)^{3 / 2}-\mu$. Using the monotonicity of the reduced volume and the existence of the asymptotic soliton, there is a uniform positive lower bound on $\widetilde{V}_{\mathcal{M}^{j}}\left(-T_{j}\right)$. After passing to a subsequence, we can find $t_{j} \in\left(T_{j}, 0\right)$ such that $T_{j} / t_{j} \rightarrow \infty$, and the reduced volume is constant to within a factor $\left(1+j^{-1}\right)$ on a time interval $\left[A_{j} t_{j}, A_{j}^{-1} t_{j}\right]$, where $A_{j} \rightarrow \infty$.

By $\S$ A.5, for each $j$ there is a point $\left(z_{j}, t_{j}\right) \in \mathcal{M}_{t_{j}}^{j}$ with $l\left(z_{j}, t_{j}\right) \leqslant \frac{3}{2}$. After passing to a subsequence, by Proposition 6.4, the sequence of parabolically rescaled flows $\left\{\left(\widehat{\mathcal{M}}^{j}\left(-t_{j}\right),\left(z_{j},-1\right)\right)\right\}_{j}$ pointed-converges to a gradient shrinking soliton $\left(\widehat{\mathcal{S}}^{\infty},\left(y_{\infty},-1\right)\right)$ that is either a $\varkappa$-solution or is flat $\mathbb{R}^{3}$. Since the reduced volume of $\left(\widehat{\mathcal{S}}^{\infty},\left(y_{\infty},-1\right)\right)$ is also bounded above by $(4 \pi)^{3 / 2}-\mu$, it cannot be flat $\mathbb{R}^{3}$. In addition, $\widehat{\mathcal{S}}^{\infty}$ cannot be a round spherical space form, as each $\mathcal{M}_{0}^{j}$ is non-compact. Also, $\widehat{\mathcal{S}}^{\infty}$ cannot be a $\mathbb{Z}_{2-}$ quotient of a shrinking round cylinder, because then $\mathcal{M}_{t}^{j}$ would contain a one-sided $\mathbb{R} \mathbb{P}^{2}$; by the classification of non-compact $\varkappa$-solutions, this would imply that $\mathcal{M}_{t}^{j}$ is isometric to the $\mathbb{Z}_{2}$-quotient of a round cylinder for all $t$, contradicting the failure of (2). Therefore $\widehat{\mathcal{S}}^{\infty}$ must be a shrinking round cylinder.

On the other hand, for each $j$, there is an asymptotic soliton of $\mathcal{M}^{j}$. By the previous argument involving spherical space forms and one-sided $\mathbb{R P}^{2}$ 's, the asymptotic soliton must be a shrinking round cylinder. It follows that, for large $j$, the reduced volume $\widetilde{V}_{\mathcal{M}^{j}}(-t)$ is nearly constant in the interval $\left(-\infty, t_{j}\right]$. Proposition 6.4 now implies that, after passing to a subsequence, $\left\{\left(\widehat{\mathcal{M}}^{j}\left(-T_{j}\right),\left(y_{j},-1\right), l_{j}\right)\right\}_{j}$ converges to a gradient shrinking soliton $\left(\hat{\mathcal{M}}^{\infty},\left(y_{\infty},-1\right), l_{\infty}\right)$, which is a $\varkappa$-solution and whose asymptotic reduced volume is that of the shrinking round cylinder. From Lemma A.2, this implies that $\widehat{\mathcal{M}}^{\infty}$ is a shrinking round cylinder, contradicting the failure of (1).

We now use the stability result of Proposition 6.10, together with Lemma 6.8, to show that worldlines that start close to necks have a nearly minimal value of $l$, provided one goes at least a certain controlled amount backward in time.

Lemma 6.11. (Basepoint stability) For all $\hat{\varepsilon}>0$, there exist $\delta=\delta(\hat{\varepsilon})>0$ and $T=$ $T(\hat{\varepsilon}) \in(-\infty, 0)$ such that, if $(\mathcal{M},(x, 0))$ is a pointed $\varkappa$-solution with $R(x, 0)=1$, and $(x, 0)$ is a $\delta$-neck, then $l(x, t)<1+\hat{\varepsilon}$ for all $t<T$.

Proof. Suppose the lemma were false. Then, for some $\hat{\varepsilon}>0$, there would be a se- 
quence $\left\{\left(\mathcal{M}^{j},\left(x_{j}, 0\right)\right)\right\}_{j}$ of pointed $\varkappa$-solutions such that

(1) $R\left(x_{j}, 0\right)=1$ and

(2) $\left(x_{j}, 0\right)$ is a $(1 / j)$-neck, but

(3) $l_{j}\left(x_{j}, \bar{t}_{j}\right) \geqslant 1+\hat{\varepsilon}$ for some $\bar{t}_{j} \leqslant-j$.

We may assume that $\hat{\varepsilon}<1$. Let $\mu_{1}>0$ be a parameter to be determined later.

By Proposition 6.10, there is a $T_{1} \in(-\infty, 0)$ such that, for large $j$ and every $(y, t) \in$ $\mathcal{M}_{\leqslant T_{1}}^{j}$ with $l_{j}(y, t)<2$, the tuple $\left(\widehat{\mathcal{M}}^{j}(-t),(y,-1), l_{j}\right)$ is $\mu_{1}$-close to $\left(\mathrm{Cyl},\left(y^{\prime},-1\right), l_{\infty}\right)$ for some $\left(y^{\prime},-1\right) \in \mathrm{Cyl}_{-1}$.

Since $\left(\mathcal{M}^{j},\left(x_{j}, 0\right)\right)$ converges to the pointed round cylinder by assumption, we have that Lemma 6.6(3) implies that there is a $T_{2} \in\left(-\infty, T_{1}\right]$ such that, for large $j$, we have $l_{j}\left(x_{j}, T_{2}\right)<1+\frac{1}{4} \hat{\varepsilon}$.

Put $t_{j}=\max \left\{t \in\left(-\infty, T_{2}\right]: l_{j}\left(x_{j}, t\right) \geqslant 1+\hat{\varepsilon}\right\}$, so $t_{j} \neq T_{2}$. Note that there is an $A \in(0,1)$, independent of $\mu_{1}$, such that $\limsup _{j \rightarrow \infty} T_{2} / t_{j}<A$, since

$$
l_{j}\left(x_{j}, T_{2}\right)<1+\frac{1}{4} \hat{\varepsilon}<1+\hat{\varepsilon}=l_{j}\left(x_{j}, t_{j}\right),
$$

in view of the time-derivative bound on $l$ given by (A.4).

For large $j$, in $\widehat{\mathcal{M}}^{j}\left(-t_{j}\right)$ we have $l_{j}\left(x_{j}, t\right) \leqslant 1+\hat{\varepsilon}$ for $t \in[-1,-A]$, but $l_{j}\left(x_{j},-1\right)=1+\hat{\varepsilon}$. Since $1+\hat{\varepsilon}<2$, we know from Proposition 6.10 that $\left(\widehat{\mathcal{M}}^{j}\left(-t_{j}\right),\left(x_{j},-1\right), l_{j}\right)$ is $\mu_{1}$-close to $\left(\mathrm{Cyl},\left(y^{\prime},-1\right), l_{\infty}\right)$ for some $\left(y^{\prime},-1\right) \in \mathrm{Cyl}_{-1}$, again for large $j$. If $\bar{\mu}$ is the constant from Lemma 6.8 , and $\mu_{1}<\bar{\mu}$, then that lemma gives a contradiction.

Proof of Theorem 6.1. This follows by Proposition 6.10 and Lemma 6.11.

\section{Finiteness of points with bad worldlines}

In this section we study bad worldlines; recall from Definition 1.9 that a worldline $\gamma: I \rightarrow$ $\mathcal{M}$ in a singular Ricci flow $\mathcal{M}$ is bad if inf $I>0$. In Theorem 7.1 we prove that only finitely many bad worldlines intersect a given connected component in a given time slice. We then give some applications.

The main result of this section is the following theorem.

THEOREM 7.1. Let $\mathcal{M}$ be a singular Ricci flow. For $T<\infty$, every connected component $C_{T}$ of $\mathcal{M}_{T}$ intersects at most $N$ bad worldlines, where $N=N\left(T, \operatorname{vol}\left(\mathcal{M}_{0}\right)\right)$. In particular, the set of bad worldlines in $\mathcal{M}$ is at most countable. Moreover, if $\gamma: I \rightarrow \mathcal{M}$ is a bad worldline then, for $t \in I$ sufficiently close to inf $I, \gamma(t)$ lies in a cap region of $\mathcal{M}_{t}$.

We begin with a lemma. 
Lemma 7.2. For all $D<\infty$ there exist $\hat{\varepsilon}=\hat{\varepsilon}(\varkappa, D)>0$ and $A=A(\varkappa, D)<\infty$ such that, if $m \in \mathcal{M}_{t}$ and $(\mathcal{M}, m)$ is $\hat{\varepsilon}$-modelled (see $\left.\S \mathrm{A} .2\right)$ on a $\varkappa$-solution of diameter $\leqslant D$, then the following statements hold.

(1) The connected component $N_{t}$ of $\mathcal{M}_{t}$ containing $m$ is compact and has $\mathrm{Rm}>0$.

(2) Let $g^{\prime}(\cdot)$ be the Ricci flow on the (smooth manifold underlying) $N_{t}$ defined on the time interval $\left[t, T^{\prime}\right)$, with initial condition at time $t$ given by $g^{\prime}(t)=\left.g\right|_{N_{t}}$, and blow-up time $T^{\prime}$. Let $\mathcal{N}$ be the corresponding Ricci flow spacetime. Then $\mathcal{N}_{\leqslant t^{\prime}}$ is compact for all $t^{\prime}<T^{\prime}$, and if $\tilde{\mathcal{M}}$ is the connected component of $\mathcal{M}_{\geqslant t}$ containing $N_{t}$, then $\widetilde{\mathcal{M}}$ is isomorphic to $\mathcal{N}$.

(3) $R \geqslant A R(m)$ on $\tilde{\mathcal{M}}$.

Proof. (1) Let $\mathrm{Sol}_{\varkappa}^{\mathrm{D}}$ be the collection of pointed $\varkappa$-solutions $\left(\mathcal{M}^{\prime},(x, 0)\right)$ such that $R(x, 0)=1$, and $\operatorname{Diam}\left(\mathcal{M}_{0}^{\prime}\right) \leqslant D$. Then every $\left(\mathcal{M}^{\prime},(x, 0)\right) \in \operatorname{Sol}_{\varkappa}^{\mathrm{D}}$ has $\mathrm{Rm}>0$, and since $\mathrm{Sol}_{\varkappa}^{\mathrm{D}}$ is compact, there is a $\lambda>0$ such that $\mathrm{Rm} \geqslant \lambda$ in $\mathcal{M}_{0}^{\prime}$ for all $\left(\mathcal{M}^{\prime},(x, 0)\right) \in \operatorname{Sol}_{\varkappa}^{\mathrm{D}}$. Part (1) now follows.

(2) Let $\mathcal{N}$ be as above. Then $\mathcal{N}_{\left[t, t^{\prime}\right]}$ is compact for all $t^{\prime} \in\left[t, T^{\prime}\right)$, and by Hamilton's theorem for manifolds with Ric $>0$, we know that

$$
\min _{m^{\prime} \in \mathcal{N}_{t^{\prime}}} R\left(m^{\prime}\right) \rightarrow \infty \quad \text { as } t^{\prime} \rightarrow T^{\prime}
$$

Consider an isometric embedding of Ricci flow spacetimes $\mathcal{N}_{J} \hookrightarrow \mathcal{M}_{J}$ that extends the isometric embedding $\mathcal{N}_{t} \rightarrow \mathcal{M}_{t}$, and which is defined on a maximal time interval $J$ starting at time $t$. Notice that $J$ cannot be a closed interval $[t, \hat{t}]$, since then the embedding could be extended to a larger time interval using uniqueness for Ricci flows. If $J=[t, \hat{t})$ with $\hat{t}<T^{\prime}$, then, since $R$ is bounded on $\mathcal{N}_{[t, \hat{t}]}$, by the properness of $R$ on $\mathcal{M}_{\leqslant T^{\prime}}$ we may extend the embedding to an isometric embedding $\mathcal{N}_{[t, \hat{t}]} \rightarrow \mathcal{M}_{[t, \hat{t}]}$, contradicting the maximality of $J$. Therefore, there exists an isometric embedding $\mathcal{N}_{\left[t, T^{\prime}\right)} \rightarrow \mathcal{M}_{\left[t, T^{\prime}\right)}$ of Ricci flow spacetimes, as asserted. The image is clearly an open subset of $\mathcal{M}_{\geqslant t}$; it is closed, by (7.3). This proves (2).

(3) From the proof of (1) above, for every $\left(\mathcal{M}^{\prime},(x, 0)\right) \in \operatorname{Sol}_{\varkappa}^{\mathrm{D}}$ we have $R \geqslant 6 \lambda$ on $\mathcal{M}_{0}^{\prime}$. Taking $A=3 \lambda$, and $\hat{\varepsilon}$ sufficiently small, we get that $R \geqslant A R(m)$ in $N_{t}$, and therefore in $\mathcal{N}_{t}$ as well. By the maximum principle applied to the scalar curvature evolution equation, we have $R \geqslant A R(m)$ on $\mathcal{N}$, and hence on $\widetilde{\mathcal{M}}$ as well.

Proof of Theorem 7.1. In the proof below, we take $\varkappa=\varkappa(T)$. Recall that $\varepsilon$ is the global parameter used in the definition of a Ricci flow spacetime from Definition 1.4.

Let $\varepsilon_{1}, \Delta>0$ be constants, to be determined later. During the course of the argument below, we will state a number of inequalities involving $\varepsilon_{1}$ and $\Delta$; these will be treated as a cumulative set of constraints imposed on $\varepsilon_{1}$ and $\Delta$, i.e. we will be assuming that each inequality is satisfied. 
We recall that, by Proposition 5.17, $C_{T}$ determines a connected component $C_{t}$ of $\mathcal{M}_{t}$ for all $t \leqslant T$. Let Bad be the collection of bad worldlines intersecting $C_{T}$.

Choose $0<t_{-}<t_{+} \leqslant T$ such that $t_{+}-t_{-}<\Delta$, and let $\operatorname{Bad}_{\left[t_{-}, t_{+}\right)}$be the set of worldlines $\gamma: I \rightarrow \mathcal{M}$ which belong to Bad, where inf $I \in\left[t_{-}, t_{+}\right)$. We will show that, if $t_{+}-t_{-}<\Delta=$ $\Delta(\varkappa, T, \varepsilon)$, then $\left|\operatorname{Bad}_{\left[t_{-}, t_{+}\right)}\right|$is bounded by a function of $\varkappa, T$ and $\operatorname{vol}\left(\mathcal{M}_{0}\right)$; the theorem then follows immediately.

Step 1. If $\Delta<\bar{\Delta}\left(\varepsilon_{1}, \varkappa, \varepsilon, T\right)$, then there exists $\widehat{T} \in[0, T]$ such that

(a) for every worldline $\gamma: I \rightarrow \mathcal{M}$ in Bad we have inf $I<\widehat{T}$;

(b) for every worldline $\gamma: I \rightarrow \mathcal{M}$ in $\operatorname{Bad}_{\left[t_{-}, t_{+}\right)}$, and every $t \in\left(\inf I, t_{+}\right]$with $t \leqslant \widehat{T}$, the pair $(\mathcal{M}, \gamma(t))$ is $\varepsilon_{1}$-modelled on a non-compact $\varkappa$-solution.

By the compactness of the space of normalized pointed $\varkappa$-solutions (see $\S$ A.5), there exist $\varepsilon_{2}=\varepsilon_{2}\left(\varepsilon_{1}\right)>0$ and $D=D\left(\varepsilon_{1}\right)<\infty$ such that, if $(\mathcal{M}, \gamma(t))$ is $\varepsilon_{2}$-modelled on a pointed $\varkappa$-solution with diameter greater than $D$, then it is $\frac{1}{2} \varepsilon_{1}$-modelled on a non-compact $\varkappa$-solution. Put $\varepsilon_{3}=\frac{1}{2} \min \left(\hat{\varepsilon}(\varkappa, D), \varepsilon_{2}\right)$, where $\hat{\varepsilon}(\varkappa, D)$ is as in Lemma 7.2.

Let $\mathcal{W}$ be the set of $m \in \bigcup_{t \leqslant T} C_{t}$ such that the pair $(\mathcal{M}, m)$ is $\varepsilon_{3}$-modelled on a pointed $\varkappa$-solution with diameter at most $D$. Suppose first that $\mathcal{W}$ is non-empty. Lemma 7.2 implies that $R(m) \leqslant A^{-1} \inf _{C_{T}} R$ for all $m \in \mathcal{W}$; therefore, by the properness of $R: \mathcal{M}_{\leqslant T} \rightarrow \mathbb{R}$, the time function $\mathfrak{t}$ attains a minimum value $\widehat{T}$ on $\mathcal{W}$. Pick $m \in \mathcal{W} \cap \mathcal{M}_{\widehat{T}}$. Then, by Lemma 7.2 , the connected component of $\mathcal{M}_{[\widehat{T}, T]}$ containing $m$ is isomorphic to the $[\widehat{T}, T]$-time slab of the spacetime $\mathcal{N}$ of a Ricci flow on a compact manifold with positive Ricci curvature. In particular, it also coincides with $\bigcup_{t \in[\widehat{T}, T]} C_{t}$, and therefore the curvature is bounded on the latter. Hence, for every $\gamma: I \rightarrow \mathcal{M}$ in Bad, we have $\inf I<\widehat{T}$. If $\mathcal{W}$ is empty, then we put $\widehat{T}=T$; then, the conclusion of part (a) of Step 1 still holds, so we continue.

By Proposition 5.15, there exists $\widehat{R}=\widehat{R}\left(\varepsilon_{3}, \varkappa, r(T)\right)$ such that, for all $m \in \mathcal{M}_{\leqslant T}$ with $R(m) \geqslant \widehat{R}$, the pair $(\mathcal{M}, m)$ is $\varepsilon_{3}$-modelled on a pointed $\varkappa$-solution. By Lemma 5.14, if $\Delta<\bar{\Delta}(\widehat{R}, \varkappa), \gamma: I \rightarrow \mathcal{M}$ belongs to $\operatorname{Bad}_{\left[t_{-}, t_{+}\right)}$and $t \in\left[t_{-}, t_{+}\right] \cap I \cap[0, \widehat{T})$, then we have $R(\gamma(t))>\widehat{R}$. Hence, either $(M, \gamma(t))$ is $(\mathrm{A}) \frac{1}{2} \varepsilon_{1}$-modelled on a non-compact $\varkappa$-solution, or (B) $\varepsilon_{3}$-modelled on a $\varkappa$-solution of diameter at most $D$; but in case (B) we would have $\gamma(t) \in \mathcal{W}$, which is impossible because $t<\widehat{T}$. This proves that part (b) of Step 1 holds when $t<\widehat{T}$, with $\varepsilon_{1}$ replaced by $\frac{1}{2} \varepsilon_{1}$ in the statement. The borderline case $t=\widehat{T}$ now follows by applying the previous arguments to times $t$ slightly less than $\widehat{T}$ and taking the limit as $t \nearrow \widehat{T}$. This completes Step 1 .

Hereafter, we assume that $\Delta<\bar{\Delta}\left(\varepsilon_{1}, \varkappa, \varepsilon, T\right)$. By part (a) of Step 1, the set Bad is the same as the set of bad worldlines intersecting $C_{\widehat{T}}$. Hence, we may replace $T$ by $\widehat{T}$; then, by part (b) of Step 1 , for every $\gamma: I \rightarrow \mathcal{M}$ in $\operatorname{Bad}_{\left[t_{-}, t_{+}\right)}$and every $t \in\left[t_{-}, t_{+}\right]$, the pair 
$(\mathcal{M}, \gamma(t))$ is $\varepsilon_{1}$-modelled on a non-compact $\varkappa$-solution.

Step 2. Provided $\varepsilon_{1}<\varepsilon_{1}(\varkappa)$, for all $\gamma: I \rightarrow \mathcal{M}$ belonging to $\operatorname{Bad}_{\left[t_{-}, t_{+}\right)}$and every $t \in I \cap\left[t_{-}, t_{+}\right)$, the pair $(\mathcal{M}, \gamma(t))$ is not a generalized $\frac{1}{2} \delta_{\text {neck-neck. }}$ (Here, $\delta_{\text {neck }}$ is the parameter from Corollary 6.2, and a generalized neck is in the sense of $\S$ A.2.)

Suppose that $\gamma: I \rightarrow \mathcal{M}$ belongs to $\operatorname{Bad}_{\left[t_{-}, t_{+}\right)}$, and $\left(\mathcal{M}, \gamma\left(\hat{t}_{0}\right)\right)$ is a generalized $\frac{1}{2} \delta_{\text {neck }^{-}}$ neck for some $\hat{t}_{0} \in I \cap\left[t_{-}, t_{+}\right)$. By Step 1 , we know that $\left(\mathcal{M}, \gamma\left(\hat{t}_{0}\right)\right)$ is $\varepsilon_{1}$-modelled on a non-compact pointed $\varkappa$-solution $\left(\mathcal{M}^{1},\left(x_{0}, 0\right)\right)$. If $\varepsilon_{1}<\bar{\varepsilon}_{1}\left(\frac{1}{2} \delta_{\text {neck }}\right)$, then $\left(\mathcal{M}^{1},\left(x_{0}, 0\right)\right)$ will

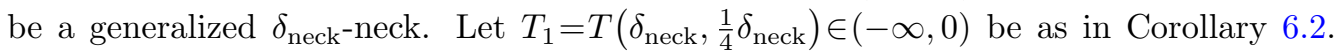
Then, $\left(\mathcal{M}^{1},\left(x_{0}, T_{1}\right)\right)$ is a generalized $\frac{1}{4} \delta_{\text {neck-neck. If }} \varepsilon_{1}<\bar{\varepsilon}_{1}\left(T_{1}, \frac{1}{2} \delta_{\text {neck }}\right)$, then we get that

- $\gamma$ is defined at $\hat{t}_{1}=\hat{t}_{0}+R^{-1}\left(\gamma\left(\hat{t}_{0}\right)\right) T_{1}$;

- $\left(\mathcal{M}, \gamma\left(\hat{t}_{1}\right)\right)$ is a generalized $\frac{1}{2} \delta_{\text {neck-neck; }}$

- $R\left(\gamma\left(\hat{t}_{1}\right)\right)<\frac{1}{2} R\left(\gamma\left(\hat{t}_{0}\right)\right)$.

Thus, we may iterate this to produce a sequence $\left\{\hat{t}_{0}, \hat{t}_{1}, \ldots\right\} \subset I$ such that

$$
\hat{t}_{i} \leqslant \hat{t}_{i-1}+R^{-1}\left(\gamma\left(\hat{t}_{0}\right)\right) T_{1} \text { for all } i \text {. }
$$

This contradicts the fact that inf $I \in\left[t_{-}, t_{+}\right)$, and completes Step 2 .

Hereafter, we assume that $\varepsilon_{1}<\varepsilon_{1}(\varkappa)$. Let $D_{0}<\infty$ be such that, if $\mathcal{M}^{\prime}$ is a noncompact $\varkappa$-solution, $m_{1}, m_{2} \in \mathcal{M}_{t}^{\prime}$ and neither $m_{1}$ nor $m_{2}$ is a $\frac{1}{4} \delta_{\text {neck-neck, then }}$

$$
d_{t}\left(m_{1}, m_{2}\right)<D_{0} R\left(m_{1}\right)^{-1 / 2} .
$$

Let $D_{1} \in\left(2 D_{0}, \infty\right)$ be a constant, to be determined in Step 4 .

Step 3. Provided $\varepsilon_{1}<\bar{\varepsilon}_{1}^{\prime}\left(\varkappa, D_{1}\right)$, if $\gamma_{1}, \gamma_{2} \in \operatorname{Bad}_{\left[t_{-}, t_{+}\right)}$and

$$
\left.d_{t_{+}}\left(\gamma_{1}\left(t_{+}\right), \gamma_{2}\left(t_{+}\right)\right)<D_{1} R\left(\gamma_{1}\left(t_{+}\right)\right)\right)^{-1 / 2},
$$

then $\gamma_{1}=\gamma_{2}$.

Let $I_{i}$ be the domain of $\gamma_{i}$, for $i \in\{1,2\}$. Suppose that $t \in I_{1} \cap I_{2} \cap\left[t_{-}, t_{+}\right]$. By Steps 1 and 2, $\left(\mathcal{M}, \gamma_{1}(t)\right)$ is $\varepsilon_{1}$-modelled on a non-compact $\varkappa$-solution, and neither $\gamma_{1}(t)$ nor $\gamma_{2}(t)$ is a $\frac{1}{2} \delta_{\text {neck }}$-neck. If $\varepsilon_{1}<\bar{\varepsilon}_{1}^{\prime}\left(D_{1}, \delta_{\text {neck }}\right)$ then, using the $\varepsilon_{1}$-closeness to a non-compact $\varkappa$-solution and (7.4), we can say that

$$
\left.d_{t}\left(\gamma_{1}(t), \gamma_{2}(t)\right)<D_{1} R\left(\gamma_{1}(t)\right)\right)^{-1 / 2}
$$

implies

$$
\left.d_{t}\left(\gamma_{1}(t), \gamma_{2}(t)\right)<2 D_{0} R\left(\gamma_{1}(t)\right)\right)^{-1 / 2}
$$


Since we are assuming (7.5), a continuity argument shows that (7.6) holds for all $t \in I_{1} \cap I_{2} \cap\left[t_{+}, t_{-}\right]$. If $\inf I_{1} \geqslant \inf I_{2}$, then

$$
\lim _{t \rightarrow \inf I_{1}} R\left(\gamma_{2}(t)\right)=\infty
$$

so inf $I_{1}=\inf I_{2}$; a similar reasoning holds if inf $I_{2} \leqslant \inf I_{1}$. Thus, inf $I_{1}=\inf I_{2}$. Moreover, if $\varepsilon_{1}<\bar{\varepsilon}_{1}^{\prime \prime}(\varkappa)$, then any geodesic from $\gamma_{1}(t)$ to $\gamma_{2}(t)$ in $C_{t}$ will lie in the set with Ric $>0$, so $d_{t}\left(\gamma_{1}(t), \gamma_{2}(t)\right)$ is a decreasing function of $t$. Since (7.6) implies that $d_{t}\left(\gamma_{1}(t), \gamma_{2}(t)\right) \rightarrow 0$ as $t \rightarrow \inf I_{1}$, it follows that $\gamma_{1}=\gamma_{2}$. This completes Step 3 .

Hereafter, we assume that $\varepsilon_{1}<\bar{\varepsilon}_{1}^{\prime}\left(\varkappa, D_{1}\right)$.

Step 4. Provided $\Delta<\bar{\Delta}(\varkappa, T)$, the cardinality of $\operatorname{Bad}_{\left[t_{-}, t_{+}\right)}$is at most

$$
N=N\left(\varkappa, T, \operatorname{vol}\left(\mathcal{M}_{0}\right)\right) .
$$

Take $\hat{\varepsilon}=\frac{1}{2} \delta_{\text {neck }}$, and let $C_{1}=C_{1}(\hat{\varepsilon}, T), \bar{R}=\bar{R}(\hat{\varepsilon}, T)$, and $N_{1}, \ldots, N_{k} \subset \mathcal{M}_{t_{+}}$be as in Proposition 5.16. With reference to Step 3, take $D_{1}=C_{1}$. Let $\Delta<\bar{\Delta}(\bar{R}, \varkappa, r(T))$ be such that if $\gamma: I \rightarrow \mathcal{M}$ belongs to $\operatorname{Bad}_{\left[t_{-}, t_{+}\right)}$, and $t \in\left[t_{-}, t_{+}\right] \cap I$, then $R(\gamma(t)) \geqslant \bar{R}$; cf. the proof of Step 1.

Then the set

$$
S=\left\{\gamma\left(t_{+}\right): \gamma \in \operatorname{Bad}_{\left[t_{-}, t_{+}\right)}\right\}
$$

is contained in

$$
\left\{m \in \mathcal{M}_{t_{+}}: R(m) \geqslant \bar{R}\right\} \subset \bigcup_{i} N_{i} .
$$

By Step 3, for any two distinct elements $\gamma_{1}, \gamma_{2} \in \operatorname{Bad}_{\left[t_{-}, t_{+}\right)}$we have

$$
d_{t}\left(\gamma_{1}\left(t_{+}\right), \gamma_{2}\left(t_{+}\right)\right) \geqslant D_{1} R^{-1 / 2}\left(\gamma_{1}\left(t_{+}\right)\right)=C_{1} R^{-1 / 2}\left(\gamma_{1}\left(t_{+}\right)\right) .
$$

By Proposition 5.16, for all $i \in\{1, \ldots, k\}$ we have $\left|S \cap N_{i}\right| \leqslant 2$. Therefore, $|S| \leqslant 2 k$. This proves that $\operatorname{Bad}_{\left[t_{-}, t_{+}\right)}$is finite, and hence a weaker version of the theorem, namely that the set of all bad worldlines is countable.

Since the set of bad worldlines is countable, their union has measure zero in spacetime. Therefore, we may apply Proposition 5.5 to conclude that

$$
\operatorname{vol}\left(C_{t+}\right) \leqslant \operatorname{vol}\left(\mathcal{M}_{t+}\right) \leqslant \mathcal{V}(0)(1+2 t)^{3 / 2} .
$$

If $k \geqslant 2$, then each $N_{i}$ has non-empty boundary. Hence, Proposition $5.16(5)$ gives a bound

$$
k<k(\mathcal{V}(0), T) .
$$

This proves Theorem 7.1; the assertion that bad worldlines are confined to cap regions was established in the proof above. 
COROllary 7.7. If $\mathcal{M}$ is a singular Ricci flow, then the conclusions of Proposition 5.5 hold.

Proof. By Theorem 7.1, the set of bad worldlines is countable, and hence has measure zero. Combining this with Lemma 5.13 shows that the hypotheses of Proposition 5.5 are satisfied.

Remark 7.8. For mean-convex mean-curvature flow, the continuity of the volume follows from the weak continuity of the mass measure, as established in [53].

Theorem 7.1 also has the following topological implications.

Corollary 7.9. Let $\mathcal{M}$ be a singular Ricci flow.

(1) If $T \geqslant 0$ and $W \subset \mathcal{M}_{T}$ is an open subset that does not contain any compact connected components of $\mathcal{M}_{T}$, then there is a smooth time-preserving map

$$
\Gamma: W \times[0, T] \longrightarrow \mathcal{M}
$$

that is a "weak isotopy", in the sense that it maps $W \times\{t\}$ diffeomorphically onto an open subset of $\mathcal{M}_{t}$, for all $t \in[0, T]$.

(2) For all $T \geqslant 0$, the pair $\left(\mathcal{M}, \mathcal{M}_{\leqslant T}\right)$ is $k$-connected for $k \leqslant 2$.

Proof. (1) Let $\mathcal{C}$ be the collection of connected components of $\mathcal{M}_{T}$. Pick $C \in \mathcal{C}$. Let $B$ be the set of bad worldlines intersecting $C$. By Theorem 7.1 , the set $B$ is finite, so its intersection with $C$ is contained in a 3 -disk $D^{3}$. There is a $t_{C}<T$ such that the worldline of every $m \in C$ is defined in the interval $\left[t_{C}, T\right]$. Hence, we get a time-preserving map $F_{C}: C \times\left[t_{C}, T\right] \rightarrow \mathcal{M}$ that is a diffeomorphism onto its image.

By assumption, either $C$ is non-compact, or $C$ is compact and $W \not \supset C$. Therefore, there is a smooth homotopy $\left\{H_{t}: W \cap C \rightarrow C\right\}_{t \in\left[t_{C}, T\right]}$ (purely in the time- $T$ slice) such that $H_{T}: W \cap C \rightarrow C$ is the inclusion map, $H_{t}: W \cap C \rightarrow C$ is a diffeomorphism onto its image for all $t \in\left[t_{C}, T\right]$, and $H_{t_{C}}(W \cap C) \cap D^{3}=\varnothing$. We define $\Gamma$ on $(W \cap C) \times\left[t_{C}, T\right]$ by

$$
\Gamma(m, t)=F_{C}\left(H_{t}(m), t\right),
$$

and extend this to $(W \cap C) \times\left[0, t_{C}\right]$ by following worldlines. Note that, if $C_{1}, C_{2} \in \mathcal{C}$ are distinct components of $\mathcal{M}_{T}$, then $F_{C_{1}}\left(C_{1} \cap W\right)$ is disjoint from $F_{C_{2}}\left(C_{2} \cap W\right)$, so the resulting map $\Gamma$ has the property that $\Gamma(\cdot, t): W \rightarrow \mathcal{M}_{t}$ is an injective local diffeomorphism for every $t \in[0, T]$.

(2) Suppose that $0 \leqslant k \leqslant 2$ and $f:\left(D^{k}, \partial D^{k}\right) \rightarrow\left(\mathcal{M}, \mathcal{M}_{\leqslant T}\right)$ is a map of pairs, where $\partial D^{k}=S^{k-1}$ if $k \geqslant 1$ and $\partial D^{0}=\varnothing$. By Theorem 7.1, the Hausdorff dimension of the bad worldlines is at most 1 . Then, after making a small homotopy, we may assume that $f$ is smooth, and that its image is disjoint from the bad worldlines. We can now find a homotopy through maps of pairs by using the backward flow of the time vector field, which is well defined on $f\left(D^{k}\right)$. 


\section{Appendix A. Background material}

In this appendix we collect some needed facts about Ricci flows and Ricci flows with surgery. More information can be found in [44].

\section{A.1. Notation and terminology}

Let $\left(\mathcal{M}, \mathfrak{t}, \partial_{\mathfrak{t}}, g\right)$ be a Ricci flow spacetime (Definition 1.2). For brevity, we will often write $\mathcal{M}$ for the quadruple. In a Ricci flow with surgery, we will sometimes loosely write a point $m \in \mathcal{M}_{t}$ as a pair $(x, t)$.

Given $s>0$, the rescaled Ricci flow spacetime is $\widehat{\mathcal{M}}(s)=\left(\mathcal{M}, \mathfrak{t} / s, s \partial_{\mathfrak{t}}, g / s\right)$.

Given $m \in M_{t}$, we write $B(m, r)$ for the open metric ball of radius $r$ in $\mathcal{M}_{t}$. We write $P(m, r, \Delta t)$ for the parabolic neighborhood, i.e. the set of points $m^{\prime}$ in $\mathcal{M}_{[t, t+\Delta t]}$ if $\Delta t>0$ (or $\mathcal{M}_{[t+\Delta t, t]}$ if $\Delta t<0$ ) that lie on the worldline of some point in $B(m, r)$. We say that $P(m, r, \Delta t)$ is unscathed if $B(m, r)$ has compact closure in $\mathcal{M}_{t}$ and for every $m^{\prime} \in P(m, r, \Delta t)$ the maximal worldline $\gamma$ through $m^{\prime}$ is defined on a time interval containing $[t, t+\Delta t]$ (or $[t+\Delta t, t])$. We write $P_{+}(m, r)$ for the forward parabolic ball $P\left(m, r, r^{2}\right)$ and $P_{-}(m, r)$ for the backward parabolic ball $P\left(m, r,-r^{2}\right)$.

We write Cyl for the standard Ricci flow on $S^{2} \times \mathbb{R}$ that terminates at time zero, with $g(t)=(-2 t) g_{S^{2}}+d z^{2}$. We write Sphere for the standard round shrinking 3-sphere that terminates at time zero.

\section{A.2. Closeness of Ricci flow spacetimes}

Let $\mathcal{M}^{1}$ and $\mathcal{M}^{2}$ be two Ricci flow spacetimes in the sense of Definition 1.2. Consider a time interval $[a, b]$. Suppose that $m_{1} \in \mathcal{M}^{1}$ and $m_{2} \in \mathcal{M}^{2}$ have $\mathfrak{t}_{1}\left(m_{1}\right)=\mathfrak{t}_{2}\left(m_{2}\right)=b$. We say that $\left(\mathcal{M}^{2}, m_{2}\right)$ is $\varepsilon$-close to $\left(\mathcal{M}^{1}, m_{1}\right)$ on the time interval $[a, b]$, if there are open subsets $U_{i} \subset \mathcal{M}^{i}$ with $P\left(m_{i}, \varepsilon^{-1}, a-b\right) \subset U_{i}, i \in\{1,2\}$, and there is a pointed diffeomorphism $\Phi:\left(U_{1}, m_{1}\right) \rightarrow\left(U_{2}, m_{2}\right)$ such that

- $B\left(m_{i}, \varepsilon^{-1}\right)$ has compact closure in $\mathcal{M}_{b}^{i}$;

- $P\left(m_{i}, \varepsilon^{-1}, a-b\right)$ is unscathed;

- $\Phi$ is time preserving, i.e. $\mathfrak{t}_{2} \circ \Phi=\mathfrak{t}_{1}$;

- $\Phi_{*} \partial_{\mathfrak{t}_{1}}=\partial_{\mathrm{t}_{2}}$;

- $\Phi^{*} g_{2}-g_{1}$ has norm less than $\varepsilon$ in the $C^{\lfloor 1 / \varepsilon\rfloor+1}$-topology (as defined using $g_{1}$ ) on $U_{1}$ (where $\lfloor\cdot\rfloor$ denotes the integer part).

Remark A.1. The notion of $\varepsilon$-closeness is not symmetric with respect to $\left(\mathcal{M}^{1}, m_{1}\right)$ and $\left(\mathcal{M}^{2}, m_{2}\right)$, but this will not be an issue, since we only use the associated topology. 
Now, consider an open time interval $(-\infty, b)$. Suppose that

$$
\mathfrak{t}_{1}\left(m_{1}\right)=\mathfrak{t}_{2}\left(m_{2}\right)=c \in(-\infty, b)
$$

After time shift and parabolic rescaling, we may assume that $c=-1$ and $b=0$. In this case, we say that $\left(\mathcal{M}^{2}, m_{2}\right)$ is $\varepsilon$-close to $\left(\mathcal{M}^{1}, m_{1}\right)$ on the time interval $(-\infty, 0)$ if there are open sets $U_{i} \subset \mathcal{M}^{i}$ with $P\left(m_{i}, \varepsilon^{-1}, 1-\varepsilon\right) \cup P\left(m_{i}, \varepsilon^{-1},-\varepsilon^{-2}\right) \subset U_{i}, i \in\{1,2\}$, and there is a pointed diffeomorphism $\Phi:\left(U_{1}, m_{1}\right) \rightarrow\left(U_{2}, m_{2}\right)$ such that

(1) $B\left(m_{i}, \varepsilon^{-1}\right)$ has compact closure in $\mathcal{M}_{-1}^{i}$;

(2) $P\left(m_{i}, \varepsilon^{-1}, 1-\varepsilon\right)$ and $P\left(m_{i}, \varepsilon^{-1},-\varepsilon^{-2}\right)$ are unscathed;

(3) $\Phi$ is time preserving, i.e. $\mathfrak{t}_{2} \circ \Phi=\mathfrak{t}_{1}$;

(4) $\Phi_{*} \partial_{\mathfrak{t}_{1}}=\partial_{\mathfrak{t}_{2}}$

(5) $\Phi^{*} g_{2}-g_{1}$ has norm less than $\varepsilon$ in the $C^{\lfloor 1 / \varepsilon\rfloor+1}$-topology on $U_{1}$.

Let $\left(\mathcal{M}_{1}, m_{1}\right)$ and $\left(\mathcal{M}_{2}, m_{2}\right)$ be as above, and $u_{i}: \mathcal{M}_{i} \rightarrow \mathbb{R}$ be a continuous function for $i \in\{1,2\}$. Then, we say that $\left(\mathcal{M}_{2}, m_{2}, u_{2}\right)$ is $\varepsilon$-close to $\left(\mathcal{M}_{1}, m_{1}, u_{1}\right)$ if (1)-(5) above hold and, in addition,

$$
\sup \left\{u_{2} \circ \Phi(m)-u_{1}(m): m \in P\left(m_{1}, \varepsilon^{-1}, 1-\varepsilon\right) \cup P\left(m_{1}, \varepsilon^{-1},-\varepsilon^{-2}\right)\right\}<\varepsilon
$$

We will apply this notion when the $u_{i}$ 's are reduced distance functions.

We define $\varepsilon$-closeness similarly on other time intervals, whether open or half-open.

Let $\left(\mathcal{M}_{1}, m_{1}\right)$ and $\left(\mathcal{M}_{2}, m_{2}\right)$ be Ricci flow spacetimes. Then, we say that $\left(\mathcal{M}_{2}, m_{2}\right)$ is $\varepsilon$-modelled on $\left(\mathcal{M}_{1}, m_{1}\right)$ if, after shifts in the time parameters such that $\mathfrak{t}_{1}\left(m_{1}\right)=$ $\mathfrak{t}_{2}\left(m_{2}\right)=0$, and parabolic rescaling by $R\left(m_{1}\right)$ and $R\left(m_{2}\right)$ respectively, the resulting Ricci flow spacetimes are $\varepsilon$-close on the time interval $\left[-\varepsilon^{-1}, 0\right]$. (It is implicit in the definition that $R\left(m_{1}\right)>0$ and $R\left(m_{2}\right)>0$; this will be the case for us, since we are interested in modelling regions of high scalar curvature.) A point $m$ in a Ricci flow spacetime $\mathcal{M}$ is a generalized $\varepsilon$-neck if $(\mathcal{M}, m)$ is $\varepsilon$-modelled on $\left(\mathcal{M}^{\prime}, m^{\prime}\right)$, where $\mathcal{M}^{\prime}$ is either a shrinking round cylinder or the $\mathbb{Z}_{2}$-quotient of a shrinking round cylinder.

\section{A.3. Necks, horns and caps}

We say that $(\mathcal{M}, m)$ is a (strong) $\delta$-neck if, after time shifting and parabolic rescaling, it is $\delta$-close on the time interval $[-1,0]$ to the product Ricci flow which, at its final time, is isometric to the product of $\mathbb{R}$ with a round 2 -sphere of scalar curvature 1 . The basepoint is taken at time 0 . In this case, we also say that $m$ is the center of a $\delta$-neck.

If $I$ is an open interval, then a metric on an embedded copy of $S^{2} \times I$ in $\mathcal{M}_{t}$ such that each point is contained in an $\delta$-neck is called a $\delta$-tube (resp. $\delta$-horn, or double $\delta$ horn) if the scalar curvature stays bounded on both ends (resp. stays bounded on one 
end and tends to infinity on the other, or tends to infinity on both ends). (Our definition differs slightly from that in [44, Definition 58.2], where the definition is in terms of the " $\delta$-necks" of that paper, as opposed to the "strong $\delta$-necks" that we are using now.)

A metric on $B^{3}$ or $B^{3} \backslash \mathbb{R} P^{3}$ such that each point outside some compact set is contained in a $\delta$-neck is called a $\delta$-cap (resp. capped $\delta$-horn) if the scalar curvature stays bounded (resp. tends to infinity) on the end.

\section{A.4. $\varkappa$-non-collapsing}

Let $\mathcal{M}$ be an $(n+1)$-dimensional Ricci flow spacetime. Let $\varkappa$ : $[0, \infty) \rightarrow(0, \infty)$ be a decreasing function. We say that $\mathcal{M}$ is $\varkappa$-non-collapsed at scales below $\varepsilon$ if, for each $\varrho<\varepsilon$ and all $m \in \mathcal{M}$ with $\mathfrak{t}(m) \geqslant \varrho^{2}$, whenever $P\left(m, \varrho,-\varrho^{2}\right)$ is unscathed and $|\mathrm{Rm}| \leqslant \varrho^{-2}$ on $P\left(m, \varrho,-\varrho^{2}\right)$, then we also have $\operatorname{vol}(B(m, \varrho)) \geqslant \varkappa(\mathfrak{t}(m)) \varrho^{n}$. In the application to Ricci flow with surgery, $\varepsilon$ will be taken to be the global parameter.

We refer to $[44, \S 15]$ for the definitions of the $l$-function $l(m)$ and the reduced volume $\widetilde{V}(\tau)$. For notation, we recall that the $l$-function is defined in terms of $\mathcal{L}$-geodesics going backward in time from a basepoint $m^{\prime} \in \mathcal{M}$. The parameter $\tau$ is backward time from $m^{\prime}$, i.e. $\tau(m)=\mathfrak{t}\left(m^{\prime}\right)-\mathfrak{t}(m)$.

\section{A.5. $\varkappa$-solutions}

Given $\varkappa \in \mathbb{R}^{+}$, a $\varkappa$-solution $\mathcal{M}$ is a smooth Ricci flow solution defined on a time interval of the form $(-\infty, C)$ (or $(-\infty, C])$ such that the following properties are satisfied:

- the curvature is uniformly bounded on each compact time interval, and each time slice is complete;

- the curvature operator is non-negative and the scalar curvature is everywhere positive;

- the Ricci flow is $\varkappa$-non-collapsed at all scales.

We will sometimes talk about $\varkappa$-solutions without specifying $\varkappa$. Unless explicitly specified, it is understood that $C=0$. If $(\mathcal{M}, m)$ is a pointed $\varkappa$-solution, then we will sometimes understand it to be defined on the interval $(-\infty, \mathfrak{t}(m)]$.

Examples of $\varkappa$-solutions are $\mathrm{Cyl}$ and Sphere.

Any pointed $\varkappa$-solution $(\mathcal{M}, m)$ has an asymptotic soliton. It is obtained by constructing the $l$-function using $\mathcal{L}$-geodesics emanating backward from $m$. For any $t<\mathfrak{t}(m)$, there is some point $m_{t}^{\prime} \in \mathcal{M}_{t}$ where $l\left(m_{t}^{\prime}\right) \leqslant \frac{1}{2} n$. Put $\tau=\mathfrak{t}(m)-t$. Then, the parabolic rescaling $\left(\widehat{\mathcal{M}}(\tau), m_{t}^{\prime}\right)$ subconverges, as $\tau \rightarrow \infty$, to a non-flat gradient shrinking soliton called the asymptotic soliton [44, Proposition 39.1]. (In the cited reference, the conver- 
gence is shown on the (rescaled) time interval $\left[-1,-\frac{1}{2}\right]$, but using the estimates of $\S$ A.7 one easily gets pointed convergence on the time interval $(-\infty, 0)$.)

Hereafter, we suppose that the spacetime $\mathcal{M}$ of the $\varkappa$-solution is 4 -dimensional. A basic fact is that the space of pointed $\varkappa$-solutions $(\mathcal{M}, m)$, with $R(m)=1$, is compact [44, Theorem 46.1].

Given $\delta>0$, let $\mathcal{M}_{\delta}$ denote the points in $\mathcal{M}$ that are not centers of $\delta$-necks. We call these cap points. Put $\mathcal{M}_{t, \delta}=\mathcal{M}_{t} \cap \mathcal{M}_{\delta}$. From [44, Corollary 47.2], if $\delta$ is small enough then there is a $C=C(\delta, \varkappa)>0$ such that, if $\mathcal{M}_{t}$ is non-compact, then

- $\mathcal{M}_{t, \delta}$ is compact with $\operatorname{Diam}\left(\mathcal{M}_{t, \delta}\right) \leqslant C Q^{-1 / 2}$ and

- $C^{-1} Q \leqslant R(m) \leqslant C Q$ whenever $m \in M_{t, \delta}$,

where $Q=R\left(m^{\prime}\right)$ for some $m^{\prime} \in \partial M_{t, \delta}$.

If $\mathcal{M}$ is non-compact, and not a round shrinking cylinder, then $\mathcal{M}_{t, \delta} \neq \varnothing$. A version of the preceding paragraph that also holds for compact $\varkappa$-solutions can be found in [44, Corollary 48.1].

A compact $\varkappa$-solution is either a quotient of the round shrinking sphere, or is diffeomorphic to $S^{3}$ or $\mathbb{R P}^{3}[44$, Lemma 59.3].

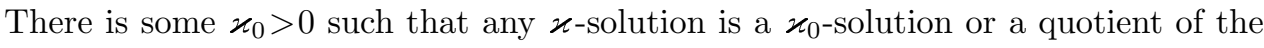
round shrinking $S^{3}$ [44, Proposition 50.1]

\section{A.6. Gradient shrinking solitons}

LEMMA A.2. Let $\mathcal{M}$ be a 3-dimensional gradient shrinking soliton that is a $\varkappa$ solution and blows up as $t \rightarrow 0$. For $t<0$ and a point $(y, t) \in \mathcal{M}$, let $l_{y, t} \in C^{\infty}\left(\mathcal{M}_{<t}\right)$ be the $l$-function on $\mathcal{M}$ constructed using $\mathcal{L}$-geodesics going backward in time from $(y, t)$. Then, there is a function $l_{\infty} \in C^{\infty}(\mathcal{M})$ such that the limit

$$
\lim _{t \rightarrow 0^{-}} l_{y, t}=l_{\infty}
$$

exists, independent of $y$, with continuous convergence on compact subsets on $\mathcal{M}$. Define $\widetilde{V}_{\infty}:(-\infty, 0) \rightarrow(0, \infty)$ as in $(6.5)$. Then, one of the following statements holds:

(1) $\mathcal{M}$ is the shrinking round cylinder solution $\mathrm{Cyl}$ on $S^{2} \times \mathbb{R}$, with

$$
R(x, t)=(-t)^{-1} \quad l_{\infty}((x, z), t)=1+\frac{z^{2}}{-4 t} \quad \text { and } \quad \widetilde{V}_{\infty}(t)=\frac{16 \pi^{3 / 2}}{e}
$$

(2) $\mathcal{M}$ is the $\mathbb{Z}_{2}$-quotient of the cylinder in $(2)$, with $\widetilde{V}_{\infty}(t)=8 \pi^{3 / 2} /$ e; the pull-back of $l_{\infty}$ to the cylinder is $1+z^{2} /(-4 t)$;

(3) $\mathcal{M}$ is a shrinking round spherical space form Sphere $/ \Gamma$, where $\Gamma \subset \mathrm{SO}(4)$,

$$
R(x, t)=\frac{3}{2}(-t)^{-1}, \quad l_{\infty}(x, t)=\frac{3}{2} \quad \text { and } \quad \tilde{V}_{\infty}(t)=\frac{16 \pi^{2} e^{-3 / 2}}{|\Gamma|} .
$$


Proof. The classification of the solitons follows from [44, Corollary 51.22]. Let $f$ be a potential for the soliton, i.e.

$$
\operatorname{Ric}+\operatorname{Hess}(f)=-\frac{1}{2 t} g
$$

and

$$
\frac{\partial f}{\partial t}=|\nabla f|^{2}
$$

There is a constant $C$ such that $R+|\nabla f|^{2}+f / t=-C / t$.

By [24, Theorem 3.7] and [56, Proposition 3.8], for any sequence $t_{i} \rightarrow 0^{-}$, after passing to a subsequence, there is a $\operatorname{limit}_{i \rightarrow \infty} \lim _{y, t_{i}}$ with continuous convergence on compact subsets of $\mathcal{M}$. In the case of a gradient shrinking soliton, [20, §7.7.3] implies that

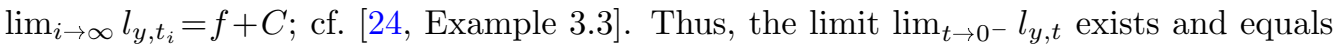
$f+C$, independent of $y$. In our case, the formulas for $l_{\infty}$ and $\widetilde{V}_{\infty}$ now follow from straightforward calculation.

\section{A.7. Estimates on $l$-functions}

We recall some estimates on the $l$-function that hold for $\varkappa$-solutions, taken from [81].

The letter $C$ will denote a generic universal constant. From [81, equation (2.53)],

$$
R \leqslant \frac{C l}{\tau}
$$

From [81, equations (2.54) and (2.56)],

$$
\max \left(|\nabla l|^{2},\left|l_{\tau}\right|\right) \leqslant \frac{C l}{\tau}
$$

From [81, equation (2.55)],

$$
\left|\sqrt{l}\left(q_{1}, \tau\right)-\sqrt{l}\left(q_{2}, \tau\right)\right| \leqslant \sqrt{\frac{C}{4 \tau}} d\left(q_{1}, q_{2}, \tau\right) .
$$

From [81, equation (2.57)],

$$
\left(\frac{\tau_{1}}{\tau_{2}}\right)^{C} \leqslant \frac{l\left(q, \tau_{2}\right)}{l\left(q, \tau_{1}\right)} \leqslant\left(\frac{\tau_{2}}{\tau_{1}}\right)^{C}
$$

From [81, equation (3.7)],

$$
-l\left(q_{1}, \tau\right)-1+C_{1} \frac{d^{2}\left(q_{1}, q_{2}, \tau\right)}{\tau} \leqslant l\left(q_{2}, \tau\right) \leqslant 2 l\left(q_{1}, \tau\right)+C_{2} \frac{d^{2}\left(q_{1}, q_{2}, \tau\right)}{\tau} .
$$




\section{A.8. Canonical neighborhoods}

In this subsection we recall the notion of a canonical neighborhood for a Ricci flow with surgery, and define the notion of a canonical neighborhood in a singular Ricci flow. We mention that this rather complicated looking definition is motivated by the structure of $\varkappa$-solutions and the standard (post-surgery) solution.

Let $r:[0, \infty) \rightarrow(0, \infty)$ be a decreasing function. Let $\varepsilon>0$ be small enough so that the bullet points at the end of $\S$ A.5 hold (with $\delta=\varepsilon$ ). Let $C_{1}=C_{1}(\varepsilon)$ and $C_{2}=C_{2}(\varepsilon)$ be the constants in [44, Definition 69.1].

As in [44, Definition 69.1], a Ricci flow with surgery $\mathcal{M}$ defined on the time interval $[a, b]$ satisfies the $r$-canonical neighborhood assumption if every $(x, t) \in \mathcal{M}_{t}^{ \pm}$with scalar curvature $R(x, t) \geqslant r(t)^{-2}$ has a canonical neighborhood in the corresponding forward/backward time slice, in the following sense: there is an

$$
\hat{r} \in\left(R(x, t)^{-1 / 2}, C_{1} R(x, t)^{-1 / 2}\right)
$$

and an open set $U \subset \mathcal{M}_{t}^{ \pm}$, with $\overline{B^{ \pm}(x, t, \hat{r})} \subset U \subset B^{ \pm}(x, t, 2 \hat{r})$, that falls into one of the following categories:

(a) $U \times[t-\Delta t, t] \subset \mathcal{M}$ is a strong $\varepsilon$-neck for some $\Delta t>0$ (note that, after parabolic rescaling, the scalar curvature at $(x, t)$ becomes 1 , so the scale factor must be $\approx R(x, t)$, which implies that $\left.\Delta t \approx R(x, t)^{-1}\right)$;

(b) $U$ is an $\varepsilon$-cap which, after rescaling, is $\varepsilon$-close to the corresponding piece of a $\varkappa_{0}$-solution or a time slice of a standard solution;

(c) $U$ is a closed manifold diffeomorphic to $S^{3}$ or $\mathbb{R P}^{3}$;

(d) $U$ is $\varepsilon$-close to a closed manifold of constant positive sectional curvature.

Moreover, the scalar curvature in $U$ lies between $C_{2}^{-1} R(x, t)$ and $C_{2} R(x, t)$. In cases (a)-(c), the volume of $U$ is greater than $C_{2}^{-1} R(x, t)^{-3 / 2}$. In case (c), the infimal sectional curvature of $U$ is greater than $C_{2}^{-1} R(x, t)$.

Finally, we require that

$$
|\nabla R(x, t)|<\eta R(x, t)^{3 / 2} \text { and } \quad\left|\frac{\partial R}{\partial t}(x, t)\right|<\eta R(x, t)^{2},
$$

where $\eta$ is the constant from [44, equation (59.5)]. Here, the time dervative

$$
\frac{\partial R}{\partial t}(x, t)
$$

should be interpreted as a one-sided derivative when the point $(x, t)$ is added or removed during surgery at time $t$.

We use a slightly simpler definition of canonical neighborhood in the case of singular Ricci flows, for Definition 1.4. We do not need to consider forward/backward time slices 
and, in case (b), we do not need to consider the case where $U$ is close to a time slice of a standard solution.

Remark A.9. Alternatively, for a singular Ricci flow, one could replace the above definition of canonical neighborhood with the requirement that every point satisfying $R \geqslant r(t)^{-2}$ is $\varepsilon$-modelled on a $\varkappa(t)$-solution. This is quantitatively equivalent to the definition above, as follows from Proposition 5.15 and [44, Lemma 59.7].

\section{A.9. Ricci flow with surgery}

We recall that there are certain parameters in the definition of Ricci flow with surgery, namely a number $\varepsilon>0$ and positive non-increasing functions $r, \varkappa, \delta:[0, \infty) \rightarrow(0, \infty)$. The function $r$ is the canonical neighborhood scale; cf. $\S$ A.8. The function $\varkappa$ is the noncollapsing parameter; cf. $\S$ A.4. The parameter $\varepsilon>0$ is a global parameter in the definition of a Ricci flow with surgery [44, Remark 58.5].

The function $\delta:[0, \infty) \rightarrow(0, \infty)$ is a surgery parameter. There is a further parameter $h(t)<\delta^{2}(t) r(t)$ such that, if a point $(x, t)$ lies in an $\varepsilon$-horn and has $R(x, t) \geqslant h(t)^{-2}$, then $(x, t)$ is the center of a $\delta(t)$-neck [44, Lemma 71.1]. One can then perform surgery on such cross-sectional 2-spheres [44, $\S 72$ and $\S 73]$. Perelman showed that there are positive non-increasing step functions $r_{P}, \varkappa_{P}$ and $\bar{\delta}_{P}$ such that, if the (positive non-increasing) function $\delta$ satisfies $\delta(t)<\bar{\delta}_{P}(t)$, then there is a well-defined Ricci flow with surgery, with a discrete set of surgery times [44, Sections 77-80].

In particular, we may assume that $\delta$ is strictly decreasing. If $r \leqslant r_{P}$ and $\varkappa \leqslant \varkappa_{P}$ are positive functions, then the $r_{P}$-canonical neighborhood assumption implies the $r$ canonical neighborhood assumption, and $\varkappa_{P}$-non-collapsing implies $\varkappa$-non-collapsing. Hence, Ricci flow with surgery also exists in terms of the parameters $(r, \varkappa, \delta)$. Consequently, we may assume that $r, \varkappa$ and $\delta$ are strictly decreasing.

Remark A.10. We remark that for the purposes of this paper, it is necessary to impose slightly stricter conditions on the surgery process than those that are needed for the proof of the geometrization conjecture. Specifically, in the definition of Ricci flow with $(r, \delta)$-cutoff [44, Definition 73.1], the surgery procedure involves choosing $\delta$-necks inside $\varepsilon$-horns, cutting along cross-sectional 2-spheres of the $\delta$-necks, and discarding the tips of the $\varepsilon$-horns; see steps (B)-(D) in [44, Definition 73.1]. Here, we require that the $\delta$-necks are chosen so that the discarded tips have scalar curvature at least $\frac{1}{100} h^{-2}$.

As in $[44, \S 68]$, the formal structure of a Ricci flow with surgery is given by the following data:

- a collection of Ricci flows $\left\{\left(M_{k} \times\left[t_{k}^{-}, t_{k}^{+}\right), g_{k}(\cdot)\right)\right\}_{1 \leqslant k \leqslant N}$, where $N \leqslant \infty, M_{k}$ is a 
compact (possibly empty) manifold, $t_{k}^{+}=t_{k+1}^{-}$for all $1 \leqslant k<N$, and the flow $g_{k}$ goes singular at $t_{k}^{+}$for each $k<N$; we allow $t_{N}^{+}$to be $\infty$;

- a collection of limits $\left\{\left(\Omega_{k}, \bar{g}_{k}\right)\right\}_{1 \leqslant k \leqslant N}$, in the sense of [44, $\left.\S 67\right]$, at the respective final times $t_{k}^{+}$that are singular if $k<N$ (here $\Omega_{k}$ is an open subset of $M_{k}$ );

- a collection of isometric embeddings $\left\{\psi_{k}: X_{k}^{+} \rightarrow X_{k+1}^{-}\right\}_{1 \leqslant k<N}$, where $X_{k}^{+} \subset \Omega_{k}$ and $X_{k+1}^{-} \subset M_{k+1}, 1 \leqslant k<N$, are compact 3-dimensional submanifolds with boundary. The $X_{k}^{ \pm}$'s are the subsets which survive the transition from one flow to the next, and the $\psi_{k}$ 's give the identifications between them.

We will say that $t$ is a singular time if $t=t_{k}^{+}=t_{k+1}^{-}$for some $1 \leqslant k<N$, or $t=t_{N}^{+}$and the metric goes singular at time $t_{N}^{+}$.

A Ricci flow with surgery need not have any real surgeries, i.e. it could be a smooth non-singular flow.

We now describe the Ricci flow spacetime associated with a Ricci flow with surgery. We mention that in [44] a spacetime object was associated with a Ricci flow with surgery; that construction was slightly different from the one used here, and did not produce a Ricci flow spacetime in the sense of Definition 1.2. We begin with the time slab $M_{k} \times\left[t_{k}^{-}, t_{k}^{+}\right]$for $1 \leqslant k \leqslant N$, which has a time function $\mathrm{t}: M_{k} \times\left[t_{k}^{-}, t_{k}^{+}\right] \rightarrow\left[t_{k}^{-}, t_{k}^{+}\right]$given by the projection onto the second factor, and a time vector field $\partial_{t}$ inherited from the coordinate vector field on the factor $\left[t_{k}^{-}, t_{k}^{+}\right]$.

For every $1<k \leqslant N$, put

$$
W_{k}^{-}=\left(M_{k} \backslash \operatorname{Int}\left(X_{k}^{-}\right)\right) \times\left\{t_{k}^{-}\right\}
$$

and, for $1 \leqslant k<N$, let

$$
W_{k}^{+}=\left(M_{k} \backslash \operatorname{Int}\left(X_{k}^{+}\right)\right) \times\left\{t_{k}^{+}\right\} .
$$

Since $W_{k}^{ \pm}$is a closed subset of the 4-manifold with boundary $M_{k} \times\left[t_{k}^{-}, t_{k}^{+}\right]$, the complement $Z_{k}=\left(M_{k} \times\left[t_{k}^{-}, t_{k}^{+}\right]\right) \backslash\left(W_{k}^{-} \cup W_{k}^{+}\right)$is a 4-manifold with boundary, where

$$
\partial Z_{k}=\left(M_{k} \times\left\{t_{k}^{-}, t_{k}^{+}\right\}\right) \backslash\left(W_{k}^{-} \cup W_{k}^{+}\right) .
$$

Note that the Ricci flow $g_{k}(\cdot)$ with singular limit $\bar{g}_{k}$ defines a smooth metric $\hat{g}_{k}$ on the sub-bundle ker $d \mathfrak{t} \subset T Z_{k}$ that satisfies $\mathcal{L}_{\partial_{\mathfrak{t}}} \hat{g}_{k}=-2 \operatorname{Ric}\left(\hat{g}_{k}\right)$.

For every $1 \leqslant k<N$ we glue $Z_{k}$ to $Z_{k+1}$ using the identification

$$
\operatorname{Int}\left(X_{k}^{+}\right) \stackrel{\psi_{k}}{\longrightarrow} \operatorname{Int}\left(X_{k+1}^{-}\right),
$$

to obtain a smooth 4-manifold with boundary $\mathcal{M}$, where $\partial \mathcal{M}$ is the image of $W_{1}^{-} \cup W_{N}^{+}$ under the quotient map $\bigsqcup_{k} Z_{k} \rightarrow \mathcal{M}$. The time functions, time vector fields and metrics 
descend to $\mathcal{M}$, yielding a tuple $\left(\mathcal{M}, \mathfrak{t}, \partial_{\mathfrak{t}}, g\right)$ which is a Ricci flow spacetime in the sense of Definition 1.2.

Recall the notion of a normalized Riemannian manifold from the introduction. Our convention is that the trace of the curvature operator is the scalar curvature. From [44, Appendix B], if a smooth 3-dimensional Ricci flow $\mathcal{M}$ has normalized initial condition, then the scalar curvature satisfies

$$
R(x, t) \geqslant-\frac{3}{1+2 t}
$$

If follows that the volume satisfies

$$
\mathcal{V}(t) \leqslant(1+2 t)^{3 / 2} \mathcal{V}(0)
$$

These estimates also hold for a Ricci flow with surgery.

Let $A$ be a symmetric $3 \times 3$ real matrix. Let $\lambda_{1}$ denote its smallest eigenvalue. For $t \geqslant 0$, put

$$
\begin{gathered}
K(t)=\left\{A: \operatorname{tr}(A) \geqslant-3 /(1+t) \text { and, if } \lambda_{1} \leqslant-1 /(1+t),\right. \text { then } \\
\left.\operatorname{tr}(A) \geqslant-\lambda_{1}\left(\log \left(-\lambda_{1}\right)+\log (1+t)-3\right)\right\} .
\end{gathered}
$$

Then $\left\{K(t\}_{t \geqslant 0}\right.$ is a family of $O(3)$-invariant convex sets which is preserved by the ODE on the space of curvature operators [21, proof of Theorem 6.44]. If a smooth 3-dimensional Ricci flow has normalized initial conditions, then the time-zero curvature operators lie in $K(0)$. Using (A.11), we obtain the Hamilton-Ivey estimate, according to which, whenever the lowest eigenvalue $\lambda_{1}(x, t)$ of the curvature operator satisfies $\lambda_{1} \leqslant-1 /(1+t)$, we have

$$
R \geqslant-\lambda_{1}\left(\log \left(-\lambda_{1}\right)+\log (1+t)-3\right)
$$

The surgery procedure is designed to ensure that (A.14) also holds for Ricci flows with surgery.

(Perelman's definition of a normalized Riemannian manifold is slightly different; he requires that the sectional curvatures be bounded by 1 in absolute value $[58, \S 5.1]$. With his convention, $R(x, t) \geqslant-6 /(1+4 t)$ and $\mathcal{V}(t) \leqslant(1+4 t)^{3 / 2} \mathcal{V}(0)$.)

\section{Appendix B. Extension of Proposition 6.10}

The main result in this appendix is Proposition B.1, which is an extension of Proposition 6.10 to general $\varkappa$-solutions. It uses similar ideas as Proposition 6.10 , but is a bit more complicated to state. We include this for the sake of completeness, as it gives a 
general quantitative picture of the behavior of $\varkappa$-solitons over time. It is not needed in the body of the paper.

Let $\mathcal{M}$ be a $\varkappa$-solution defined on $(-\infty, 0]$, and $l: \mathcal{M}_{<0} \rightarrow \mathbb{R}$ be the $l$-function with spacetime basepoint $(x, 0) \in \mathcal{M}_{0}$. Roughly speaking, Proposition B.1 says that the reduced volume for a $\varkappa$-solution, as a function of time, has two types of behavior: it can remain close to the reduced volume of a gradient shrinking soliton over a long time interval, or it can transition relatively quickly between two such values. During long time intervals of the former kind, the $\varkappa$-solution and $l$-function are close, modulo parabolic scaling, to a gradient shrinking soliton and its $l$-function. We now give the details.

Fix $\varkappa>0$. By Lemma A.2, there are only finitely many 3-dimensional gradient shrinking solitons that are $\varkappa$-solutions. Let $\mathcal{V}_{\text {sol }}=\left\{0<\widetilde{V}_{1}<\ldots<\widetilde{V}_{K}\right\}$ be the set of their reduced volumes, in the sense of Lemma A.2.

Proposition B.1. For all $\hat{\varepsilon}>0$ and $C<\infty$ there is a $\varrho=\varrho(\hat{\varepsilon}, C)<\infty$ with the following property. Suppose that

- $\mathcal{M}$ is a $\varkappa$-solution defined on $(-\infty, 0]$;

- $(x, 0) \in \mathcal{M}_{0}$;

- $l: \mathcal{M}_{<0} \rightarrow \mathbb{R}$ is the $l$-function on $\mathcal{M}$ with spacetime basepoint $(x, 0)$.

Then, there is a partition $\mathcal{P}=\left\{-\infty<T_{0}<\ldots<T_{i}=-1\right\}$ of $(-\infty,-1]$, with $i \leqslant 2\left|\mathcal{V}_{\text {sol }}\right|$, such that if $I_{j}=\left[T_{j-1}, T_{j}\right]$ for $j>0$ and $I_{0}=\left(-\infty, T_{0}\right]$, then the following hold:

(1) if $j$ is odd, then $T_{j-1} / T_{j}<\varrho$;

(2) if $j$ is even, then

(2a) for every $(y, t) \in \mathcal{M}_{I_{j}}$, with $l(y, t)<C$, the rescaled triple $(\widehat{\mathcal{M}}(-t),(y, t), l)$ is $\hat{\varepsilon}$-close to a triple $\left(\mathcal{M}^{\infty},\left(y_{\infty},-1\right), l_{\infty}\right)$, where $\mathcal{M}^{\infty}$ is a gradient shrinking soliton with potential function $l_{\infty}$, and $\widetilde{V}(t)$ is $\hat{\varepsilon}$-close to the reduced volume of $\mathcal{M}^{\infty}$ (see $§ \mathrm{~A} .2$ for the definition of $\hat{\varepsilon}$-closeness of triples);

(2b) $\left|\widetilde{V}\left(I_{j}\right) \cap \mathcal{V}_{\text {sol }}\right|=1$ if $j>0$ and $\left|\widetilde{V}\left(I_{j}\right) \cap \mathcal{V}_{\text {sol }}\right|=0$ if $j=0$;

(2c) $\widetilde{V}(t)$ tends to an element of $\mathcal{V}_{\text {sol }}$ as $t \rightarrow-\infty$.

We begin with a lemma.

Lemma B.2. For all $\varepsilon_{1}>0$ and $C<\infty$ there exist $\mu=\mu\left(\varepsilon_{1}, C\right)<\infty$ and $\theta\left(\varepsilon_{1}, C\right)>0$ with the following property.

Let $\mathcal{M}$ be a $\varkappa$-solution defined on $(-\infty, 0]$, let $(x, 0) \in \mathcal{M}_{0}$ be a basepoint and let $l: \mathcal{M}_{<0} \rightarrow \mathbb{R}$ be the corresponding l-function. Suppose that

- $T^{\prime}<T<0$;

- $T^{\prime} / T \geqslant \mu$;

- $\tilde{V}(T) \leqslant \widetilde{V}\left(T^{\prime}\right)+\theta$;

- $(y, T) \in \mathcal{M}_{T}$ is a point where $l(y, T)<C$. 
Then, the rescaled triple $(\widehat{\mathcal{M}}(-T),(y,-1), l)$ is $\varepsilon_{1}$-close to a triple

$$
\left(\mathcal{M}^{\infty},\left(y_{\infty},-1\right), l_{\infty}\right)
$$

where $\mathcal{M}^{\infty}$ is a gradient shrinking soliton with potential function $l_{\infty}$, and $\widetilde{V}(T)$ is $\varepsilon_{1}$ close to the reduced volume of $\mathcal{M}^{\infty}$.

Proof. Suppose the lemma were false. Then, for some $\varepsilon_{1}>0$ and $C<\infty$ there would be sequences $\left\{\mathcal{M}^{j}\right\}_{j=1}^{\infty},\left\{T_{j}^{\prime}\right\}_{j=1}^{\infty},\left\{T_{j}\right\}_{j=1}^{\infty}$ and $\left\{y_{j}\right\}_{j=1}^{\infty}$ with $T_{j}^{\prime}<T_{j}<0,\left(y_{j}, T_{j}\right) \in \mathcal{M}^{j}$, $\lim _{j \rightarrow \infty} T_{j}^{\prime} / T_{j}=\infty$ and $\lim _{j \rightarrow \infty}\left|\widetilde{V}\left(T_{j}\right)-\widetilde{V}\left(T_{j}^{\prime}\right)\right|=0$, but for each $j$ the rescaled triple $\left(\widehat{M}^{j}\left(-T_{j}\right),\left(y_{j},-1\right), l_{j}\right)$ does not satisfy the conclusion of the lemma. Proposition 6.4 implies that, after passing to a subsequence, we have convergence of triples to a $\varkappa$ solution $\left(\mathcal{M}^{\infty},\left(y_{\infty},-1\right), l_{\infty}\right)$. From the assumed properties of $\left\{\mathcal{M}^{j}\right\}_{j=1}^{\infty}$, the reduced volume $\widetilde{V}_{\infty}(t)$ of $\mathcal{M}^{\infty}$ will be constant for $t \leqslant-1$. Hence, $\mathcal{M}^{\infty}$ is a gradient shrinking soliton, which gives a contradiction.

Proof of Proposition B.1. From the monotonicity of the reduced volume $\widetilde{V}(t)$, the existence of the asymptotic soliton and the fact that $\lim _{t \rightarrow 0} \tilde{V}(t)=\widetilde{V}_{\mathbb{R}^{3}}$ (the reduced volume of flat $\mathbb{R}^{3}$ as a gradient shrinking soliton), it follows that, for all $t \in(-\infty, 0)$, we have $\widetilde{V}(t) \in\left[\widetilde{V}_{1}, \widetilde{V}_{\mathbb{R}^{3}}\right]$.

Let $\mu_{1}=\mu(\hat{\varepsilon}, C)$ and $\theta_{1}=\theta(\hat{\varepsilon}, C)$ be as in Lemma B.2. Choose $\theta_{1}^{\prime}<\min \left(\frac{1}{2} \theta_{1}, \widetilde{V}_{1}\right)$ small enough so that the intervals $\left\{\left[\widetilde{V}_{j}-\theta_{1}^{\prime}, \widetilde{V}_{j}+\theta_{1}^{\prime}\right]\right\}_{1 \leqslant j \leqslant K}$ are disjoint. Put

$$
\tilde{J}_{j}=\left[\widetilde{V}_{j}+\theta_{1}^{\prime}, \tilde{V}_{j+1}-\theta_{1}^{\prime}\right] \text { for } 1 \leqslant j<K,
$$

and $\tilde{J}_{K}=\left[\widetilde{V}_{K}+\theta_{1}^{\prime}, \widetilde{V}_{\mathbb{R}^{3}}\right]$.

Putting $J_{j}=\widetilde{V}^{-1}\left(\tilde{J}_{j}\right) \cap(-\infty,-1]$ for $1 \leqslant j \leqslant K$, we obtain a collection $\left\{J_{j}\right\}_{1 \leqslant j \leqslant K}$ of at most $K$ intervals in $(-\infty,-1]$.

We know that, for each $t<0$, there is a point $y_{t} \in \mathcal{M}_{t}$ with $l\left(y_{t}, t\right) \leqslant \frac{3}{2}$. Let

$$
\mu_{2}=\mu\left(\theta_{1}^{\prime}, 2\right) \quad \text { and } \quad \theta_{2}=\theta\left(\theta_{1}^{\prime}, 2\right)
$$

be as in Lemma B.2. Note that, for every $1 \leqslant j \leqslant K$, $\operatorname{since} \operatorname{dist}\left(\widetilde{V}(t), \mathcal{V}_{\text {sol }}\right) \geqslant \theta_{1}^{\prime}$ for all $t \in J_{j}$, the contrapositive of Lemma B.2 implies that, for any $T^{\prime}, T \in J_{j}$ with $\widetilde{V}(T) \leqslant \widetilde{V}\left(T^{\prime}\right)+\theta_{2}$, we have $T^{\prime} / T<\mu_{2}$. Iterating this $1+\left\lfloor\theta_{2}^{-1} \operatorname{length}\left(J_{j}\right)\right\rfloor$ times gives a bound

$$
\frac{\inf J_{j}}{\sup J_{j}}<\mu_{3}=\mu_{3}(\hat{\varepsilon}, C) \text {. }
$$

The complement of $\bigcup_{j} J_{j}$ in $(-\infty,-1]$ is a union of open intervals. Let $I=\left(t_{-}, t_{+}\right)$ be one such interval. Then, $\left.\widetilde{V}\right|_{I}$ takes values in an interval of length at most $2 \theta_{1}^{\prime} \leqslant \theta_{1}$. 
Therefore, by Lemma B.2, if $t \in I$ and $t \geqslant t_{-} / \mu_{1}$, then for all $y \in \mathcal{M}_{t}$ with $l(y, t)<C$, conclusion (2a) of Proposition B.1 holds.

Thus, we may obtain the desired partition of $(-\infty,-1]$ by enlarging each nonempty interval $J_{j}=\left[t_{j-1}, t_{j}\right]$ to $J_{j}^{\prime}=\left[t_{j-1}, t_{j} / \mu_{1}\right]$, forming the union $\bigcup_{j} J_{j}^{\prime}$, and taking the associated partition of $(-\infty,-1]$. Conclusion $(2 \mathrm{~b})$ of the proposition follows from the construction. Conclusion (2c) of the proposition follows from the finiteness of $\mathcal{V}_{\text {sol }}$, the monotonicity of the reduced volume and the existence of the asymptotic soliton.

Corollary B.3. With the notation of Proposition B.1, depending on the topology of $\mathcal{M}_{0}$, the sequence $\left\{\mathcal{M}^{2 j}\right\}_{0 \leqslant 2 j \leqslant i}$ of gradient shrinking solitons must necessarily be one of the following:

(1) $\mathcal{M}_{0}$ is diffeomorphic to a spherical space form other than $S^{3}$ or $S^{3} / \mathbb{Z}_{2}$ :

$\{$ Sphere $/ \Gamma\}$

(2) $\mathcal{M}_{0}$ is diffeomorphic to the cylinder:

$$
\{\mathrm{Cyl}\}
$$

(3) $\mathcal{M}_{0}$ is diffeomorphic to the $\mathbb{Z}_{2}$-quotient of the cylinder:

$$
\left\{\mathrm{Cyl} / \mathbb{Z}_{2}, \mathrm{Cyl}\right\}, \quad\left\{\mathrm{Cyl} / \mathbb{Z}_{2}\right\}, \quad\{\mathrm{Cyl}\}
$$

(4) $\mathcal{M}_{0}$ is diffeomorphic to $S^{3}$ :

$\{\mathrm{Cyl}$, Sphere $\}, \quad\{$ Sphere $\}, \quad\{\mathrm{Cyl}\}$

(5) $\mathcal{M}_{0}$ is diffeomorphic to $S^{3} / \mathbb{Z}_{2}$ :

$\left\{\mathrm{Cyl} / \mathbb{Z}_{2}\right.$, Sphere $\left./ \mathbb{Z}_{2}\right\}, \quad\left\{\right.$ Sphere $\left./ \mathbb{Z}_{2}\right\}, \quad\left\{\mathrm{Cyl} / \mathbb{Z}_{2}\right\}, \quad\left\{\mathrm{Cyl} / \mathbb{Z}_{2}, \mathrm{Cyl}\right\}, \quad\{\mathrm{Cyl}\}$.

Proof. The relevant gradient shrinking solitons are Cyl $/ \mathbb{Z}_{2}$, Sphere $/ \mathbb{Z}_{2}$, Cyl and Sphere. From Lemma A.2, their reduced volumes are 16.39, 17.62, 32.78 and 35.24, respectively. The corollary follows from the fact that the reduced volumes of the $\mathcal{M}^{2 j}$ 's form a strictly increasing sequence, the local stability of quotients of Sphere, and topological restrictions.

Remark B.4. From explicit calculations, one can rule out the $\{\mathrm{Cyl}\}$ possibility in case (3) of Corollary B.3. We expect that one can also rule out the $\{\mathrm{Cyl}\}$ possibility in case (5). 


\section{Appendix C. Non-negative isotropic curvature in four dimensions}

In this appendix we extend the results of the paper to 4-dimensional Ricci flow with non-negative isotropic curvature, under an assumption of no incompressible embedded spherical space forms. The basic results about such flows are due to Hamilton [35].

Let $(M, g)$ be a closed oriented 4-manifold with non-negative isotropic curvature. Suppose that, under Ricci flow, the isotropic curvature does not immediately become positive. By [54, Theorem 4.10], one of the following statements holds:

(1) $(M, g)$ is flat;

(2) the universal cover $(\tilde{M}, \tilde{g})$ is an isometric product of metrics on $S^{2}$ and a surface $\Sigma$;

(3) $M$ is biholomorphic to $\mathbb{C} P^{2}$ and $g$ is a Kähler metric with positive first Chern class.

In these three cases, the Ricci flow is well understood. In case (1), it is just a static flow. In cases (2) and (3), the ensuing Ricci flow will be smooth on a time interval $[0, T)$ and non-existent thereafter. Hence, we assume that $(M, g)$ has positive isotropic curvature. This condition is preserved under the Ricci flow.

There is a version of the Hamilton-Ivey inequality for Ricci flow on a 4-manifold with positive isotropic curvature $[35, \S \mathrm{B}]$. It has the implication that any blow-up limit is a $\varkappa$-solution with positive isotropic curvature, and restricted isotropic curvature in the sense of [18, equation (2.4)]. Any such compact solution is diffeomorphic to $S^{4}$. Any such non-compact solution is diffeomorphic to $\mathbb{R}^{4}$, or is an isometric product $\mathbb{R} \times Z$, where $Z$ is diffeomorphic to a spherical space form.

Using [56], we have the following extension of Lemma A.2.

Lemma C.1. Let $\mathcal{M}$ be a 4-dimensional gradient shrinking soliton that is a $\varkappa$ solution, has positive isotropic curvature and restricted isotropic curvature in the terminology of $[18$, equation (2.4)], and blows up as $t \rightarrow 0$. Given $t<0$ and a point $(y, t) \in \mathcal{M}$, let $l_{y, t} \in C^{\infty}\left(\mathcal{M}_{<t}\right)$ be the $l$-function on $\mathcal{M}$ constructed using $\mathcal{L}$-geodesics going backward in time from $(y, t)$. Then, there is a function $l_{\infty} \in C^{\infty}(\mathcal{M})$ such that the limit

$$
\lim _{t \rightarrow 0^{-}} l_{y, t}=l_{\infty}
$$

exists, independent of $y$, with continuous convergence on compact subsets on $\mathcal{M}$. Define $\widetilde{V}_{\infty}:(-\infty, 0) \rightarrow(0, \infty)$ as in $(6.5)$. Then, one of the following statements holds:

(1) $\mathcal{M}$ is isometric to the shrinking round cylinder solution on $\left(S^{3} / \Gamma\right) \times \mathbb{R}$, where $\Gamma \subset \mathrm{SO}(4)$, with

$$
R(x, t)=\frac{3}{2}(-t)^{-1}, \quad l_{\infty}((x, z), t)=\frac{3}{2}+\frac{z^{2}}{-4 t} \quad \text { and } \quad \tilde{V}_{\infty}(t)=\frac{32 \pi^{5 / 2} e^{-3 / 2}}{|\Gamma|}
$$


(2) $\mathcal{M}$ is a shrinking round $S^{4}$, with

$$
R(x, t)=2(-t)^{-1}, \quad l_{\infty}(x, t)=2 \quad \text { and } \quad \widetilde{V}_{\infty}(t)=\frac{96 \pi^{2}}{e^{2}} .
$$

Let $(M, g)$ be a compact 4-manifold with positive isotropic curvature and no embedded $\pi_{1}$-injective spherical space forms. The construction of Ricci flow with surgery, starting from $(M, g)$, was initiated by Hamilton [35]. There was a problem with [35] because no canonical neighborhood result was available at that time. The construction was revisited by Chen-Zhu [18], based on Perelman's work in the 3-dimensional case [57], [58] and a preprint version of [44]. The result of Lemma C.1, which follows from [56], was not available when [18] was written. With the incorporation of Lemma C.1, the construction of the Ricci flow with surgery is strictly analogous to Perelman's work.

The results of the present paper now extend to the setting of 4-dimensional singular Ricci flows with positive isotropic curvature, again under the assumption that the initial time slice does not have any embedded $\pi_{1}$-injective spherical space forms.

Returning to Ricci flow with surgery, suppose that there are such embedded spherical space forms in the initial time slice. There is still a well-defined Ricci flow with surgery, but the time slices may be orbifolds with isolated singularities [17]. It should be possible to extend the results of the present paper to the orbifold setting, using [45], but we do not address the subject here.

\section{References}

[1] Allard, W. K., On the first variation of a varifold. Ann. of Math., 95 (1972), 417-491.

[2] Almgren, F. J., JR., Some interior regularity theorems for minimal surfaces and an extension of Bernstein's theorem. Ann. of Math., 84 (1966), 277-292.

[3] Angenent, S. B., Caputo, M. C. \& Knopf, D., Minimally invasive surgery for Ricci flow singularities. J. Reine Angew. Math., 672 (2012), 39-87.

[4] Angenent, S. B. \& Knopf, D., Precise asymptotics of the Ricci flow neckpinch. Comm. Anal. Geom., 15 (2007), 773-844.

[5] Ballmann, W., Lectures on Spaces of Nonpositive Curvature. DMV Seminar, 25. Birkhäuser, Basel, 1995.

[6] Bamler, R.H. \& Kleiner, B., Ricci flow and diffeomorphism groups of 3-manifolds. Preprint, 2017. arXiv:1712.06197 [math.DG].

[7] — Uniqueness and stability of Ricci flow through singularities. Preprint, 2017. arXiv: 1709.04122 [math.DG].

[8] Bethuel, F., On the singular set of stationary harmonic maps. Manuscripta Math., 78 (1993), 417-443.

[9] Brakke, K. A., The Motion of a Surface by its Mean Curvature. Mathematical Notes, 20. Princeton Univ. Press, Princeton, NJ, 1978.

[10] Brendle, S. \& Huisken, G., Mean curvature flow with surgery of mean convex surfaces in $\mathbb{R}^{3}$. Invent. Math., 203 (2016), 615-654. 
[11] Cheeger, J., Gromov, M. \& Taylor, M., Finite propagation speed, kernel estimates for functions of the Laplace operator, and the geometry of complete Riemannian manifolds. J. Differential Geom., 17 (1982), 15-53.

[12] Chen, X. X., Tian, G. \& Zhang, Z., On the weak Kähler-Ricci flow. Trans. Amer. Math. Soc., 363 (2011), 2849-2863.

[13] Chen, X. X. \& WAng, Y., Bessel functions, heat kernel and the conical Kähler-Ricci flow. J. Funct. Anal., 269 (2015), 551-632.

[14] — On the long time behaviour of the conical Kähler-Ricci flows. To appear in J. Reine Angew. Math.

[15] Chen, Y. G., Giga, Y. \& Goto, S., Uniqueness and existence of viscosity solutions of generalized mean curvature flow equations. J. Differential Geom., 33 (1991), 749-786.

[16] Chen, Y. M. \& Struwe, M., Existence and partial regularity results for the heat flow for harmonic maps. Math. Z., 201 (1989), 83-103.

[17] Chen, B.-L., TAng, S.-H. \& Zhu, X.-P., Complete classification of compact fourmanifolds with positive isotropic curvature. J. Differential Geom., 91 (2012), 41-80.

[18] Chen, B.-L. \& Zhu, X.-P., Ricci flow with surgery on four-manifolds with positive isotropic curvature. J. Differential Geom., 74 (2006), 177-264.

[19] Chow, B., On the entropy estimate for the Ricci flow on compact 2-orbifolds. J. Differential Geom., 33 (1991), 597-600.

[20] Chow, B., Chu, S.-C., Glickenstein, D., Guenther, C., Isenberg, J., Ivey, T., Knopf, D., Lu, P., Luo, F. \& Ni, L., The Ricci Flow: Techniques and Applications. Part I. Mathematical Surveys and Monographs, 135. Amer. Math. Soc., Providence, RI, 2007.

[21] Chow, B., Lu, P. \& Ni, L., Hamilton's Ricci Flow. Graduate Studies in Mathematics, 77. Amer. Math. Soc., Providence, RI, 2006.

[22] Chow, B. \& Wu, L. F., The Ricci flow on compact 2-orbifolds with curvature negative somewhere. Comm. Pure Appl. Math., 44 (1991), 275-286.

[23] De Giorgi, E., Frontiere Orientate di Misura Minima. Seminario di Matematica della Scuola Normale Superiore di Pisa. Editrice Tecnico Scientifica, Pisa, 1961.

[24] Enders, J., Reduced distance based at singular time in the ricci flow. Preprint, 2007. arXiv:0711.0558 [math.DG],.

[25] Evans, L. C. \& Spruck, J., Motion of level sets by mean curvature. I. J. Differential Geom., 33 (1991), 635-681.

[26] — Motion of level sets by mean curvature. IV. J. Geom. Anal., 5 (1995), 77-114.

[27] Eyssidieux, P., Guedu, V. \& Zeriahi, A., Weak solutions to degenerate complex MongeAmpère flows II. Adv. Math., 293 (2016), 37-80.

[28] Federer, H., The singular sets of area minimizing rectifiable currents with codimension one and of area minimizing flat chains modulo two with arbitrary codimension. Bull. Amer. Math. Soc., 76 (1970), 767-771.

[29] Federer, H. \& Fleming, W. H., Normal and integral currents. Ann. of Math., 72 (1960), 458-520.

[30] Feldman, M., Ilmanen, T. \& Knopf, D., Rotationally symmetric shrinking and expanding gradient Kähler-Ricci solitons. J. Differential Geom., 65 (2003), 169-209.

[31] Giaquinta, M. \& Giusti, E., The singular set of the minima of certain quadratic functionals. Ann. Scuola Norm. Sup. Pisa Cl. Sci., 11 (1984), 45-55.

[32] Giesen, G. \& Topping, P. M., Existence of Ricci flows of incomplete surfaces. Comm. Partial Differential Equations, 36 (2011), 1860-1880.

[33] — Ricci flows with unbounded curvature. Math. Z., 273 (2013), 449-460. 
[34] Hamilton, R. S., A compactness property for solutions of the Ricci flow. Amer. J. Math., 117 (1995), 545-572.

[35] — Four-manifolds with positive isotropic curvature. Comm. Anal. Geom., 5 (1997), 1-92.

[36] - Three-orbifolds with positive Ricci curvature, in Collected Papers on Ricci Flow, Series in Geometry and Topology, 37, pp. 521-524. Int. Press, Somerville, MA, 2003.

[37] Haslhofer, R. \& Kleiner, B., Mean curvature flow with surgery. Duke Math. J., 166 (2017), 1591-1626.

[38] Haslhofer, R. \& Naber, A., Weak solutions for the Ricci flow I. To appear in J. Eur. Math. Soc. (JEMS).

[39] Head, J., On the mean curvature evolution of two-convex hypersurfaces. J. Differential Geom., 94 (2013), 241-266.

[40] HÉlein, F., Regularity of weakly harmonic maps from a surface into a manifold with symmetries. Manuscripta Math., 70 (1991), 203-218.

[41] Hörmander, L., The Analysis of Linear Partial Differential Operators. I. Classics in Mathematics. Springer, Berlin-Heidelberg, 2003.

[42] Huisken, G., Asymptotic behavior for singularities of the mean curvature flow. J. Differential Geom., 31 (1990), 285-299.

[43] Ilmanen, T., Elliptic regularization and partial regularity for motion by mean curvature. Mem. Amer. Math. Soc., 108 (1994), 90 pp.

[44] Kleiner, B. \& LotT, J., Notes on Perelman's papers. Geom. Topol., 12 (2008), 2587-2855.

[45] - Geometrization of three-dimensional orbifolds via Ricci flow. Astérisque, 365 (2014), 101-177.

[46] Косн, H. \& Lamm, T., Geometric flows with rough initial data. Asian J. Math., 16 (2012), 209-235.

[47] Lauer, J., Convergence of mean curvature flows with surgery. Comm. Anal. Geom., 21 (2013), 355-363.

[48] Lin, F.-H., Gradient estimates and blow-up analysis for stationary harmonic maps. Ann. of Math., 149 (1999), 785-829.

[49] Lin, F.-H. \& WANG, C.-Y., Harmonic and quasi-harmonic spheres. III. Rectifiability of the parabolic defect measure and generalized varifold flows. Ann. Inst. H. Poincaré Anal. Non Linéaire, 19 (2002), 209-259.

[50] Liu, J. \& Zhang, X., Conical Kähler-Ricci flows on Fano manifolds. Adv. Math., 307 (2017), 1324-1371.

[51] Lu, P., A compactness property for solutions of the Ricci flow on orbifolds. Amer. J. Math., 123 (2001), 1103-1134.

[52] Mazzeo, R., Rubinstein, Y. A. \& Sesum, N., Ricci flow on surfaces with conic singularities. Anal. PDE, 8 (2015), 839-882.

[53] Metzger, J. \& Schulze, F., No mass drop for mean curvature flow of mean convex hypersurfaces. Duke Math. J., 142 (2008), 283-312.

[54] Micallef, M. J. \& WANG, M. Y., Metrics with nonnegative isotropic curvature. Duke Math. J., 72 (1993), 649-672.

[55] Morrey, C. B., JR., Multiple Integrals in the Calculus of Variations. Die Grundlehren der mathematischen Wissenschaften, 130. Springer, New York, 1966.

[56] NABER, A., Noncompact shrinking four solitons with nonnegative curvature. J. Reine Angew. Math., 645 (2010), 125-153.

[57] Perelman, G., The entropy formula for the Ricci flow and its geometric applications. Preprint, 2002. arXiv:math/0211159 [math.DG].

[58] — Ricci flow with surgery on three-manifolds. Preprint, 2003. arXiv:math/0303109 [math.DG]. 
[59] Phong, D. H., Song, J., Sturm, J. \& Wang, X., The Ricci flow on the sphere with marked points. Preprint, 2014. arXiv:1407.1118 [math.DG],.

[60] Reifenberg, E. R., Solution of the Plateau Problem for $m$-dimensional surfaces of varying topological type. Acta Math., 104 (1960), 1-92.

[61] — On the analyticity of minimal surfaces. Ann. of Math., 80 (1964), 15-21.

[62] Schnürer, O.C., Schulze, F. \& Simon, M., Stability of Euclidean space under Ricci flow. Comm. Anal. Geom., 16 (2008), 127-158.

[63] Schoen, R. \& Uhlenbeck, K., A regularity theory for harmonic maps. J. Differential Geom., 17 (1982), 307-335. Correction in J. Differential Geom., 18 (1983), 329.

[64] - Regularity of minimizing harmonic maps into the sphere. Invent. Math., 78 (1984), 89-100.

[65] Simon, L., Cylindrical tangent cones and the singular set of minimal submanifolds. J. Differential Geom., 38 (1993), 585-652.

[66] - Rectifiability of the singular set of energy minimizing maps. Calc. Var. Partial Differential Equations, 3 (1995), 1-65.

[67] Simon, M., Deformation of $C^{0}$ Riemannian metrics in the direction of their Ricci curvature. Comm. Anal. Geom., 10 (2002), 1033-1074.

[68] — Ricci flow of almost non-negatively curved three manifolds. J. Reine Angew. Math., 630 (2009), 177-217.

[69] — Ricci flow of non-collapsed three manifolds whose Ricci curvature is bounded from below. J. Reine Angew. Math., 662 (2012), 59-94.

[70] Simons, J., Minimal varieties in riemannian manifolds. Ann. of Math., 88 (1968), 62-105.

[71] Song, J. \& Tian, G., The Kähler-Ricci flow through singularities. Invent. Math., 207 (2017), 519-595.

[72] Struwe, M., On the evolution of harmonic maps in higher dimensions. J. Differential Geom., 28 (1988), 485-502.

[73] Topping, P. M., Lectures on the Ricci Flow. London Mathematical Society Lecture Note Series, 325. Cambridge Univ. Press, Cambridge, 2006.

[74] - Ricci flow compactness via pseudolocality, and flows with incomplete initial metrics. $J$. Eur. Math. Soc. (JEMS), 12 (2010), 1429-1451.

[75] - Ricci flow compactness via pseudolocality, and flows with incomplete initial metrics. $J$. Eur. Math. Soc. (JEMS), 12 (2010), 1429-1451.

[76] - Uniqueness and nonuniqueness for Ricci flow on surfaces: reverse cusp singularities. Int. Math. Res. Not., (2012), 2356-2376.

[77] Wang, Y., Smooth approximations of the conical Kähler-Ricci flows. Math. Ann., 365 (2016), 835-856.

[78] White, B., The size of the singular set in mean curvature flow of mean-convex sets. $J$. Amer. Math. Soc., 13 (2000), 665-695.

[79] - The nature of singularities in mean curvature flow of mean-convex sets. J. Amer. Math. Soc., 16 (2003), 123-138.

[80] Wu, L. F., The Ricci flow on 2-orbifolds with positive curvature. J. Differential Geom., 33 (1991), 575-596.

[81] YE, R., On the $l$-function and the reduced volume of Perelman. I. Trans. Amer. Math. Soc., 360 (2008), 507-531.

[82] - On the $l$-function and the reduced volume of Perelman. II. Trans. Amer. Math. Soc., 360 (2008), 533-544. 
[83] Yın, H., Ricci flow on surfaces with conical singularities. J. Geom. Anal., 20 (2010), 970-995.

[84] — Ricci flow on surfaces with conical singularities II. Preprint, 2013. arXiv:1305.4355 [math.DG].

Bruce Kleiner

Courant Institute of Mathematical Sciences 251 Mercer St.

New York, NY 10012

U.S.A.

bkleiner@cims.nyu.edu

Received November 16, 2015
John LOTT

Department of Mathematics

University of California at Berkeley

Berkeley, CA 94720

U.S.A.

lott@berkeley .edu 